Florida International University FIU Digital Commons

\title{
A computer based approach to improve the dietary and physical activity patterns of a diverse group of adolescents
}

\author{
Krista Casazza \\ Florida International University
}

DOI: $10.25148 /$ etd.FI14052594

Follow this and additional works at: https://digitalcommons.fiu.edu/etd

Part of the Dietetics and Clinical Nutrition Commons

\section{Recommended Citation}

Casazza, Krista, "A computer based approach to improve the dietary and physical activity patterns of a diverse group of adolescents" (2006). FIU Electronic Theses and Dissertations. 2022.

https://digitalcommons.fiu.edu/etd/2022 
Miami, Florida

A COMPUTER BASED APPROACH TO IMPROVE THE DIETARY AND PHYSICAL ACTIVITY PATTERNS OF A DIVERSE GROUP OF ADOLESCENTS

A dissertation submitted in partial fulfillment of the

requirements for the degree of

DOCTOR OF PHILOSOPHY

in

DIETETICS AND NUTRITION

by

Krista Casazza

2006 
To: Interim Executive Dean Ray Thomlison

College of Health and Urban Affairs

This dissertation, written by Krista Casazza, and entitled A Computer Based Approach to Improve the Dietary and Physical Activity Patterns of a Diverse Group of Adolescents, having been approved in respect to style and intellectual content, is referred to you for judgment.

We have read this dissertation and recommend that it be approved.

Fatma G. Huffman

Marianna K. Baum

Paulette Johnson

Michele Ciccazzo, Major Professor

Date of Defense: March 31, 2006

The dissertation of Krista Casazza is approved.

Interim Executive Dean Ray Thomlison

College of Health and Urban Affairs

Interim Dean Stephan L. Mintz

University Graduate School

Florida International University, 2006 


\section{DEDICATION}

To my best friend, for without the love, support, and patience, none of this would have been possible. 


\section{ACKNOWLEDGMENTS}

I would like to give a special thanks to the following:

Members of my Committee Dr. Michele Ciccazzo, Major professor

Dr. Fatma Huffman

Dr. Marianna Baum

Dr. Paulette Johnson

Florida International University

School Board of Broward County

High School Principals at Participating Schools

Data Collection Assistants Lucretia Potts

Sheryl Barrett

Lynn Kunins

Stacy Samlal

School Teachers at Participating Schools

Students who participated in the study

This study was supported financially by the following:

Florence Bayuk Graduate Fellowship in Health from the Florence Bayuk Foundation

Dissertation Year Fellowship, FIU University Graduate School Dissertation Fellowship 
ABSTRACT OF THE DISSERTATION

A COMPUTER BASED APPROACH TO IMPROVE THE DIETARY AND

PHYSICAL ACTIVITY PATTERNS OF A DIVERSE GROUP OF ADOLESCENTS

by

Krista Casazza

Florida International University, 2006

Miami, Florida

Professor Michele Ciccazzo, Major Professor

Diet and physical activity patterns have been implicated as major factors in the increasing prevalence of childhood and adolescent obesity. It is estimated that between 16 and 33 percent of children and adolescents in the United States are overweight (CDC, 2000). Moreover, the CDC estimates that less than $50 \%$ of adolescents are physically active on a regular basis (CDC, 2003). Interventions must be focused to modify these behaviors. Facilitating the understanding of proper nutrition and need for physical activity among adolescents is the first step in preventing overweight and obesity and delaying the development of chronic diseases later in life (Dwyer, 2000). The purpose of this study was to compare the outcomes of students receiving one of two forms of education (both emphasizing diet and physical activity), to determine whether a computer based intervention (CBI) program using an interactive, animated CD-ROM would elicit a greater behavior change in comparison to a traditional didactic intervention (TDI) program. A convenience sample of 254 high school students aged 14-19 participated in the 6-month program. A pre-test post-test design was used, with follow-up measures taken at three months post-intervention. 
No change was noted in total fat, saturated fat, fruit/vegetables, or fiber intake for any of the groups. There was also no change in perceived self-efficacy or perceived social support. Results did, however, indicate an increase in nutrition knowledge for both intervention groups $(\mathrm{p}<0.001)$. In addition, the CBI group demonstrated more positive and sustained behavior changes throughout the course of the study. These changes included a decrease in BMI ( $\mathrm{p}_{\text {pre/ post }}<0.001, \mathrm{p}_{\text {post }}$ follow-up $\left.<0.001\right)$, number of meals skipped $\left(\mathrm{p}_{\text {pre/post }}<0.001\right)$, and soda consumption $\left(\mathrm{p}_{\text {pre/post }}=0.003, \mathrm{p}_{\text {post/follow-up }}=0.03\right)$ and an increase in nutrition knowledge $\left(\mathrm{p}_{\text {pre/post }}<0.001, \mathrm{p}_{\text {pre/follow-up }}<0.001\right)$, physical activity $\left(\mathrm{p}_{\text {pre/post }}<0.05\right.$, $\left.\mathrm{p}_{\text {pre/follow-up }}<0.01\right)$, frequency of label reading $\left(\mathrm{p}_{\text {pre/follow-up }}<0.01\right)$ and in dairy consumption $\left(\mathrm{p}_{\text {pre/post }}=0.03\right)$. The TDI group did show positive gains in some areas post intervention, however a return to baseline behavior was shown at follow-up. Findings of this study suggest that compared to traditional didactic teaching, computer-based nutrition and health education has greater potential to elicit change in knowledge and behavior as well as promote maintenance of the behavior change over time. 
I. INTRODUCTION

II. REVIEW OF THE LITERATURE 4

Population $\quad 4$

Diet Quality 5

Overweight, Obesity, and Other Risk Factors 8

Type 2 Diabetes $\quad 11$

Gender Differences 14

Racial Differences $\quad 15$

Skipping Meals 16

Socioeconomic Status 17

$\begin{array}{ll}\text { Physical Activity } & 19\end{array}$

Interventions $\quad 23$

Nutrition Education $\quad 23$

Barriers to Nutrition Education $\quad 25$

Computer-Based Learning 27

Applications

Using the Practical Approach 31

Empowerment $\quad 32$

Self-Efficacy 33

Social Support $\quad 35$

Behavioral Focus $\quad 36$

Purpose $\quad 37$

Research Questions $\quad 37$

III. RESEARCH METHODOLOGY 39

Development of Behaviorally Focused Intervention Program 39

Preliminary Qualitative Study Used in Tailoring $\quad 39$

Intervention Approach $\quad 42$

Preliminary Quantitative Study $\quad 45$

Research Study $\quad 48$

Recruitment of Schools $\quad 50$

Recruitment of Students $\quad 51$

Screening 53

Eligibility and Inclusion/Exclusion Criteria $\quad 53$

Study Design $\quad 54$

Baseline Measures $\quad 54$

$\begin{array}{ll}\text { Instruments } & 56\end{array}$

Anthropometric $\quad 56$

Height and Weight Measure 
Dietary Assessment

24-Hour Recall 57

Food Frequency Questionnaire $\quad 59$

Nutrition Knowledge

Nutrition Knowledge Questionnaire $\quad 61$

Physical Activity Assessment

Physical Activity Questionnaire for Adolescents (PAQ-a) 62

Self-Efficacy

Dietary and Exercise Confidence Survey 63

Social Support

Social Support Survey $\quad 65$

Incentives

66

Confidentiality of Data 66

$\begin{array}{ll}\text { Statistical Analysis } & 67\end{array}$

Analysis of Questionnaire Responses $\quad 69$

IV. RESULTS

Sample Characteristics $\quad 71$

$\begin{array}{ll}\text { Outcome Measures } & 71\end{array}$

Hypothesis $1 \quad 71$

Hypothesis 2 74

Hypothesis $3 \quad 76$

Hypothesis $4 \quad 88$

Hypothesis 5

Hypothesis $6 \quad 90$

Hypothesis $7 \quad 90$

Other Dietary Findings $\quad 94$

Additional Findings $\quad 96$

V. DISCUSSION AND CONCLUSIONS 97

Discussion of Hypothesis $\quad 97$

Nutrition Knowledge $\quad 98$

Dietary Intake $\quad 99$

Physical Activity 107

BMI 109

Other Dietary Behaviors $\quad 110$

Label Reading $\quad 110$

Meal Skipping 110

Psychosocial Factors $\quad 111$

Self-Efficacy $\quad 111$

Social Support 113

$\begin{array}{ll}\text { Limitations of the Study } & 114\end{array}$ 
Conclusions

115

Applications

116

Future Studies

REFERENCES

119

APPENDICES

134

VITA

220 


\section{LIST OF TABLES}

TABLE

1. Sociodemographic Data from School Used in Preliminary Studies

2. Study Schools Ethnic, Gender, FCAT and Free Lunch Breakdown (2003-2004) 52

3. Demographics for Study Participants

4. The Effect of a Computer-based and a Didactic Intervention on Nutrition

Knowledge Scores (\% correct)

5. The Effect of a Computer-based and a Didactic Intervention on Nutrition

Knowledge (\# correct) per Category

6. The Effect of a Computer-based and a Didactic Intervention on Average

BMI Over Time

6a. The Effect of a Computer-based and a Didactic Intervention on the Change

75 in BMI Categories Over Time

7. The Effect of a Computer-based and a Didactic Intervention on Average

Kilocalorories Consumed per Day Based on Two Non-consecutive 24-hour Recalls

8. The Effect of a Computer-based and a Didactic Intervention on Average Fat

(g) Intake per Day as Measured by Two Non-consecutive 24-hour Recalls

9. The Effect of a Computer-based and a Didactic Intervention on Average Fat

Day as Measured by Food Frequency Questionnaire (FFQ)

10. The Effect of a Computer-based and a Didactic Intervention on Average

Saturated Fat Intake per Day as Measured by Two Non-consecutive

24-hour Recalls

11. The Effect of a Computer-based and a Didactic Intervention on Average

Fiber (g) Intake per Day as Measured by Two Non-consecutive 24-hour Recalls

12. The Effect of a Computer-based and a Didactic Intervention on Average

Fiber (g) Intake per Day as Measured by Food Frequency Questionnaire (FFQ) 
13. The Effect of a Computer-based and a Didactic Intervention on Fruit and

Vegetable Intake (servings/day) as Reported by Two Non-consecutive 24-hour Recalls

14. The Effect of a Computer-based and a Didactic Intervention on Fruit and

Vegetable Intake (servings/day) as Reported by Food Frequency

Questionnaire (FFQ)

15. The Effect of a Computer-based and a Didactic Intervention on Dairy

Intake (servings/day) as Reported by Two Non-consecutive 24-hour Recalls

16. The Effect of a Computer-based and a Didactic Intervention on Average

Protein (g) Intake per Day as Measured by Two Non-consecutive 24-hour Recalls

17. The Effect of a Computer-based and a Didactic Intervention on Average

Carbohydrate (g) Intake per Day as Measured by Two Non-consecutive 24-hour Recalls

18. The Effect of a Computer-based and a Didactic Intervention on average

Cholesterol (mg) Intake per Day as Measured by Two Non-consecutive 24-hour Recalls

19. The Effect of a Computer-based and a Didactic Intervention on Daily a La

Carte Lunch Purchases as Reported by Two Non- consecutive 24-hour Recalls

20. The Effect of a Computer-based and a Didactic Intervention on Average

Amount of Soda (oz) Consumed per Day as Reported by Two

Non-consecutive 24-hour Recalls

21. The Effect of a Computer-based and a Didactic Intervention on Average

Number of Meals Skipped as Reported by Two Non-consecutive 24-hour

Recalls

22. The Effect of a Computer-based and a Didactic Intervention on Self-

Reported Physical Activity as Reported on PAQ

22a. The Effect of a Computer-based and a Didactic Intervention on Percentage

of Students Reporting Daily Moderate to Vigorous Physical Activity

23. The Effect of a Computer-based and a Didactic Intervention on Perceived

Self- Efficacy Scores as Assessed Using Dietary and Exercise

Confidence Survey 
24. The Effect of a Computer-based and a Didactic Intervention on Perceived

25. The Effect of a Computer-based and a Didactic Intervention on Perceived

26. The Effect of a Computer-based and a Didactic Intervention on the Frequency of Label Reading

27. The Effect of a Computer-based and a Didactic Intervention on Micronutrient 95 Intake as Assessed by Two Non-consecutive 24-hour Recalls

28. The Effect of a Computer-based and a Didactic Intervention on the Number 95 of Times Participants Consumed Fast Food as Reported by Two Non-consecutive 24-hour Recalls

29. The Effect of a Computer-based and a Didactic Intervention on Servings of 96 Fries Fries/chips per Day as Reported by Two Non-consecutive 24- hour Recalls 


\section{LIST OF FIGURES}

FIGURE

PAGE

1. Development of Chronic Disease

2. Practical Approach

3. Multi-Pass Method

4. Repeated Measures ANOVA Approach

5. Summary of Methods

70 
LIST OF ACRONYMS

ADA American Diabetes Association

ADA American Dietetic Association

CATCH Child and Adolescent Trial on Cardiovascular Health

CBI Computer Based Intervention

CDC Centers for Disease Control

CHF Congestive Heart Failure

CON Control

CSFII Continuing Survey of Food Intake of Individuals

CVD Cardiovascular Disease

DHHS US Department of Health and Human Services

FCAT Florida Comprehensive Assessment Test

HBM Health Belief Model

HP 2010 Healthy People 2010

HTN Hypertension

NHANES National Health and Nutrition Examination Survey

NIH National Institute of Health

PLAN B Practical Lifestyle Approach to New Behaviors

SES Socioeconomic Status

TDI Traditional Education Intervention 


\section{CHAPTER I}

\section{Introduction}

Adolescence is a period of rapid growth and development associated with increased nutrient needs (Spear, 2002). It is well documented that several chronic diseases, major causes of morbidity and mortality in the US, have their roots in a person's younger years (CDC, 2000). Behavioral patterns established during adolescence are likely to influence long-term health behavior and may have a tremendous impact on lifelong health (Spear, 2002; Hoelscher et al, 2002; Story et al, 2002). While healthy eating habits and lifestyle that begin in childhood or adolescence may decrease the risk of chronic disease, unhealthy dietary habits appear to be associated with increased incidence of chronic disease later in life (Phillipas and Lo, 2005; Lytle, 2002; Cabrinety, 2002). For example, increasingly, type 2 diabetes is being noted in children as young as 12 years old and may be associated with the development of other cardiovascular diseases in adulthood (Fagot-Campagna, 2003; Rami, 2003).

Unhealthy dietary patterns noted among adolescents may be influenced by adolescents' overall lifestyle, including social norms, increased proportion of adolescents that work, and relative inexpensiveness, availability, and popularity of fast foods and convenience foods (Conklin et al, 2005; Nicklas, 2002). Moreover, the Center for Disease Control (CDC) estimates that less than $50 \%$ of adolescents are physically active on a regular basis. As a result of dietary and physical activity patterns, current estimates are that between 16 and 33 percent of children and adolescents in the United States are overweight (Hedley et al, 2004; CDC, 2000). The past four decades have been met with an almost four-fold increase in body mass index that continues to rise each year (Ogden, 
2002). Interventions must be targeted to promote healthy nutrition and physical activity behaviors. Facilitating the understanding of proper nutrition and need for physical activity among adolescents is likely to help prevent health problems such as overweight and obesity and the development of chronic diseases later in life (Dwyer, 2000). Establishing healthy eating and physical activity habits in youth is critical because changing poor eating patterns as one ages is increasingly difficult. Additionally, obesity in childhood is a strong predictor of obesity in adulthood (Galvez et al, 2003).

The American Diabetes Association (ADA) and the CDC have recently described type 2 diabetes as an emerging epidemic among children and adolescents. New incidence of type 2 diabetes among adolescents ranges from $8-45 \%$ depending upon geographic location (Fagot-Campagna, 2003). Moreover, the CDC estimates that nearly one in three children and adolescents will be diagnosed with type 2 diabetes by the year 2025 (CDC, 2003) while other sources report that the prevalence of diabetes worldwide will double by 2030 (Wild et al, 2004). This increase parallels the increase in overweight and obesity. Poor dietary choices, including a diet high in calories and fat and the lack of physical activity noted among children and teens in the past decade, are the two main factors implicated in these recent surges. However, as hypothesized by the Health Belief Model (Glanz et al, 1997), there is a lack of urgency among adolescents associated with their current behaviors and the possible consequences later in life (Story et al, 2002). Without a focus on prevention, it is likely that this population is destined to develop chronic diseases that will require substantial resources in the future (Steinberger and Daniels, 2003). 
Unfortunately, past intervention efforts have been met with little success, as they tend to increase knowledge but do little to elicit a behavior change (Hoelscher et al, 2002; Litchfeld et al, 2000). However, use of technologically enhanced resources, such as a multi-media website, CD-ROM or other computer-assisted programs has been shown to have a greater impact in motivating people to change their diet, in particular their fat and consequently caloric intake and increase physical activity, compared to more traditional intervention methods (Goran and Reynolds, 2005; Vandelanotte et al, 2005: Budman, 2000; Brug et al, 1999; Oenema et al., 2001; Brug et al. 1998; de Vries and Brug, 1999). Using a computer-based approach, participants actually play an active role in the learning process.

The purpose of this research was to determine whether a computer based intervention (CBI) program using an interactive, animated CD-ROM would elicit a greater change in eating behavior and physical activity patterns among high school students than would a traditional didactic intervention (TDI) program. Both education programs had an emphasis on healthy lifestyle habits (diet and physical activity). The purpose was also to determine whether other key variables were differentially affected by the two intervention methods, i.e. nutrition knowledge, body mass index (BMI), self-efficacy in making lifestyle changes, and perceived social support. 


\section{CHAPTER II \\ Review of the Literature}

\section{Overview of the Problem}

\section{Population}

Adolescents are considered to be those post puberty, but before maturity, generally aged 12-20 (Webster's, 2000). Adolescents make up a large sector of the population. This group remains one of the most underserved populations, especially the minority adolescent population, in terms of programs targeted at improving health (Freedman et al, 1998). Many of the health education-related resources available fall short of recommended practices especially in areas of preventive programs (Barlow et al, 2002). Adverse health risks, including elevated lipid levels, type 2 diabetes, and hypertension, are increasingly being noted among adolescents (Freedman et al, 1998). As a result, there is a need to increase awareness and to design educational programs/interventions that have the potential of improving the health of this group. Substantial evidence exists to demonstrate that adults can significantly reduce the burden of type 2 diabetes by changing dietary behavior and through the incorporation of daily physical activity (NIH, 2003). Interventions that begin before adulthood, therefore, have enormous potential to improve the nation's health status by influencing the behaviors young people will carry into adulthood (ADA, 2003). Furthermore, studies demonstrate that although adolescents' knowledge of healthy eating habits and consumption levels with regard to choosing healthy foods is low, their attitudes toward learning about healthier eating practices are favorable (Beech et al, 1999). 
The U.S. adolescent population has an increased risk of developing diabetes, heart disease, and other chronic diseases before becoming an adult (Diabetesincontrol.com. 2004). The financial burden associated with adolescent obesity and the precursors to chronic disease developed in adolescence have the potential to surpass our ability as a nation to treat these complications (Galvez et al, 2003). Moreover, on average, persons with diabetes incur medical expenses 2.4 times higher than those without diabetes (NIDDK, 2003). Estimates suggest that health care costs associated with chronic diseases, such as diabetes, related to overweight and obesity rose from $\$ 63$ billion in 1995 (NIDDK, 2001) to approximately \$132 billion in 2002 (NIDDK, 2003).

\section{Diet Quality}

Excesses and imbalances of nutrients have become the target for improving the health of adolescents in the US. The initiatives set forth by Healthy People 2010 specifically reflect these dietary imbalances and suggest a concerted effort be made to change these behaviors and "decrease the burden of preventable illnesses and premature deaths in the United States" (Healthy People 2010, 2000). The Healthier US initiative set forth by President George W. Bush in 2003 includes improving diet quality, increasing physical activity, and undergoing regular screening for chronic diseases such as cardiovascular disease (CVD) and diabetes (CDC, 2003).

The CDC reports that over $60 \%$ of young people eat greater than the recommended amount of fat and saturated fat, while only 34 percent of boys and 33 percent of girls consume the suggested 5 a day servings of fruits and vegetables each day. The Continuing Survey of Food Intakes by Individual (CFSII) in 1994-1996 estimated only $23 \%$ of girls and $27 \%$ of boys consume at least two servings of fruit per day, and 
only $7 \%$ of girls and $4 \%$ of boys consume at least 3 servings of vegetables (with at least 1 being dark green or orange) (Healthy People 2010, 2000). In addition, most do not consume the recommended amounts of fiber or grains (Jacobsen, 1999). The CFSII also estimated that although $77 \%$ of boys consume the recommended 6 or more servings of grains (with at least 2 being whole grains), only $49 \%$ of girls consume the recommended servings. The CSFII data indicates that adolescent males consume less than the recommended levels of vitamin E, magnesium, and zinc, while females do not meet the recommendations for vitamin $\mathrm{E}$, vitamin $\mathrm{A}$, phosphorus, magnesium, and zinc (CDC, 2002). Nearly $25 \%$ of the calories adolescents consume are from high fat, low nutritional value foods such as french fries, deep-fried foods, candy, and cookies (Jacobsen, 1999).

Frequency of family meals is positively associated with higher intakes of fruits, vegetables, grains and calcium-rich foods and negatively associated with soft drink consumption (Neumark et al, 2003), while adolescents eating away from home tend to consume a diet higher in fat, especially saturated fat, when eating away from home (Zoumas-Morse, 2001). Although improvements are steadily being made, the nutritional content of school lunch, which provides meals for an estimated $75 \%$ of public schools in the US, was found to have a mean fat percent of $41.3 \%$ and a mean percent of saturated fat of $16.1 \%$ (Jones et al, 1999).

Evidence suggests that intakes of calcium and iron are marginally low in adolescents, which is often the result of high consumption of convenience foods, fast foods, and sugary snacks (Spear, 2002). Low nutrient density snacks often crowd out more nutritious foods. From 1982 to 1990, the purchase of potato chips and similar snacks increased approximately 60 percent, with 75 percent of adolescents saying they 
eat at least 3 servings of chips per week. Nearly all surveyed said they would look for healthier alternatives if the price of chips were increased (Foodrun, 1999). A study by Serrano et al. (2005) revealed that when typical vending machine foods are replaced with healthier alternatives, there was a drop in purchases initially, however, after one month, sales were equal to baseline.

Consumption of calcium rich foods has declined, especially in girls (Jacobsen, 1999). Only $19 \%$ of girls meet the requirement for calcium, while $52 \%$ of boys meet the requirement (Healthy People 2010, 2000). The amount of milk purchased by school districts fell by nearly $30 \%$ from 1985 to 1997 . During the same time period, school districts purchased 1100\% more soft drinks (Moag-Stahlberg, 2004). Estimates suggest that nearly $18-20 \%$ of adolescents' caloric intake is derived from soft drinks, an average of 24 ounces (Murray et al, 2005), up from 15\% in 1999 (Jacobsen, 1999; Troiano et al, 2000). Approximately one-fourth of adolescents who consume soft drinks drink more than 30 ounces per day (Jacobsen, 1999). Pricing also encourages consumption of large quantities of soft drinks. A consumer can often more than double the size of the soft drink for less than half of the price (Jacobsen, 1999). More than $95 \%$ of the soft drinks have caffeine, which increases urinary calcium excretion, thus adding to the problem and impacting the growing adolescents' ability to achieve peak bone mass (Spear, 2002). The National Health and Examination Survey (NHANES) III indicated that adolescents aged 12-19 consumed, on average, between 796 to $822 \mathrm{mg}$ of calcium per day compared to the DRI for calcium of $1300 \mathrm{mg}$ per day (Johnson et al, 1994). With an already marginal to low intake coupled with the possibility of increased calcium excretion, bone health 
among this group may be in jeopardy. Overweight and obese adolescents have even higher soft drink consumption (Giammattei et al, 2003; St.Onge et al, 2003).

Meanwhile, decreased consumption of milk has had other types of impact on nutrient intake. Milk-drinkers not only consume more calcium but also more vitamin $\mathrm{B} 12$, riboflavin, folate, vitamins $\mathrm{A}, \mathrm{D}, \mathrm{B}_{6}$, and $\mathrm{B}_{12}$, and potassium (USDA, 2001). Even short-term deficiencies of these nutrients can have an effect on future risk for diseases (Lytle, 2002). Caffeine consumption has also been shown to have a negative effect on insulin sensitivity (Lee et al, 2005).

It is estimated that $2-4 \%$ of adolescent girls show evidence of true iron deficiency anemia while a high percentage are at risk of acquiring iron deficiency anemia (CDC, 2003). This is due to marginal intake and increased menstrual losses, as well as increased growth demand. Anemia may result in impairment of the immune response. Intake tends to be lower in adolescent girls than the general population. Whereas the recommended intake of iron is $15 \mathrm{mg} /$ day for girls, NHANES III indicates that most girls consume less than $12 \mathrm{mg}$ (Lytle, 2002). Fast foods from restaurants, convenience stores, and vending machines tend to be low in iron as well as calcium, riboflavin, vitamin $\mathrm{A}$, vitamin $\mathrm{C}$, and folic acid (Spear, 2002). Not only are these nutrients required for proper growth and development, diets low in these nutrients often include high calorie, low nutrient dense foods which are correlated with increased overweight and obesity (Nicklas, 2002). Overweight, Obesity, and Other Risk Factors for Chronic Disease

Overweight encompasses those individuals with a BMI in the range of 25-29.9, while obesity is reflected by individuals with a BMI $>30$. Obesity is currently considered the most prevalent nutritional disease of children and adolescents in the United States 
(Dietz, 1997). The prevalence of overweight and obesity for children 2-19 years old has increased by $182 \%$ from 1971 to 2000 and the extent of overweight has increased $247 \%$ over the same time period (Jolliffe, 2004). Overweight children and adolescents have an increased risk for cardiovascular diseases. Fatty streaks, elevated total cholesterol, reduced HDL, calcifications in the aorta and coronary arteries, and elevated blood pressure, all risk factors for cardiovascular disease, have been noted recently among children and adolescents (Freedman et al, 1999). As many as $2 / 3$ of obese children have one or more unfavorable cardiovascular risk factor (DiabetesinControl.com, 2004). Kimm et al reported a reduction of 1-2 points in the BMI lead to a significant improvement in risk factors associated with heart disease (dyslipidemia, hypertension, and insulin resistance) after participation in a nutrition intervention program over one year. In the same study, an increase in weight was associated with increasing disturbances in glucose metabolism (Kimm et al, 2005).

Overweight and obese children are at much greater risk for the development of type 2 diabetes (Fagot-Campagna, 2002). There is also a correlation between the progression of diabetes and the development of cardiovascular disease. Uncontrolled levels of serum glucose lead to the glycosylation of blood proteins that, over time, cause damage of vascular tissue (ADA, 2003). As a result, there is approximately a three-fold increase in the development of heart disease among those with diabetes compared to the non-diabetic population (Jones and Dewan, 2003). In adults, $80 \%$ of mortality of patients diagnosed with type 2 diabetes results from complications of heart disease (Jones and Dewan, 2003). In persons with diabetes, congestive heart failure (CHF) occurs 2.5 more often than in non-diabetics. These increases in risk demonstrate the importance of 
controlling modifiable risk factors for CHF such as obesity and hyperglycemia in one's younger years. Compared to those without diabetes, adults with newly diagnosed diabetes were 2.9 times more likely to experience a heart attack or related event (Jones and Dewan, 2003). .

The key endocrine organ that regulates insulin sensitivity in other tissues is adipose (Koistinen et al, 2004). Visceral fat is associated with whole body impaired glucose metabolism, including insulin resistance, and predisposes one to type 2 diabetes (Koistinen et al, 2004). It is has been suggested that the excess adipose tissue also plays a role in causing defects in insulin regulation of gene expression and likely contributes to the pathogenesis of type 2 diabetes. In children there is a positive association between visceral fat and abnormal lipid profiles. In other words, obesity not only leads to the development of type 2 diabetes but also manifests the complications that play a role in the morbidity and mortality of the disease (Koistinen et al, 2004; Gower, 1999).

Among overweight and obese adolescents, the percentage of caloric intake derived from fat is higher than for their normal weight counterparts (Garaulet et al, 2000). In a study to compare the effects of obesity, overweight, smoking, and problem drinking, it was shown that obesity has roughly the same association with chronic health conditions as does twenty years of aging; this greatly exceeds the associations of smoking or problem drinking (Sturm, 2002). It has been suggested that a child who is diagnosed with type 2 diabetes may be plagued with grave complications from the disease earlier in life along with a shortened life expectancy (Murray, 2005; Olshansky et al, 2005). The result can be a dramatic increase of health care costs. 
In adults, obesity is associated with a 36 percent increase in inpatient and outpatient spending and a 77 percent increase in medications. This compares with a 21 percent increase in inpatient and outpatient spending and a 28 percent increase in medications for current smokers; the coast associated with problem drinking are even less (Sturm, 2002). Furthermore, a 197\% increase in obesity-associated hospitalizations among adolescents in the past 20 years reflects the impact of the increasing severity of obesity (Wang and Dietz, 2003). Hospitalizations associated with obesity often lead to other medical conditions. As a result, the threefold increase totaling over $\$ 127$ million in hospital costs in 2001 has been reported (Wang and Dietz, 2002).

\section{Type 2 Diabetes}

In the past, type 2 diabetes was most often considered to be a disease of adults. Within the last decade the frequency of type 2 diabetes in adolescents and children has drastically increased (Steinberger and Daniels, 2003), with the incidence of new cases currently ranging from $8 \%$ to $45 \%$ depending upon the geographic location (Kaufman, 2002). In Florida, the new incidence rate among the adolescent population is estimated at approximately $20 \%$ (Macalusa, 2002). There are many theories surrounding this rapid increase. The increase has occurred too quickly to be caused by a change in gene frequency. Thus, more of an emphasis must be placed on the importance of environmental factors (Clement and Ferre, 2003; ADA, 2003). The increasing trend has paralleled the increase in prevalence and severity of overweight and obesity in children and adolescents (Fagot-Campagna, 2003). In addition, obesity has been implicated as a major contributor in the development of insulin resistance. Metabolic syndrome, which 
includes dyslipidemia, hypertension, and insulin resistance, has also drastically increased among obese children and adolescents.

A 2004 study revealed that $50 \%$ of severely obese and $39 \%$ of moderately obese adolescents had metabolic syndrome compared to an estimated $29 \%$ detected by NHANES from 1988-1994 (NJM, 2004). Up to 80\% of children and adolescents diagnosed with type 2 diabetes are overweight (Fagot-Campagna, 2003). Insulin resistance results in hyperinsulinemia. Long-term exposure to hyperinsulinemia may lead to loss of control of blood glucose and progressive development of type 2 diabetes. Studies indicate that overweight and obese adolescents have an abnormal "atherogenic" lipid profile and that the lipid profile is correlated with insulin resistance (Gungor et al, 2005). This "atherogenic profile," however, can be reduced with even moderate weight loss (Reinehr and Andler, 2004).

The Bogalusa Heart study demonstrated that those who were overweight or obese in adolescence were 2.4 to 7.1 times more likely to develop type 2 diabetes by age 30 . Seventy-seven percent of the Bogalusa Heart study participants who had a BMI over the $95^{\text {th }}$ percentile in adolescence were obese as adults (Freedman et al, 2001). [Figure 1] Nutrition intervention, including diabetes education and a variety of lifestyle improvement methods, has the potential to improve the health of the target group (ADA, 2003; Fagot-Campagna, 2001; NIH, 2003). Approximately 95\% of adolescents that have been diagnosed with Type 2 diabetes have a BMI greater than the $85^{\text {th }}$ percentile (Lytle, 2002). The age group 12-19 (during or past puberty) is described as the age group at highest risk for the development of type 2 diabetes (Fagot-Campagna et al, 2001). 
Figure 1. A Model to Illustrate the Progression of Chronic Disease in Adolescents

\section{Physical Inactivity \\ Poor Dietary Choices}
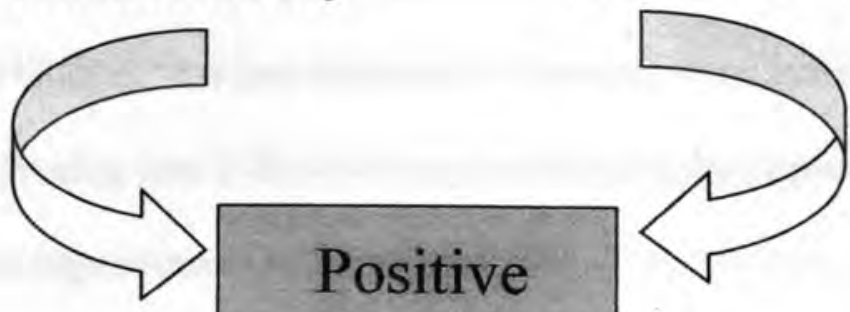

Positive

Energy

Balance

$\uparrow$ Risk of

Type

2 Diabetes

$\uparrow$ Body Mass Index

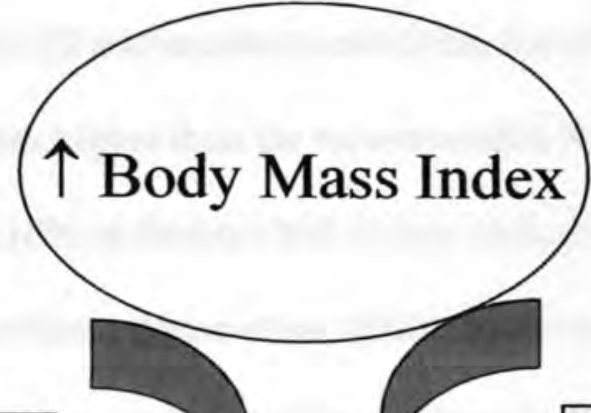

$\uparrow$ Risk of

CVD

Current estimates suggest that type 2 diabetes may be present for $9-12$ years

before diagnosis. By the time of diagnosis, many patients already have signs of

retinopathy and nephropathy, as well as two- to four-fold higher rates of development of

cardiovascular disease $(\mathrm{NIH}, 2003)$. Moreover, in adults, diabetes accounts for over $\$ 100$

billion annually in medical costs, with over $37 \%$ spent treating long-term complications 
(NIH, 2003). Diabetes is currently the leading cause of blindness, amputations, and kidney failure and drastically increases the risk of stroke (Conquering Diabetes, 1999). With the earlier onset of obesity and the complications of diabetes also beginning earlier, this number is likely to increase drastically. However, even individuals with the genetic propensity to develop type 2 diabetes may be able to delay or prevent the onset of the disease with an improvement in lifestyle habits.

\section{$\underline{\text { Gender Differences }}$}

In general, adolescents tend to make poor food choices, with significant differences noted between the genders. Compared to the American Heart Association guidelines, a study on 122 adolescents showed that the fat intake of $80 \%$ of the males and $73 \%$ of the females was higher than the recommended $30 \%$ of kilocalories. Thirty-seven percent of males and $16 \%$ of females had dietary cholesterol intakes above 300 milligrams (American Heart Association, 2000). More than 50\% of males and approximately $25 \%$ of females had sodium intakes greater than 3,000 milligrams. Mean dietary fiber intake of females was significantly lower than the fiber intake of males, but both were lower than recommended (CDC, 2000). In addition, females tended to skip meals (especially breakfast) more often than males (Story et al, 2002).

A study of 878 adolescents aged 11-15 revealed that girls had a higher risk for overweight and obesity if they were from a Hispanic or other minority background, independent of socioeconomic status (Patrick et al, 2004). Studies also demonstrate that females are more likely than males to be diagnosed with type 2 diabetes, and this increased risk occurs at a lower BMI than with males. Based on the analysis of 92 adolescents newly diagnosed with type 2 diabetes, females were at higher risk of 
developing type 2 diabetes when their BMI was greater than the $85^{\text {th }}$ percentile, while the risk for males did not greatly increase until their BMI was above the $95^{\text {th }}$ percentile (Macaluso et al, 2002).

Females have been shown throughout life to be inherently more insulin resistant than males (Wilkin et al, 2004). In a study to determine gender differences in insulin resistance $(n=1130)$, it was found that regardless of pubertal status, obese girls were more likely than obese boys to be insulin resistant and were at increased risk for the development of type 2 diabetes at an earlier age (Hirshler et al, 2003). Another study of 54 adolescents also indicated that girls had more adverse lipid profiles than boys. The girls had higher mean concentrations of total cholesterol and LDL than boys (Anding et al, 1996).

A recent study indicates that one of the primary reasons US adolescent girls are putting on weight is because they are doing less physical activity. Inactivity among girls was shown to result in three times greater gains in BMI and in weight gain of approximately $10-15$ pounds $(\mathrm{NIH}, 2005)$. Another study indicated that increased television viewing tends to have a greater effect on weight gain in girls than in boys (Koistinen et al, 2005).

\section{$\underline{\text { Racial Differences }}$}

The prevalence of overweight among non-Hispanic blacks and Hispanics has increased more than $10 \%$ over the past decade (Ogden et al, 2002). According to a national study, in the twelve years from 1986 to 1998 , the prevalence of overweight rose $50 \%$ for Caucasians children while increasing an alarming $120 \%$ among Hispanic and African American children (Strauss \& Pollack, 2001). African American and Hispanics 
are at higher risk for the development of overweight and obesity, with the prevalence increasing from approximately $19 \%$ at age 5 to $33 \%$ by age 17 --rates much higher than their Caucasian counterparts (USDHHS, 2000; Vander Wal and Thomas, 2004). For African American girls, higher weight is often considered a positive characteristic (Vander Wal and Thomas, 2004) despite initiatives that suggest negative health consequences. At least one author suggested that African Americans were less receptive to interventions that promote overweight prevention and were less likely to endorse eating and activity habits associated with weight loss (Dutton et al, 2004).

A decrease in physical activity throughout adolescence is more pronounced in black females than other females. In a recent study $32 \%$ of white girls kept up their childhood level of physical activity, compared to only $11 \%$ of black girls $(\mathrm{NIH}, 2005)$.

Advertising on television differs significantly between the African American and Caucasian viewing audience. Shows that are primarily geared toward the African American audience aired more advertisements for fast food, candy, soda, or meat and aired fewer ads for cereals, grains, pasta, fruits and vegetables. Marketing that promotes food of poor nutritional quality, particularly toward this audience, has the potential to further endanger a population already at higher risk for overweight, obesity, and the development of chronic diseases (Henderson and Kelly, 2005).

\section{Skipping meals}

Many adolescents do not consume the standard 3 meals a day, with breakfast reportedly the meal most often skipped (Nicklas et al, 2002). Although most adolescents believed that breakfast was the "most important meal of the day," $46 \%$ of the respondents in a study reported spending 5 minutes or less preparing a weekday breakfast (Nicklas et 
al, 2002). For example, the $1989-91$ CFSII data indicates that $20-24 \%$ of adolescents skipped breakfast entirely on the day of the survey. Another survey indicated that $40 \%$ of students in grades 8 to 10 ate breakfast 2 or fewer days each week (Story et al, 2002).

Studies show adolescents who eat breakfast score higher on tests, have better school attendance, have better diets, and may be less likely to be overweight (Butcher Powell et al, 2003; Ortgega et al, 1998). Moreover, adolescents that do not consume breakfast are more likely to be obese, since skipping breakfast often contributes to poor food choices for the rest of the day (Butcher-Powell et al, 2003). Skipping breakfast before school tends to increase consumption of vending machine foods, such as bakery goods and snacks, which can contribute as much as $58 \%$ of adolescents' total dietary fat intake (Wesnes et al, 2003; Nicklas, 1998). Finally, in adults, breakfast consumption has been correlated with the consumption of a lower percentage of cholesterol and fat on a daily basis, fewer inadequacies in their diet, and fewer tendencies to overeat (ButcherPowell et al, 2003).

\section{$\underline{\text { Socioeconomic Status }}$}

Most study samples investigating adolescent food habits come from teens that live with at least one parent, most often the mother. Consequently, it is often the mother who is responsible for food shopping and preparation. Nutrition knowledge of the mother is significantly and positively correlated with SES, her education and occupation level, housing conditions (quality and sanitation) and age, and significantly and inversely correlated with the number of children. Mother's education level and geographic area explains $93.2 \%$ of the variance in socioeconomic status and nutrition knowledge (Ivanovic, et al, 1997). 
The effect of socioeconomic status (SES) on lifestyle behaviors among children and adolescents is not well studied, as most of the data is extrapolated from adult studies. However, measures of SES, including education, income, and occupation are strongly associated with mortality and morbidity from CVD, cancer, and other chronic diseases; generally persons with a lower SES are at a greater risk for disease (Lowry et al, 1996).

The Youth Risk Behavior Survey (YRBS) conducted by the CDC is periodically administered to a random sample of $9^{\text {th }}$ to $12^{\text {th }}$ grade students in the United States and is used to monitor risk behaviors that contribute to the leading causes of death, disability and social problems among this population (CDC, 2000; YRBS, 2002). Data indicate that the risk of developing a chronic disease later in life is greatest among youth with parents who have less than 4 years of education and whose household income is less than $\$ 20,000$ (Lowry et al, 2000). Adolescents represent one of America's most underserved populations in terms of access to health services, with access being even less for those of lower SES. A high percentage of South Florida's student population (39\%) comes from low-income families (www.broward.org, 2004; School Board of Broward County, 2002). The effect of SES on health outcomes may result from a variety of societal and individual factors including housing, nutrition, social support networks, access to public health care, and adoption of lifestyle behaviors that are associated with illness and premature death (Lowry et al, 2000).

Under-funded schools receive extra income from vending machines, providing an incentive to market soda and snacks to students (Nestle, 2000). Ludwig et al (2001) indicated that the risk of obesity increases $60 \%$ for each extra $8 \mathrm{oz}$. serving of sugared soft drink consumed per day. 
While malnutrition in developing countries is unquestionably linked to environmental factors, the scrutiny of the environmental role in over-nutrition is now being recognized. The "concrete environment" (densely populated, economically skewed, psychosocial stressors, and lack of play spaces) can have drastic effects on the growth and development of children and adolescents. This is further evidenced in lowincome predominantly minority communities (Jackson, 2003). Further study on the impact the "concrete environment" has on lifestyle behaviors is warranted. Although the individual is ultimately responsible for making choices, environmental exposure often promotes poor dietary habits and physically inactive lifestyles.

As adolescents age, they become more independent and as a result rely less on "home-cooked meals." The taste appeal of fast foods, coupled with the ease with which it can be obtained, clearly has an impact on consumption. If intervention programs are to be effective they must address ways in which healthier alternatives can be procured, prepared, and stored while being cost effective. In addition, teaching adolescents how to select wisely at fast food restaurants is needed.

\section{Physical Activity}

The leading health indicators as put forth by the Healthy People 2010 (HP 2010) suggest that individual behaviors, environmental factors, and health system issues affect the health of individuals and communities. Two of the leading indicators of HP 2010 are physical activity and overweight/obesity. Consequently one of the main objectives of the initiative is to "increase the proportion of adolescents who engage in vigorous physical activity that promotes cardio-respiratory fitness 3 or more days a week for 20 minutes or more per occasion" (Healthy People 2010, 2000). It is clear that, despite their natural 
tendencies, children have become less physically active in recent decades, with children today expending approximately $600 \mathrm{kcal}$ per day less than their counterparts 50 years ago (Boreham and Riddoch, 2001). It is estimated that children and adolescents who spend 2 or more hours a day doing sedentary activity have an increased risk of high cholesterol, diabetes, poor fitness, and being overweight in adulthood (Hancox et al, 2004). Being overweight further exacerbates the aforementioned risk factors and decreases the likelihood that the person will engage in physical activity (Blanchard et al, 2005).

Making physical activity part of a daily or weekly routine is a strong predictor of long-term weight management. Physical activity, although not solely responsible for weight management, does assist in weight loss, as it improves body composition, body image, psychological well-being, and feeling of control over eating behavior (Foreyt, 1999). In addition, regular physical activity in teens and adolescents is proven to be beneficial to improved strength and aerobic endurance, healthier bones and increased bone mass and density, weight and maintenance of healthy joints (United States Department of Health and Human Services, 2000). Physical activity has also been shown, even in the absence of dieting, to improve visceral fat distribution, BMI and insulin sensitivity and to decrease blood pressure and raise HDL cholesterol (Di Loreto et al, 2005; Giannopoulou, 2004).

Despite these benefits, nearly 50 percent of Americans aged 12 to 21 are not vigorously active on a regular basis. There is a sharp decline in activity during adolescence. Studies report that from $9^{\text {th }}$ grade to $12^{\text {th }}$ grade, there is an approximate 25 percent decrease in participation of regular physical activity (USDHHS, 1999). Patrick et al (2004) concluded that failing to meet the daily guideline of moderate to vigorous 
physical activity was associated with overweight status in both girls and boys. In general, fewer girls than boys are physically active, and the gap widens with physical maturity. Kettaneh et al, (2005) found that all adiposity indicators were higher in girls who had decreased their level of moderate physical activity. Most secondary school-based physical activities are centered on competition. Studies show that many girls prefer activities that allow them to work together to accomplish goals, rather than competitive activities such as large-group sports (Wiese-Bjornstal, 1997). Due to lack of resources within the schools and overpopulated classes, traditional large group activities are often used. Often, these large group activities do little to develop motor skills and do not afford the benefits that are needed to establish lifelong physical activity patterns (Arnett, 2001; Lambert, 2000). It is this "one size fits all" approach to physical education that may lead to lack of enjoyment of these activities and even an avoidance of participation (Arnett, 2002). Additionally, large class sizes and limited resources result in lack of actual participation.

Physical activity patterns also have an association with race. African Americans and Hispanics tend to be less active than their Caucasian peers. They are also the groups that are at the highest risk for obesity (Lytle et al, 2000; Freedman et al, 1997) and the development of type 2 diabetes (Fagot-Campagna, 2002).

In general, people gain weight when there is an imbalance of calories due to an increase in the amount of calories consumed or there is a decrease in the amount of calories expended (Cutler et al, 2003). Current research indicates that both are occurring simultaneously. As a result, the obesity epidemic has spread and is currently considered the number one nutritional disease of childhood and adolescence (Dietz, 1997). Weight 
gain in adolescence poses a few more problems since some weight gain is necessary as the adolescent grows and matures. However, excessive weight gain and weight gain that surmounts normal growth weight gain is a serious issue in this age group (Jones and Dewan, 2003).

In adults, participation in physical activity has been linked with reduced risk of all causes mortality and decreases in heart disease, incidence of type 2 diabetes, colon cancer, hypertension, hyperlipidemia, and insulin resistance (Jensen, 1998). Physical activity may also decrease depression, and anxiety and increase overall mental health (Jensen, 1998).

Yet, despite these proven benefits, approximately two-thirds of American adults do not exercise regularly while one-quarter is sedentary. Similar trends are seen in adolescents. During what was once time for physical activity, adolescents now do sedentary "screen time" activities such as viewing television, using the computer, or playing video games. Consequently, it has been hypothesized that television viewing and other sedentary activities cause obesity by one or a combination of three mechanisms: (1) displacement of physical activity; (2) increased calorie consumption while watching television as a result of the effects of advertising; and (3) reduced resting metabolism (Robinson, 2001). If this rationale that television viewing and physical inactivity are causes of increased body fatness is correct, then reducing sedentary activities while increasing participation in regular physical activity is a promising strategy for preventing childhood obesity (Jago et al, 2005; Robinson, 2001).

Recent evidence suggests that more active children generally display healthier cardiovascular profiles, are leaner and develop higher peak bone mass than their less 
active counterparts. Also, there is a biological and a behavioral carry-over effect into adulthood, whereby improved adult health status results from childhood physical activity and increased activity in adulthood (Boreham and Riddoch, 2001).

\section{Intervention Programs}

\section{Nutrition Education}

Schools are not the primary source of the obesity problem, yet schools can contribute to curbing the obesity problem. Increasingly, children and adolescents consume at least one, and often two, of their meals at school (Dalton, 2004). Offering students tips and teaching them strategies that may be implemented in their daily lives in and outside of school has the potential to improve life-long health (Holcomb et al, 1998). The classroom offers an excellent opportunity for students to learn about the importance of good nutrition and physical activity in promoting health. School can also promote healthy food choices and opportunities for physical activity. Health professionals have to reclaim the responsibility of educating young children. It is the position of the American Dietetic Association (ADA), the Society for Nutrition Education (SNE), and the American School Foods Service Association (ASFSA) that all of the nation's schoolchildren should have access to comprehensive, coordinated school nutrition services that support positive health outcomes (Briggs et al, 2003). Providing nutrition education at an early age reaches students when they are beginning to make independent decisions about dietary behaviors. Obesity prevention is especially important during early adolescence years because juvenile obesity may begin or worsen during this period of accelerated growth. Although overweight adolescents tend to acquire a greater percentage of their caloric intake from fat and less from complex carbohydrates than 
normal weight adolescents (Garaulet et al, 2000), a study of 292 high school adolescents illustrated that overall nutrition knowledge did not differ between obese and non-obese adolescents (Thakur and D'Amico, 1999). Additionally, other macronutrients such as protein and simple carbohydrate intake are similar among obese and non-obese adolescents, as is micronutrient intake (Gordon-Larson, 2001).

Studies demonstrate that although adolescents' knowledge of healthy eating habits and consumption of fruits and vegetables are low, their attitudes toward learning about healthier eating practices are favorable (Beech et al, 1999; Reynolds et al, 1999). Interventions that include nutrition, physical activity, and overall health education therefore have the potential to improve lifestyle habits and influence the future health of adolescents (Fagot-Campagna, 2001; ADA, 2003; NIH, 2003). Even individuals that have the genetic propensity to develop diseases such as type 2 diabetes or cardiovascular disease may be able to delay or prevent the onset of disease with improved lifestyle habits. Nevertheless, providing effective preventive services to this population presents many challenges (Skinner et al, 2003).

Traditional nutrition education models emphasize increasing the knowledge of participants, with the rationale that increased knowledge will lead to behavior changes (Hoelsher et al, 2002). However, such programs have done little to elicit change in food/nutrient intake (Hoelsher et al, 2002; Sallis et al, 2003; Reynolds et al, 1998). In reviewing several general nutrition intervention programs, researchers have concluded that a behavioral focus, as well as incorporation of appropriate instruction, is necessary for intervention effectiveness (Hoelsher et al, 2002; Lytle, 2002). Nevertheless, interventions tend to use a "one size fits all" traditional approach of didactic teaching by 
telling. The most effective solution lies in a comprehensive approach that enables, encourages, and fosters individual change in behavior.

\section{Barriers to Nutrition Education}

When surveyed, school administrators indicated that the main barriers precluding preventive programs in the schools were lack of trained personnel, materials, classroom time, funds, and staff time (Dalton, 2004). These barriers are even more pronounced in school districts comprised of students with low socioeconomic status. Students typically name time, money, and availability as major barriers to attaining a healthy lifestyle (Bauer et al, 2004; McKinnley et al 2005). Additionally, few adolescents recognize dietary intake as it relates to health status (Glanz, 1997). Only one in three adolescents report that nutrition is very important in relationship to health (Waterman, 1998). Therefore, education efforts need to incorporate the relationship of today's lifestyle behaviors with present and future health. Consequently, better selections including increased fruit and vegetable intake, decreased fatty foods, and decreased junk and fast foods need to be addressed. Many fast food restaurants offer nutrition facts and therefore provide information about healthier alternatives. However, some of these "supposed" healthier alternatives can be deceiving if one takes into account added fats such as toppings and mayonnaise. Consequently, it is essential that adolescent consumers be aware of the nutrient content of foods they eat.

Adolescents, in general, do not read food nutrition labels and are generally unable to estimate caloric or fat content (McCullum and Achterberg, 1997; Shannon et al, 2002). Nevertheless, in adults, there is a highly significant association between reading nutrition labels and eating a healthier/lower-fat diet, especially among women (Neuhouser et al, 
1999). This evidence suggests that a person who wishes to reduce fat intake should use nutrition labels to help them select lower fat, higher fiber foods. There is a little scientific evidence regarding adolescent label reading and its effect on food selection but it is commonly assumed that adolescents pay little attention to information on food labels or have a lack of experience in interpreting these labels (McCullum and Achterberg, 1997; Shannon et al, 2002).

A number of nutrition interventions based on learning theories have been developed to help individuals change diet and physical activity behaviors, including strategies for identifying and overcoming barriers that prevent a healthy diet and increased physical activity (VanWormer and Boucher, 2004; Lytle, 1995; Hoelsher, 2002). No single strategy or combination of strategies has been shown to be superior over another (Foreyt, 1999).

The most modern approach, which began in the 1980s, consists of school based intervention programs that focus on reducing risk factors for chronic disease (Lytle, 1995). Research indicates that nutrition education is more likely to be effective when it is behaviorally focused, i.e. consumption of targeted foods, or decreased fat intake compared to general nutrition education (Hoelsher, 2002; Lytle, 1995) and relevant to behaviors for the target population (Lytle, 1995). In order for the curriculum to be effective, the information must have relevance to the individual student and able to be integrated and transferred to the students' own lifestyles (Oliva, 2001). This is often difficult to accomplish using standard printed material via traditional education. However, computer based programs provide a powerful medium because programs offer fast, low-cost access to information without geographic barriers (Kolasa and Miller, 
1996). In addition, learners are able to access and assimilate information at their own pace.

\section{Computer-Based Learning}

Changes are occurring in many areas relevant to nutrition, including health care, education, and technology. The United States Department of Education reports that personal computer use has increased every year since 1994 (Wright, 2001). This creates important opportunities for engaging young people in computer-based health programs (Skinner, 2003). A 1998 survey reported that 71 percent of U.S. households with children 8-17 had computers; this figure continues to grow annually (Wright, 2001). Computer-mediated communications such as e-mail, websites, and CD-ROM and DVD programs, are beginning to assume a large role in the future of behavioral health care, a fact that has huge implications for health educators (Probst \& Tapsell, 2005; Brug et al, 2003; Oenema et al, 2005).

Traditional teaching involves teaching didactic instructions. In this "information age," however, the traditional teaching approach does not appear to parallel the learning style preferred by most of today's students (Litchfield et al, 2002). Today's adolescents, as part of the "net generation," expect learning to be not only fun and entertaining, but also customized, flexible, and immediate (Wright, 2001). It is estimated that between 80$90 \%$ of what we learn is visual (Jensen, 2000). Our brain gives priority to color, light, movement, form, and depth and has an immediate and basic response to symbols, icons, and other simple images (Jensen, 2000). The combination of these factors often cannot be attained using traditional methods of teaching. A computer-based dissemination of information, however, has the potential to more accurately reflect the brain's ability to 
process information. Therefore, computer-based instruction has the ability to improve the learning environment by offering a variety of information media with an array of colors and pictures that also incorporate motion.

Several longitudinal studies have demonstrated the benefits of instructional technology such as the Dole 5 A Day ${ }^{\circledR}$, StampSmart $^{\circledR}$, Project Pegasus ${ }^{\circledR}$, and Apple ${ }^{\circledR}$ in other age groups and other disciplines (Wright, 2001). The Apple Classrooms of Tomorrow (ACOT)® demonstrated that technology in the classroom has the ability to significantly increase the potential for learning (Wright, 2001). Results suggest that interactive, computer-delivered, programmed instruction can be very important to improving outcomes in the high school setting (Kreisel, 2004; Kumar et al, 1993). These computer-based interventions go beyond simply providing information for the sake of knowledge gain. They can include strategies to incorporate healthy behaviors into the daily lives of adolescents and focus on actions needed to modify behavior by providing an interactive approach as well as instant feedback. Participants utilizing computer-based instruction play an active role in their own learning process compared to passive learning in traditional education models (Kreisel, 2004; Kumar et al, 1993; Wright, 2001; Beerman, 1996). Moreover, hardware and software capabilities can be expanded to provide a means for charting physical activity, analyzing nutrient quality of meals, providing a feedback forum, as well as an opportunity for communication between peers.

Evidence illustrates that computer-based learning has worked in other populations and in other areas. Many children are learning to apply basic school skills (reading, writing, and arithmetic) to problem solving with the assistance of interactive computer programs. Beerman (1996) concluded that computer-based teaching helps students 
maintain interest in the class and helps students learn material more effectively. Using the Squire's Quest! ${ }^{\circledR}$ elementary school program $(n=1578)$ the intervention group that used a computer-based approach increased their fruit, juice, and vegetable consumption by 1.0 serving more than the control group. The authors concluded that this type of program has the potential to substantially change behavior (Baranowski et al, 2003). This research suggests that computer-based health promotion programs are likely to be effective educational interventions.

\section{Applications}

Adolescents remain one of the most underserved populations, especially minority youth. Many of the health education-related resources available do not adequately address the recommended preventive practices (Skinner et al, 2003). Although past interventions have resulted in increases in knowledge, the ultimate goal of health education is to positively influence lifestyle behavior and enhance health through the reduction of risk factors (Hoelsher et al, 2002; Litchfield et al, 2002).

A behavioral focus addresses the domains of learning the "how," the factors that motivate one to change current lifestyle patterns, and assists in building new skills and behaviors. Although there is evidence that several factors independently contribute to the development of chronic diseases such as type 2 diabetes or heart disease, the results of several prevention studies in adults indicate that there are now interventions that are successful in eliciting behavior changes that promote a reduced incidence of chronic diseases, or at least delay onset of these diseases (ADA, 2004). In order to be effective, however, the knowledge and skills must be learned so that the targeted behaviors can be incorporated into the lifestyle of the target group. Programs that facilitate not only the 
understanding of proper nutrition and the need for physical activity, but also elicit a change in dietary and physical activity behaviors among adolescents are necessary to enhance the health and well being of this group. Such interventions will offer us as educators a means to provide relevant information that allows for individualized strategies to promote weight management, physical activity, healthy eating habits, and may result in improved health status for this population.

However, the "one size fits all," "teaching by telling" approach does not seem to fit the learning style of today's adolescents. On the other hand, the use of technologically enhanced resources, such as multi-media websites, CD-ROMs, or other computer-based programs which incorporate a behavioral focus have been shown to have a greater impact in motivating people of all ages to change their diet, in particular their fat and consequently caloric intake, compared to more traditional intervention methods (Budman, 2000; Brug et al, 1999). Consequently, the American Dietetic Association and the American Diabetes Association have stated that effective, behaviorally focused programs emphasizing lifestyle modification are likely to have substantial health benefits (Skinner et al, 2003). Past interventions that have followed the traditional approach have been unsuccessful in eliciting a behavior change. There is a compelling need for innovative approaches to nutrition education. Perhaps the effectiveness that we are seeking is dependent upon a more behaviorally based way of delivering the information to this population. A method that parallels the learning styles along with the advances in technology seems an obvious choice for this delivery. 


\section{Using the Practical Approach}

Chronic disease prevention involves changing a complex set of behavior patterns. Group norms and the environment are especially important in youth whose cognitive processes have not fully developed. A program to target health risk must be behaviorally based, supported by practical data, and tailored to the needs and beliefs of the target community (Burnet et al, 2002). Lifestyle behaviors are influenced by social norms and constrained by social, economic, and environmental factors as well as systemic barriers that minimize access to healthcare and often create a barrier to behavior change in this population. The aforementioned barriers that exist are elevated in minority youth.

Several models have been developed in an attempt to elicit a behavior change and encourage adolescents to adopt healthier dietary and physical activity patterns. For example, the Knowledge, Attitudes, Behavior (KAB) model was found to be ineffective in changing behavior in adolescents despite increases in knowledge (Contento, 1995). The authors concluded that there was a need to develop behavioral skills in desired areas in addition to simply increasing knowledge. Moreover, the Health Belief Model (HBM) suggests that health behavior depends on perceived susceptibility to and perceived severity of a health problem, and the belief that changing a behavior will reduce susceptibility (Glanz, 1996). Youth, typically do not feel vulnerable to the risk of chronic disease therefore the HBM has limited value for this group (Burnet et al, 2002; Glanz, 1996). The Social Cognitive Theory hypothesizes that a behavior change will occur if there is a valued outcome. The underlying premise of the theory is that the targeted group is capable of making the change, and incentives exist to make the behavior change. Again, there has been limited success with this approach in the adolescent population 
(Burnet et al, 2002). Finally, the Theory of Planned Behavior suggests that a person will attempt to perform a behavior if they believe that the advantages outweigh the disadvantages and others will value the behavior. All of these theories have important and useful constructs, yet none have been shown to be vastly effective in adolescents (Burnet et al, 2002).

It has been suggested that in order for dietary interventions to be successful in the behavioral change process it must provide at least three components: the cognitive, the affective, and the behavioral (Contento, 1995; George, 1998). The cognitive component focuses on knowledge and comprehension of the behaviors to be incorporated. This includes predisposing the population to pertinent information and providing concurrent health messages. However, an increase in knowledge alone does not necessarily equal to a change in behavior and therefore requires additional provisions. The affective component is aimed at addressing beliefs, attitudes, and perceptions associated with healthy lifestyle behaviors. The practical approach aims to empower individuals in an effort to get them to take responsibility for the choices that they make now in an effort to alleviate chronic disease later in life. In doing so their perceptions, beliefs, and attitudes toward a healthier lifestyle as well as barriers that prevent the adoption of a healthy lifestyle must be addressed.

\section{Empowerment}

The nature of a health behavior approach that includes empowerment assists in maximizing healthy lifestyle development, identifies, clarifies, and interprets healthy and unhealthy lifestyle behaviors, and incorporates strategies in managing daily life.

Empowering adolescents involves teaching them how to take control of their lives and 
actively engage in behavior change that will have a life-long effect on health status.

Efforts need to be directed toward providing education and skill building based on beliefs and life situations (i.e., food selection and preparation) (Krummel et al, 2002).

Adolescents often do not feel that there is a link between present behavior and future health. In addition, there is a perception that health status is predetermined and cannot be modified. For example, Jain et al (2001) found that body size and risk factors for chronic disease associated with body size was thought to be predestined and therefore impossible to alter. Empowerment strategies foster strengths and promote positive health behavior development and help adolescents recognize personal strategies used to overcome perceived health powerlessness.

Self-Efficacy

Self-efficacy, first conceptualized by Bandura in 1977, is the belief in one's ability to perform a certain task (Britner and Pajares, 2001). Individuals that doubt their ability to make a behavior change are more likely to avoid tasks associated with these changes, set low goals, and make minimal commitment to these goals (Bandura, 1986). An adolescent's belief about his/her capability to produce effects, or self-efficacy, can have a tremendous impact on one's ability to make a behavior change relating to diet and physical activity. In general, people who have higher self-efficacy are more willing to try something new, more likely to persist until they succeed, and more likely to resist episodes of relapse (Bandura, 1986).

Multi-component chronic disease prevention programs attempt to establish changes to health behaviors while also striving to influence beliefs, self-expectancies, and skills required for sustained healthful behavior. However, adolescents who doubt their 
ability to make these changes, especially if they have attempted before, will dwell on personal deficiencies, adverse outcomes, and obstacles they will encounter. In such programs, setting realistic goals and reviewing progress can increase a person's selfefficacy through performance accomplishments.

Health promotion interventions intended to improve dietary behavior frequently incorporate self-efficacy as a construct to enhance a behavior change in adults but have remained relatively unexplored among children and adolescents (Parcel, et al, 1995). However, it is well documented that a strong sense of self-efficacy enhances behavior change (Bandura, 1994). With the lack of success of dietary and physical activity interventions in eliciting behavior changes in this group, a program that specifically addresses self-efficacy may be a crucial part of chronic disease prevention among adolescents (Parcel et al, 1995). The degree of control an adolescent has over their own food environment is a potentially modifiable predictor of their fat intake. Interventions may benefit from involving the child or adolescent in their own nutritional decision making process.

The Child and Adolescent Trial on Cardiovascular Health (CATCH) intervention included a successful self-efficacy component and indicated self-efficacy as a strong predictor of food choices among the sample (Parcel et al, 1995). Students who have a strong sense of self-efficacy are better equipped to educate themselves when they have to rely on their own initiative (Britner and Pajares, 2001). In order to strengthen selfefficacy, adolescents require adequate training in the skills needed to perform behaviors confidently on their own. If promotion efforts fail to build self-efficacy within individuals desiring change, the new behaviors may last only as long as the program 
exerts its external pressures. In a recent study involving two junior high schools, selfefficacy was significantly associated with dietary knowledge of fat content of food and choices of foods lower in fat (Long and Stevens, 2004).

\section{Social Support}

Social influences such a family and peers (including interpersonal and community factors) are especially important to adolescents whose knowledge, beliefs, and eating habits are strongly influenced by the environment. Relationships with others have a strong influence on behavior as these relationships provide models for change, controls and constraints to behavior, access to information, and a sense of meaning that can help make healthy lifestyle changes (diet and physical activity) more attainable (Blanchard et al, 2005). Having people who are supportive in one's life is critical to initiating and maintaining the process of behavior change. Support is most successfully shared among individuals who are socially similar, have common experiences, coped with similar stressors and situations, and have similar personal attributes. Developing social linkages by encouraging people to be accountable to others, develop partnerships and buddy system, establish a means to create a dialog between people and their social network, and build self-esteem and self-efficacy increases the likelihood of behavior change.

Moreover, research has shown that the social support in regards to diet offered by youth to others is significantly related to reduction in their own unhealthy dietary behaviors after a 3-month intervention (Nader, 1993).

Other studies have also validated the importance of social support in behavior change. Behavior change was positively reinforced by social support and validation in women 33-82 years old in a study that utilized Margaret Newman's Theory of Health as 
Expanding Consciousness (Berry, 2003). It was concluded that people who are persuaded verbally that they possess the capabilities to master a given activity are likely to put forth a greater effort to attain a goal and sustain it. Social support also enhances self-efficacy as well as promotes the development of skills (Bandura, 1994) helping them to structure situations that help bring success and avoids failure.

Peer influence tends to increase with age and thus increasing peer support may have a profound effect on the success of interventions aimed at improving the lifestyle patterns of this group. Using the social support scale developed by Sallis, researchers determined that peer support was the strongest correlate of self-reported physical activity after controlling for gender, grade, and race (Prochaska et al, 2002).

\section{$\underline{\text { Behavioral Focus }}$}

The goal of a behaviorally focused health education program is to enhance health through the reduction of risk factors (Hoelsher et al, 2002). Although past interventions have seen increases in knowledge, the ultimate goal of a health education program is to impact behavior. A behavioral focus addresses the domains of motivating one to change current lifestyle patterns, teaching the "how" of the new or preferred behavior, and assisting in building new skills and patterns (Journal of the American Dietetic Association, 1999). Although there is evidence that several factors independently contribute to the development of chronic diseases such as type 2 diabetes or heart disease, the results of several prevention studies in adults indicate that there are now interventions to support changes at least delaying the onset and reduce the incidences of these diseases (ADA, 2004). These lifestyle changes included moderate exercise of $30 \mathrm{~min} /$ day as well as improvements in dietary choices to accomplish weight reduction. Improvements in 
risk factors associated with type 2 diabetes as well as improvements in cardiovascular health were noted in subjects in this study (ADA, 2004). Consequently, the American Diabetes Association has concluded that behaviorally focused programs emphasizing lifestyle modification are likely to have substantial health benefits (ADA, 2004). Even modest changes of $5-10 \%$ in body weight and mild to moderate (20 to 60 minute) increases in daily physical activity have been shown to lower risk factors for chronic disease (ADA, 2004).

\section{Purpose}

The purpose of this research was to determine whether a behaviorally oriented computer based intervention (CBI) program using an interactive, animated CD-ROM would elicit a greater change in modifiable risk factors for chronic disease in high school students than would a traditional didactic intervention (TDI) program. Both education programs emphasize healthy lifestyle habits (diet and physical activity). A third group served as a control and did not receive any intervention.

\section{Research Questions}

1. Will high school students gain more nutrition knowledge if a behaviorally oriented nutrition education intervention is delivered using an interactive animated CD-ROM than if it delivered via a traditional didactic intervention program?

Hypothesis 1: The CBI group will show a greater increase in knowledge compared to both the TDI and control groups post-intervention and at follow-up.

2. Will high school students benefit from a greater behavior change if a behaviorally oriented nutrition education intervention is delivered using an interactive animated CDROM than if it delivered via a traditional didactic intervention program? 
Hypothesis 2: The CBI group will have greater success in maintaining/ attaining a healthy body mass index compared to both the TDI and control groups postintervention and at follow-up.

$>$ Hypothesis 3: The CBI group will show a decrease in fat intake, especially saturated fat, an increase in fruit, vegetable, and fiber intake, and increased consumption of low-fat dairy products compared to both the TDI and control groups post-intervention and at follow-up.

$>$ Hypothesis 4: The CBI group will have an increase in physical activity level compared to both the TDI and control groups post-intervention and at follow-up.

$>$ Hypothesis 5: The CBI group will increase their frequency of food label reading compared to both the TDI and control groups post-intervention and at follow-up.

3. Will high school students have greater changes in variables that can affect dietary behaviors if a behaviorally oriented nutrition education intervention is delivered using an interactive animated CD-ROM than if it delivered via a traditional didactic intervention program?

Hypothesis 6: The CBI group will increase their self-efficacy of dietary and physical activity habits compared to both the TDI and control groups postintervention and at follow-up.

$>$ Hypothesis 7: The CBI group will increase their perceived social support compared to both the TDI and control groups post-intervention and at follow-up. 


\section{Chapter III \\ Research Methodology}

\section{Development of Behaviorally Oriented Intervention Program}

Two qualitative studies and one quantitative preliminary study were completed to develop and test feasibility of the intervention. These studies attempted to ascertain what influences typical food choices high school students made on a daily basis, how they connected eating behaviors with overall health status, the actual type of foods they were eating, and where they obtained the foods that they typically ate. The qualitative studies were implemented several months before the actual intervention was developed and the information gathered helped shape the intervention developed for use in this study. The quantitative study was used as a pilot test for the intervention. It was tested on a group of high school students of similar demographic background and tested the usability and feasibility of the CD-ROM used in the CBI group as well as content used in the TDI group.

\section{Preliminary Qualitative Studies Used in Tailoring of Lifestyle Methods Before}

\section{Initiation of Research Study}

A qualitative study $(\mathrm{n}=16)$ was completed to determine, from the adolescent's perspective, why they chose the foods they did, the health consequences they associated with these choices, and the barriers they perceived as preventing improvement of their diet quality. The Institutional Review Board at FIU approved the study.

Participants were aged 13-18 (mean $n_{\mathrm{age}}=15.6$ years) and were enrolled in the Broward County Public School System. Nine females and 7 males were asked a series of 6 open-ended questions in order to obtain information on their typical daily dietary 
intake, the reasons they selected the foods that they ate, and how they felt their diet related to their health status. Transcripts of written interview responses, lists of factors perceived as influencing food choices, and observations of individuals were all incorporated into the analysis process. The data were analyzed to determine what lifestyle (dietary and physical activity) behaviors were taking place, what lifestyle choices were in need of modification, and perceived barriers to modifying these behaviors. Once this was determined, a relevant and practical approach could be developed to specifically target this population.

Several factors emerged as possible influences on food choices, the largest being on taste, followed by availability, time constraints, and money. These results were similar to those found by Neumark-Sztainer et al (1999). Only two adolescents indicated that the choices they made were due to the health implications associated with chosen foods.

All subjects except one (94\%), indicated knowing the relationship between diet and health, however, there was a lack of urgency about future chronic disease prevention and students did not appear to fully understand the actual relationship between diet and disease. Several (63\%) indicated that they could improve their diet [sic] by exercising. Others (44\%) indicated that eating more fruits and vegetables would improve their diet but could not explain why. The subjects also were not aware of any problems associated with skipping meals. For example, although $63 \%$ indicated that they did not consume breakfast on the day that they were surveyed, only $50 \%$ of these same students indicated that they felt their diet needed improvement. Programs need to have both health and dietary components that are relevant to adolescents such as improved eating behaviors 
and success in school activities or athletics. Although, the adolescents in this study wanted food to be prepared for them, knowledge of easy and quick ways to prepare meals for themselves may be of tremendous benefit now, and in the future.

Information from the qualitative study was used to tailor the nutrition education messages for the intervention program to the target audience in the main study. Understanding why adolescents make the food choices they do can lead to eliminating the barriers that prevent more healthful dietary behaviors.

A second qualitative study $(n=8)$ was conducted to determine what types of meals teens eat and where they obtain their food. The Institutional Review Board at FIU approved the study. In order to develop effective intervention programs to change the dietary habits of this group, it is important to be aware not only of the types of foods adolescents eat, but also where they obtain the foods they consume.

This study revealed that much of the food eaten by these adolescents was purchased in school and eaten during school hours. Nearly $40 \%$ skipped breakfast on the day of the survey. Four (50\%) of the students obtained their lunch from the school cafeteria indicating that they usually choose from the "pizza line" or "sub sandwich line." Additionally, six (75\%) utilized a vending machine each day either to get "lunch" or a snack. Items that were chosen included candy, pastries, chips, soda, fruit punch, cookies, popcorn, and granola bars. Half $(n=4)$ of the students reported eating a dinner meal prepared by a parent, while three reported ordering fast food most nights, and one student reported that he did not usually consume dinner "because there is usually nothing to eat." Most reported that they usually had a dessert following dinner such as ice cream, cookies, or "icees." One student indicated that she had drastically changed her eating habits as she 
had been diagnosed with type 2 diabetes during the past month. She stated, "I can't eat anything that I like anymore; no more Cheetos ${ }^{\circledR}$, Snickers ${ }^{\circledR}$, or McDonald's®.”

\section{Intervention Approach- Practical Lifestyle Approach to New Behaviors (PLAN B)}

Studies demonstrate that an increase in knowledge alone is effective in predisposing the target audience to the information but is not effective in eliciting the desired behavior change (Lytle, 2000). Behavior modification involves the interaction of several factors, all of which can play a major role in the behavior change process. There are a variety of models that have been applied to the behavior change process. The practical approach used in this intervention is based on constructs from a variety of these models such as: the Proactive Model (George, 1998), the Solution Model (Mellin, 1997) the Transtheoretical Model of Change (Prochaska, 1979), and theories including: the Empowerment Theory (Glanz et al., 1996), the Social Cognitive Theory (Bandura, 1986), and the Theory of Reasoned Action/ Theory of Planned Behavior (Fishbein, 1993).

Figure 2 illustrates the interaction between factors and impacting variables that can affect behavior modification, and how this approach addresses each in attempting to change the lifestyle habits of the participants. The major constructs taken from the aforementioned theories and models have components which include increasing awareness, building a social support network, taking responsibility for one's own health, and offering tips and strategies that are relevant to the lives of this group of adolescents.

The educational component addressed nutrition knowledge, the importance of physical activity, and how diet and physical activity can have an effect on the development of chronic diseases later in life. Participants were taught how to apply this information to label reading, planning menus that still include foods they enjoy, yet are 
Figure 2. The practical approach.

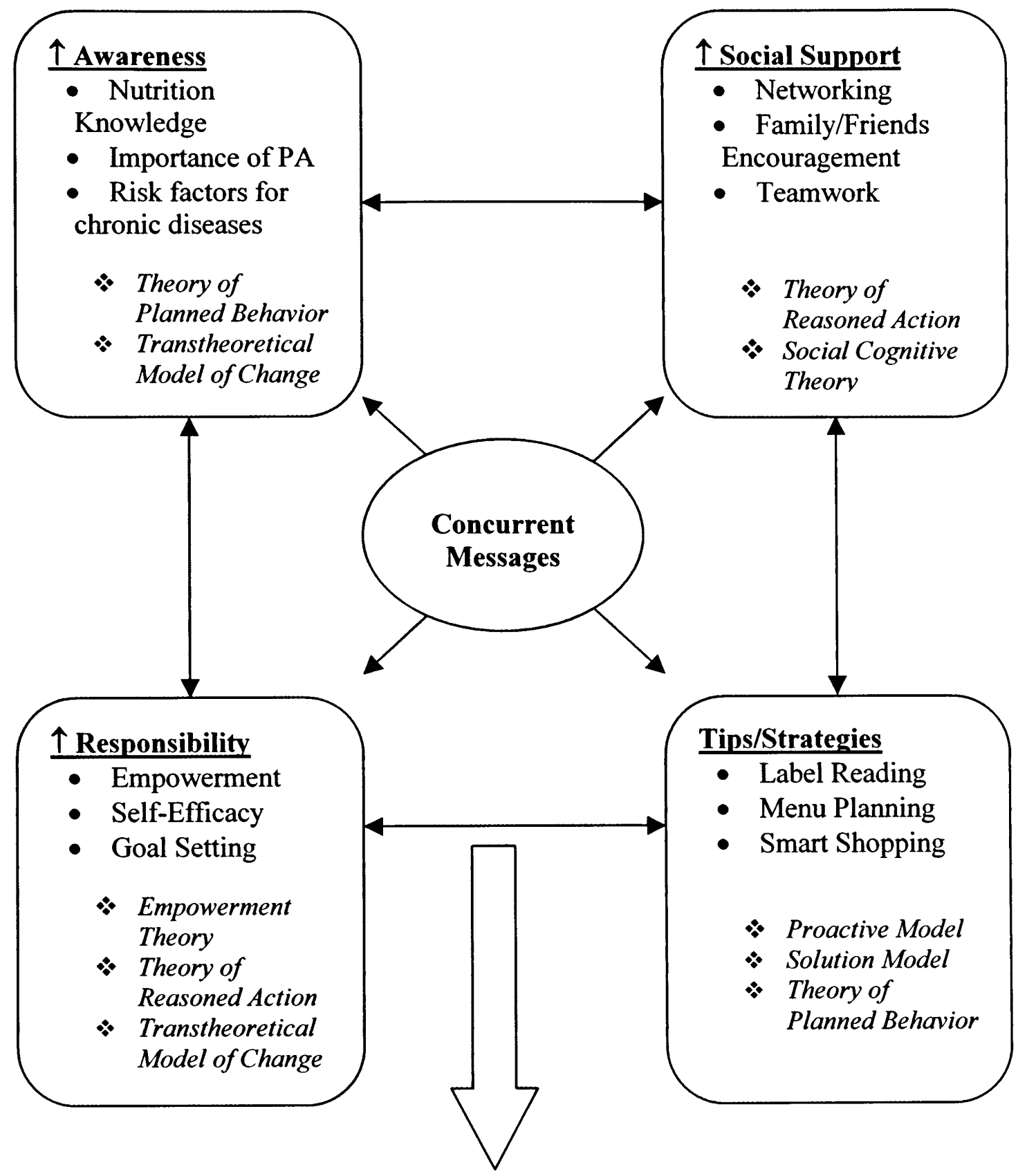

\section{Improved Dietary and Physical Activity}

Patterns

Risk Factors for Chronic Disease 
easy to prepare, and tips on shopping. Another important aspect of the practical approach was social support. A social component addressed social support since it can have a major impact on outcomes as well (Sallis et al, 2000). Participants were encouraged to network with others, join or form activity clubs. People who make healthy lifestyle changes often have help (Kirn, 2000; Berry 2003). Additionally, encouraging the participants to take responsibility and goal setting for improved health was stressed. The behavioral component focused specifically on the change process, skill building, incentives, and readiness (Hoelsher, 2000; Lytle, 2000; Contento, 1995). This component attempted to tie all of the other components together so the participants could use what they learned on a daily basis to improve their lifestyle choices.

The intervention groups received the same information content and both groups included the same components. Both the $\mathrm{CBI}$ and TDI interventions provided students the opportunity to learn about food and health, develop skills necessary to apply the information and incorporate these newly learned skills into everyday life (See Lesson Plans- Appendix B). The difference between the CBI group and TDI group was only in the method of delivery.

In order to be practical, information has to be disseminated in a relevant, understandable way. Adolescents in a school setting are quite used to the traditional didactic delivery of information. Nevertheless, research indicates that the learning styles of adolescents' in the $21^{\text {st }}$ century have evolved such that "teaching by telling" may no longer be the most effective method of eliciting a behavior change and delivering the information in a way that is entertaining, animated, and interactive may play a major role on outcome behaviors (Jensen, 2000). Additionally, students could send emails and get 
feedback within 24-hours regarding their lifestyle choices. Emails also could be used to enhance communication between one another, enhancing the social support network. The $\mathrm{CBI}$ group was given an email address in which they could submit questions to the principal investigator (PI) regarding lifestyle behaviors. The PI answered the emails within 24 hours.

\section{$\underline{\text { CD-ROM }}$}

It is estimated that between $80-90 \%$ of what we learn is visual (Jensen, 2000). Our brain gives priority to color, light, movement, form, and depth. The combination of these factors often cannot be attained using traditional methods of teaching. On the other hand, computer-assisted dissemination of information has the potential to more accurately reflect the brain's ability to process information. The CD-ROM developed for this study presented information using color, light, and animations. The brain has an immediate and basic response to symbols, icons, and other simple images (Jensen, 2000). Therefore, computer-assisted instruction has the ability to improve the learning environment by offering a variety of information mediums with an array of colors and pictures that also incorporate motion.

\section{Preliminary Quantitative Study Used to Test in Similar Population Before Initiation}

\section{of Research Study}

A preliminary quantitative study ( $\mathrm{n}=93$ ), approved by the Institutional Review Board at FIU and the Research and Assessment Review Board of the School Board of Broward County was conducted for two purposes. One purpose was to ascertain the feasibility and usability of an abbreviated version of the CD-ROM as well as several of the tools to be used in the study (the nutrition knowledge, physical activity, social 
support, the self-efficacy questionnaires). The other was to provide a foundation for the hypothesis that a computer based health education program would elicit greater improvement in outcome measures over the traditional "teaching by telling" method.

A high school in southern Broward County was chosen as the site for the pilot study (Table 1). A school with a diverse student population similar to those that would be used in the main study was purposefully chosen to be used in the intervention. The study took place over a 3-week period. Student volunteers were assigned to three groups. Group one $(n=36)$ served as a control $(\mathrm{CON})$. Subjects in this group did not receive any health education. Group two $(n=26)$ received information via the traditional method (TDI) including lecture, pamphlets, and brochures. Group three $(n=31)$ received the computer-based intervention (CBI), used a CD-ROM containing one module that summarized the 5 sessions to be used during the main study.

During week one, subjects in all three groups completed the nutrition knowledge, physical activity, self-efficacy, and social support questionnaires. During week 2 the CBI group used the CD-ROM and navigated their way through a 45-minute interactive health education module emphasizing the importance of a healthy diet and physical activity and strategies for maintaining a healthy weight. The CD-ROM information was used as a template for the design of the traditional didactic intervention method (TDI). During week 2 , the TDI group was given a 45-minute presentation accompanied by pamphlets and brochures emphasizing the importance of a healthy diet and physical activity, and strategies maintaining a healthy weight. During week 3 , all participants again completed the nutrition knowledge, physical activity, self-efficacy, and social support questionnaires. 
Table 1

Sociodemographic Data from School Used in Preliminary Studies

\begin{tabular}{|c|c|c|}
\hline Total Enrollment & & 3172 \\
\hline \multicolumn{3}{|l|}{ Ethnicity } \\
\hline & White, non-Hispanic & $31 \%(998)$ \\
\hline & Black, non-Hispanic & $23 \%(740)$ \\
\hline & Hispanic & $38 \%(1204)$ \\
\hline & Asian & $5 \%(152)$ \\
\hline & Multiracial & $2 \%(76)$ \\
\hline & Other & $0 \%(2)$ \\
\hline \multicolumn{3}{|l|}{ Gender } \\
\hline & \multirow{2}{*}{\multicolumn{2}{|c|}{$\begin{aligned} \text { Males } & 52.6 \%(1667) \\
\text { Females } & 47.4 \%(1505)\end{aligned}$}} \\
\hline & & \\
\hline Minority Rate & \multicolumn{2}{|r|}{$67 \%$} \\
\hline Free/Reduced Lunch & \multicolumn{2}{|r|}{$27 \%$} \\
\hline \multicolumn{3}{|l|}{ School Grade } \\
\hline (assigned according to & 2001 & $\mathrm{C}$ \\
\hline FCAT scores, school & 2002 & $\mathrm{C}$ \\
\hline \multirow[t]{2}{*}{ attendance, etc) } & 2003 & $\mathrm{C}$ \\
\hline & 2004 & D \\
\hline
\end{tabular}

The information presented to the CBI and TDI groups was tailored specifically to the needs of adolescents. The constructs of this practical approach (responsibility, communication, leadership, budgeting, and time management) were incorporated into the intervention and were presented on a level specifically targeted to this population. This 
approach has the potential to teach adolescents the skills needed for addressing health issues and taking responsibility for their own health.

After one session, results indicated a trend toward improvement in nutrition knowledge, self-efficacy for dietary change, and social support for both the CBI and TDI groups compared to the CON group. The CBI group tended toward greater improvements than the TDI group. However, these results were not significant.

\section{$\underline{\text { Research Study }}$}

\section{$\underline{\text { Intervention }}$}

The behaviors emphasized in the research study were increased daily physical activity, improved dietary habits (emphasizing a decrease in total fat, an increase in fruits and vegetables, fiber intake, and an increase in low-fat dairy intake), increased occurrence of nutrition label reading, decreased number of meals skipped, increased perceived social support score, and increased perceived self-efficacy score. This intervention included components of comprehension and application of information that is included in both the science and health (nutrition, physical activity, chronic diseases) benchmarks tested by the FCAT. During the intervention, all subjects were taught behavioral skills such as menu planning and smart shopping tips, so that participants could apply the knowledge and skills learned throughout the program. Although this was not specifically geared toward FCAT, the inclusion of this type of educational material made participation more desirable for principals and teachers. In addition, various incentives were offered to enhance student participation and encourage healthy behaviors such as pedometers, coupons and samples, portable radios, school supplies, and T-shirts. 
The health education was delivered to the CBI group via a CD-ROM designed by the principal investigator. Several nutrition educators and secondary education specialists reviewed the lessons for construct and content validity. Additionally, high school adolescents and parents evaluated the modules for usability, feasibility, and ageappropriateness. All evaluations were positive and few suggestions for improvements were made. The $\mathrm{CD}$ was edited and then re-evaluated. No further suggestions for improvement were made at that time.

Although the principal investigator (PI) was present to offer technical assistance in regard to the operation of the hardware and software, students individually followed instructions and prompts on the CD-ROM to navigate through the program. The students were each given a business card with an address to email any questions to the PI at any time during the intervention. A study guide with an answer key accompanied the navigation (Appendix B).

The CD-ROM information served as the template for the development of the traditional education. The same information was presented using overheads, brochures, and pamphlets in lecture/discussion form delivered by the PI. The education materials were designed based on the template used for the CBI materials to ensure that both intervention groups received the same content. Like the CBI version, the TDI version included five 45 -minute sessions. These sessions were lectures delivered by the principal investigator accompanied by hand-outs (pamphlets and brochures). The PI facilitated and encouraged questions that arose during each session. The TDI group also received the $\mathrm{e}$ same non-graded study guide (with answers) the CBI group received. The research intervention used included 5 sessions over the course of one week, each lasting 
approximately 45 minutes. Each lesson was offered 4 times during the day to accommodate the students' schedules. The content (Appendix B) of the behaviorally focused health education sessions included (1) Introduction to Adolescent Nutrition: Dietary Guidelines, the Food Guide Pyramid, and the Importance of Physical Activity; (2) Overview of the Nutrients [carbohydrate, protein, fats, vitamins, mineral, and water]; (3) Nutrition for Life; the Role of Nutrition and Physical Activity in preventing related to Chronic Disease (obesity, heart disease, and type 2 diabetes) (4) Taking Responsibility for Your Health: Setting Goals, Building Support, Improving Self-efficacy; and (5) Menu Planning, Smart Shopping, and Nutrition Labels. Although these topics were the primary focus, subject matter from previous sessions was re-emphasized throughout. For example, goal setting was touched upon in each session and the relationship between a healthy lifestyle and disease prevention was a recurrent theme. Lessons for the CBI group were delivered using the CD-ROM developed by the PI.

To decrease attrition, designated make-up sessions were available for students who missed one intervention session or data collection day. These sessions were offered by appointment only and scheduled throughout the intervention, as well as at the end of the intervention.

\section{Recruitment of Schools}

Florida International University (FIU) Institutional Review Board (IRB) and the

School Board of Broward County (Florida) Department of Research and Evaluation approved the research protocol. There are several challenges encountered when attempting to implement an intervention within the public school system. Schools have their own agendas and there are a number of different stakeholders. The Florida 
Comprehensive Assessment Test (FCAT) is used to assess student achievement statewide and has a huge impact on the curriculum and access to students for research purposes is limited. Therefore, the decision for a school to participate in a research study was made with great consideration. A number of schools were contacted and although several schools expressed interest, many were limited by scheduling conflicts or prior commitments. Three ethnically diverse schools in a similar geographically location offered letters of support and agreed to participate. Care was taken as not to disrupt the curriculum or school schedules; curriculum mandates and testing schedules were also considered. The three schools were ethnically diverse and enrollment was $\geq 70 \%$ minority students (Table 2). All students enrolled in the selected schools, which signed an informed consent and obtained parental consent, and met the inclusion criteria, were eligible to participate in the study.

\section{Recruitment of Students}

As core classes are essential to maintaining the rigid standards set forth by the FCAT, only students enrolled in at least one elective class were recruited for the study. Information did, however, coincide with FCAT learning skills and was based on standards of health education standards set by the State of Florida.

An "awareness campaign" was initiated at the end of the 2003-2004 school year. The purpose of this campaign was to introduce the program and to advertise it beginning at the start of the next school year (2004-2005). Actual recruitment occurred in the Fall of 2004 with the use of flyers, class announcements, and the Principal Investigator (PI) and assistants talking to students outside the cafeteria one or two days per week for the duration of the recruitment period (Appendix A). Students were offered information 
regarding the program. If they were interested in participating parental consent forms and teacher permission forms were distributed to interested students.

Table 2

Study Schools Ethnic, Gender, FCAT and Free Lunch Breakdown (2003-2004)

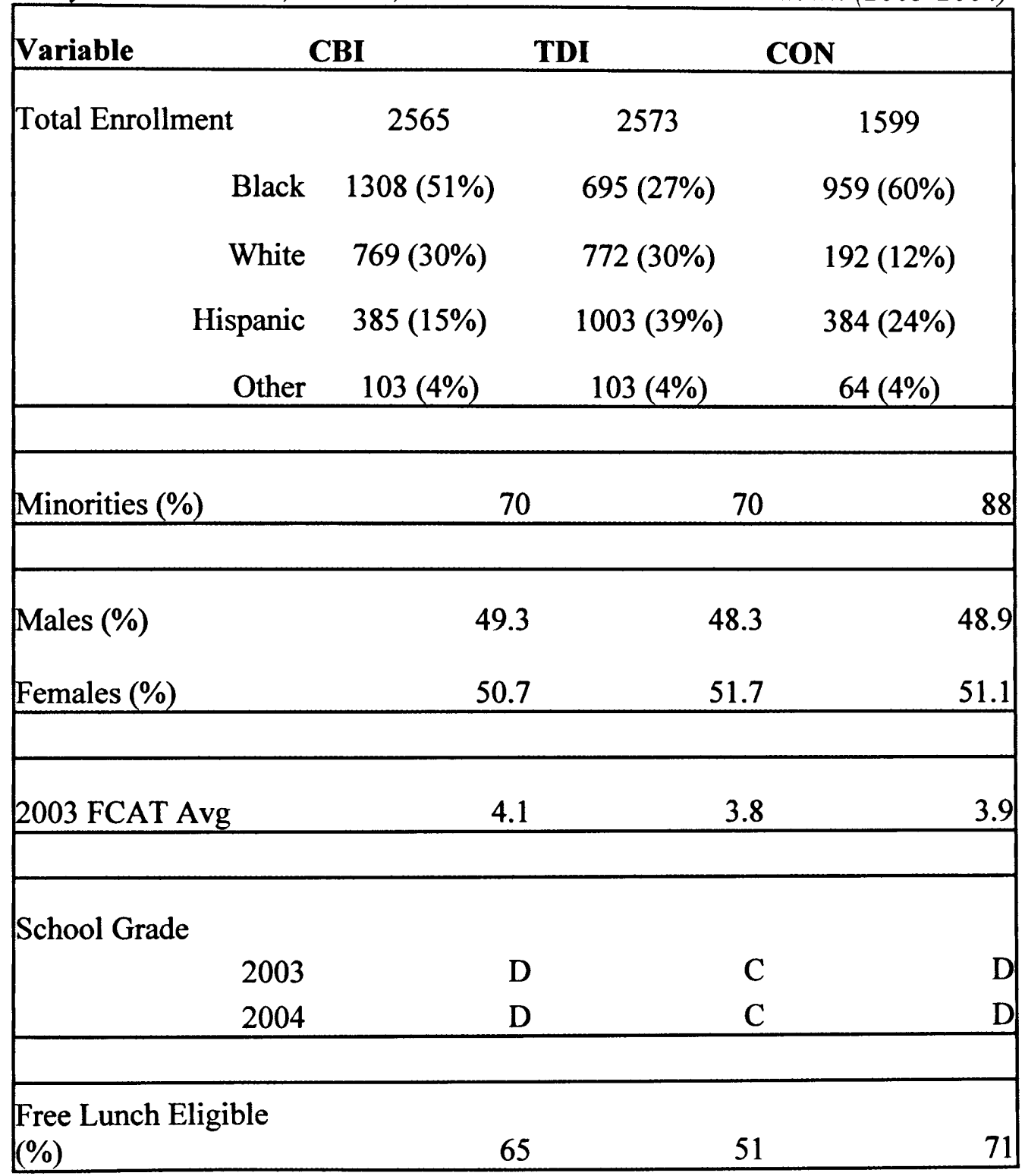




\section{Screening}

When recruitment was completed, screening of the subjects who volunteered began. The principal investigator determined eligibility and set appointments for the start-up session (baseline measurements). Only those who returned their signed parental consent forms and teacher permission forms were eligible to continue in the screening process.

\section{Inclusion/Exclusion Criteria}

\section{Inclusion Criteria}

1) Student enrolled in one of the three selected schools

2) Age 13-19 years

3) Parental consent obtained

4) Completion of informed consent by student

5) Teacher permission obtained

6) Commitment to participate in an intervention program of 6 months duration (approximately 12 meetings)

\section{Exclusion Criteria}

1) Prior or current participation in another health education program

2) Students under the care of a physician or not in good health

3) Participants unable to speak and/or write in English

4) Students not willing to meet commitments outlined in inclusion criteria

During the screening session, both the parental consent and student informed consent forms (Appendix F) were thoroughly explained to each participant. Participants had the opportunity to ask questions regarding the study. Signing the student informed consent indicated the participant's commitment to participate. Subjects were told that participation in the study was in no way correlated with academic requirements and they could withdraw from the study at any time. Participants were asked to provide their class schedule and were then given a teacher permission slip. Teacher permission had to be 
obtained to miss a class. An "in-school activity" permission slip that is commonly used within the school system was used for this purpose.

Once students were screened and deemed eligible to participate, appointments were set based on their schedule availability (i.e. during elective course offerings). At that time, the day of the week and the class period was designated for the intervention activities. Actual meeting days were assigned before the intervention began.

\section{Study Design}

Three racially/ethnically diverse schools were selected within Broward County. They were randomly assigned to one of three groups as follows: One school served as the control school (CON), one school as the traditional didactic instruction school (TDI), and the third as the computer based intervention school (CBI). Assigning schools rather than individual students to a treatment group was done to eliminate the possibility of intergroup communication within the same school. However, this design does not control for factors or experiences that may be unique to each school. The limitations of the design decision are thoroughly discussed in limitations section of discussion.

For a one-way repeated measures ANOVA, the sample size needed to detect a significant difference between the three groups was calculated to be $n=159$ participants. Equal sample sizes of 53 would result in $80 \%$ power to detect a medium effect size (.25) using an alpha equal to .05 (Cohen, 1988). Allowing for a $33 \%$ dropout rate, at least 80 participants per group were recruited.

\section{Baseline Measures}

There were two 45-minute sessions during which baseline data were collected (Appendix A). The same protocol was used for all groups at all data collections. In the 
first baseline session, the PI and trained assistants measured the weight and height of each participant. Participants were asked to complete the nutrition knowledge, and physical activity questionnaires. An interview was conducted to obtain the first 24-hour recall. During the second baseline data collection session, the second 24-hour dietary recall was obtained and participants completed the social support, dietary and eating confidence, and food frequency questionnaires.

\section{Outcome Measurement Timetable}

Outcome measures were assessed at two points: post-intervention measures were performed immediately after the last intervention session (approximately $2 \frac{1}{2}$ months after baseline measurement) and follow up measures were taken approximately 14 weeks after post-intervention outcome measures (approximately 6 months after baseline). The outcomes measured included nutrition knowledge, physical activity, food frequency, social support, self-efficacy, and readiness questionnaires, anthropometric measurements, and dietary interview using two non-consecutive 24-hour recalls.

Subjects in the (CON) group did not receive the intervention, but completed the baseline, post, and follow-up data collection sessions: anthropometric measurements (height, weight), physical activity questionnaire, nutrition knowledge test, social support survey, eating confidence survey, and two non-consecutive 24-hour recalls (per data collection). In an effort to provide the same benefit to all participants, following completion of the study, the CON group received similar information (pamphlets and textual information) as that received by the TDI group and also received any participation incentives offered to the other groups. 


\section{Instruments (Appendix C)}

All participants completed baseline, post, and follow-up measures including: anthropometric measurements, two non-consecutive 24-hour recalls, food frequency questionnaire, a nutrition knowledge questionnaire, physical activity questionnaire, dieting and exercise confidence survey, and social support questionnaire.

\section{Anthropometric Measurement}

\section{Height and Weight}

Body Mass Index (BMI) is calculated using height and weight measurements. There are well- established limitations for using BMI calculations for adolescents usually due to complications by varying growth rates and maturity levels (Chumlea et al, 2001). Nevertheless, BMI has been reported to be used effectively to monitor changes in body mass, as comparisons can be tracked over time and perform well as a diagnostic test for fatness (Neovius et al, 2005). Furthermore, Wang (2004) concluded that BMI is most likely the best choice of obesity assessment among available measures. The CDC has deemed the use of BMI in adolescents to be the recommended screening assessment (CDC, 2004).

Participants' height was measured after removal of shoes using a metric measuring tape affixed on the wall. Weight was measured on a digital scale. The scale used was a digital lithium scale model named Health-o-Meter ${ }^{\circledR}$ manufactured by Sunbeam Products, Inc. in Boca Raton, FL. The same scale was used at all three sites and was placed on a bare floor at each site. It was calibrated with 10-pound calibration weights before each use. Each participant was weighed twice and the two readings were averaged to obtain one value. Participants were weighed with light clothing. BMI was 
determined by the following formula: BMI $\left.=[\text { weight (lbs.) } / \text { height (in) })^{2\}}\right] \times 703$ (Krause, 2002).

\section{$\underline{\text { 24-Hour Recall }}$}

The 24-hour recall is the most widely used method to assess nutrient intake in population studies (Monsen, 1992). There are both strengths and limitations to this tool. The 24-hour recall can be administered relatively quickly and data may be entered directly into a dietary analysis program to assess a participant's diet quality. Because of the ease of use and lack of focus on literacy, it is appropriate for most people including children and adolescents (Stang, 2002). However, like many dietary intake tools, it relies on memory and self-reported information. Although the 24-hour recall is considered reliable in terms of mean nutrient intakes for populations because of daily variance in food intake, serial 24-hour recalls are recommended (Stang, 2002; Monsen, 1992).

The CFSII as well as several other large-scale dietary assessment surveys have utilized the multiple pass method for increasing accuracy of dietary recalls (Johnson et al, 1996). Using this method the participant is asked to recall all foods and beverages consumed during the past 24 hours and food models are used to estimate serving size. On the second pass more probing questions are asked in an effort to gain additional information or gather information on any food or beverage that may have been overlooked. On the third pass, the recall is read back to the participant and serving sizes double-checked to improve accuracy and again gather information on any item that may have been disregarded (Johnson et al, 1996).

As was done in the CFSII, the multiple pass method was used (Figure 3) (USDA, 2004). Participants were asked to recall all foods and drinks consumed during the 
Figure 3. Multiple-Pass Method*

\section{Interview \\ 5 Steps}

\section{Purpose}

Step1

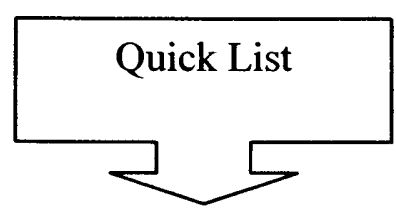

Collect a list of foods consumed the previous day

Step 2

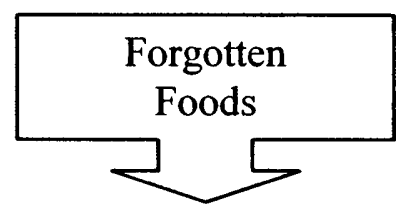

Collect foods that may have been forgotten during the quick list. Questions probe for foods by categories: beverages, candies \& sweets, snacks, etc.

Step 3

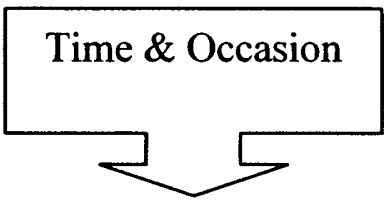

Collect time and name of eating occasion for each food

Step 4

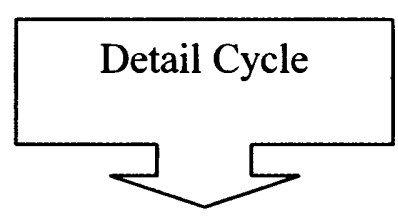

Collect detailed descriptions of each food, including amounts and additions to food. Also, summarize foods at eating occasion and probe times between occasions for forgotten foods.

Step 5

Final Probe

Collect additional foods not remembered earlier

*USDA, Agricultural Research Service, Food Surveys Research Group 
previous 24 hours. Verbal probing was used to elicit more information when responses were vague. Students were also asked to think of anything else they may have consumed but did not mention at the close of the interview. The 24 -hour recall was administered in an interview setting for 2 randomly assigned, but non-consecutive days within a twoweek period at each data collection. The 24-hour period was defined and used consistently. The 24-hour period ended with intake before arriving at school the day of the data collection and 24-hours earlier. Due to scheduling, it was not possible to include a weekday and a weekend day for each participant, so only weekdays were used. As a result, 24-hour recalls were not collected on Mondays. Interviews also included questions on quantification, which were standardized with food models. Whenever possible, brand names, culture-specific foods, method of preparation, and ingredients were recorded.

The 24-hour recalls were analyzed using the NutritionCalcPlus ${ }^{\circledR}$ (USDA) database. Individual foods were entered by brand name and portion size into the database for each participant. Foods that were not in the database were researched for ingredients and added to the database so that analysis of those foods could be done. However, this was rarely an issue. Two dietary recalls were analyzed together and mean/average for a data collection time point was computed. Data analyzed were calories, fat (and saturated fat), fruit and vegetable, and dairy intakes.

Food Frequency Questionnaire

The Food Frequency Questionnaires (FFQ) is a valid and reliable tool that can be utilized to determine the foods currently consumed as well of those consumed in the recent past (Monsen, 1992). Although it does not provide valid estimates of absolute 
intake for individuals (Stang, 2002), it can be used to provide information about the types of foods consumed by the target population over a period of time and can be compared to responses obtained through 24-hour recalls (Buzzard et al, 2000). The FFQ was used to compare to the results of the 24-hour recalls for further assessment of diet quality.

The participants also completed a FFQ, which assessed only fat, fruit and vegetable, and fiber intake. The FFQ used was the Goals for Health Questionnaire (Buzzard et al, 2001). The Goals for Health Questionnaire is a shortened version of the widely used Youth/Adolescent Questionnaire (YAQ), which was developed for ethnically diverse populations of adolescents (Buzzard et al, 2001). The original YAQ is a 151-item questionnaire, but because of the need for brevity the short version was selected. The short version of this questionnaire has been validated and evaluated for reproducibility (Buzzard et al, 2001). In general the correlation coefficients for repeat administrations of FFQ among adolescents tend to be low. This questionnaire is fair in comparison to others including the original YAQ with correlation coefficients 0.24 to 0.59 with a mean correlation of 0.41 (Buzzard et al, 2001). The YAQ reported correlation coefficients of 0.26 to 0.58 with a mean correlation of 0.42 (Perks et al, 2000).

The last question on the questionnaire was used to determine the label reading habits of subjects. Using a 5-item Likert-type scale students were asked to indicate their frequency of label reading with choices ranging from " $1=$ almost never" to " $5=$ almost always." 


\section{Other Assessments}

\section{Nutrition Knowledge Questionnaire}

The questionnaire developed by Parmenter and Wardle (1998) contains 116 items regarding thoughts about advice experts give, classification of foods into food groups, selection of healthy food choices, correlations between diet and disease. An additional 7 items are used to determine demographic data for participants. According to the authors, it has the potential to be utilized to identify weaknesses in a person's understanding of nutrition and healthy eating as well as provide data for examining the relationship between knowledge and dietary behaviors (Parmenter and Wardle, 1998). After being tested for reliability and construct validity among the population for which it was designed it was found that the overall reliability was 0.98 and that of individual subsections reliabilities were also very high ranging from 0.8 to 0.97 (Parmenter and Wardle, 1998). In addition nutrition experts scored significantly better than experts in other fields indicating good construct validity.

The nutrition knowledge questionnaire designed and validated by Parmenter and Wardle (1999) was developed for the "general public" in the United Kingdom (adolescents to adults up to the age of 75) to provide a reliable and valid tool to measure nutrition knowledge. In this study the tool was used unchanged except for the 4 items where equitable foods or food groups were substituted for similar American foods. For example, beans on brown rice was substituted for beans on whole-wheat toast, oatmeal was substituted for porridge, omelet for quiche, and ham for gammon. The structure and content was otherwise left intact. 
Items 1-10 measured knowledge that advice experts give, 11-38 measured knowledge of food groups, 39-106 measured knowledge of healthy food choices, and items 107-116 measured knowledge of relationship of the diet to chronic disease. The last seven questions were used to determine the demographic data regarding age, gender, parents' education, ethnic origin, grade level, employment status, and current dieting practices.

\section{Physical Activity Questionnaire (PAQ-a)}

Studies indicate that a 7-day recall of physical activity is sufficient for monitoring physical activity patterns in children and adolescents (Sallis, 2002). The PAQ-a is a 9question assessment that has been specifically designed for use with adolescents. Three studies were completed to assess the reliability and validity specifically for the PAQ-a (Crocker, 1997). Each study indicated that the PAQ-a had acceptable item distribution, corrected item total correlations, and internal consistency. The test-retest reliability was also acceptable for both males and females across the age group of 8-14 years. Increasing age is associated with even higher scores of reliability for physical activity recalls and is highest with use of subjects 14 years old or older (Sallis et al, 1993). Since its development, the PAQ-a has been used in numerous studies with adolescent populations. The ages of the selected participants in this study fall into the category associated with the highest scores of reliability. Additionally, generalizability coefficients exceeded 0.80 for the population studied.

A modified version of the validated International Physical Activity Questionnaire (IPAQ), called the PAC-a, was used to estimate average daily physical activity (Crocker et al, 1997). The PAQ-a, designed specifically for adolescents, was developed to assess 
overall level of physical activity throughout the school year for students beyond grade 8 (ages 14-20). It is a guided self-administered questionnaire that asks students to recall physical activities for the previous 7 days. A strong correlation $(r=0.62, P<0.001)$ has been reported between actual physical activity and reported physical activity in adolescents (Crocker et al, 1997). Designed to be used in studies with large samples of school-aged children and adolescents, it can be administered in a classroom setting and takes approximately 10-20 minutes with minimal guidance. It consists of nine items including information on daily leisure time activity, in-school physical education, lunchtime physical activity, evening and weekend activity, and a self-rating physical activity scale (Kowalski et al, 1997). The total score can be converted to an activity level ranging from sedentary to vigorously active. There is a numerical range assigned to each level.

The PAQ-a was produced in the northern United States, and some of the winter activities listed differ greatly from activities participated in by South Florida adolescents (i.e., snow skiing). As a result some substitutions were made to reflect more common activities of adolescents in southern States. The format remained the same. Changes made included the addition of basketball, the deletion of cross-country skiing, and the substitution of racquetball/handball for squash.

Dietary and Exercise Confidence Survey

There appears to be a mutual relationship between personal factors such as selfefficacy, environmental factors, including perceived barriers, and health related behaviors such as diet and physical activity. The Dietary and Exercise Confidence Survey is a 32item survey developed to measure the degree to which subjects are sure they can make 
dietary and exercise behavior changes despite the aforementioned factors (Sallis et al, 1988). Internal consistency reliability coefficients range from 0.83 to 0.85 . Additionally, test-retest reliability for this instrument has been determined to be adequate (Sallis et al, 1988). Since its development in 1988 , it has been used as a valid tool in many other studies including the Patient-centered Assessment and Counseling for Exercise plus Nutrition (PACE+) (Sallis et al, 2000), Graduate Ready for Activity Daily (GRAD) (Sallis et al, 1997), and Project WALK Exercise and Health Study (Chen et al, 1998). It has been modified and used in a number of other studies, as well (Dwyer et al, 1998).

The affective and behavioral components of the approach used in this intervention incorporated a self-efficacy construct to enhance behavior change. Eliciting a change in dietary and exercise habits is difficult to initiate and many past interventions have resulted in little, if any, change in behavior. Self-efficacy has been determined to be a strong mediator of behavior change. The Dietary and Exercise Confidence Survey, developed by Sallis et al (1988) is a two-part survey that measures self-efficacy for exercise and eating behaviors. The exercise behavior portion has two factors, resisting relapse and making time for exercise. The test-retest reliability coefficient for both factors has been determined to be 0.68 and the internal consistency was calculated at 0.85 and 0.83 respectively (Sallis et al, 1988). The eating behaviors portion has 5 factors: resisting relapse, reducing calories, reducing salt, reducing fat, and behavioral skills. The test-retest reliability for these five factors ranged from 0.43 to 0.64 while the internal consistency ranged from 0.85 to 0.93 . Since its development in 1988 , the tool has been used in large-scale studies with diverse populations of adolescents including the PACE+, 
GRAD, and Project Walk interventions (Sallis, 2004). Additionally, the self-efficacy reliability (Cronbach's alpha score) was reported to be 0.70 (Sallis et al, 2004). Social Support Survey

Parent and peer support is significantly correlated with adolescent health behaviors (Prochaska et al, 2002). The Social Support Survey is a 23-question assessment that was developed to measure perceived social support specific to healthrelated eating and exercise behaviors (Sallis et al, 1987). In developing this instrument, specific supportive and non-supportive behaviors were first identified through interviews with individuals attempting to make dietary and exercise behavior change. These items were then formulated into a survey and administered to 171 adults and adolescents. Statistical analysis determined both the test-retest reliability and internal consistency reliability to be satisfactory and significant evidence of criterion-related validity was noted (Sallis et al, 1987). Since its development in 1987, it has been used as a valid tool in many studies including the Patient-centered Assessment and Counseling for Exercise plus Nutrition (PACE+), Graduate Ready for Activity Daily (GRAD), and Project WALK Exercise and Health Study (Sallis et al, 2004; Chen et al, 1998).

A number of factors play a role in the lifestyle behaviors of adolescents. The older the adolescent, the more independence they have from their parents for making their own food choices. However, social norms and peer pressure play a major contributory role in these choices and families retain at least a limited influence. Therefore addressing the issue of social support appears to be an important aspect of behavior modification. As part of the affective arm of the intervention, participants were encouraged to empower themselves to make healthy decisions and to garner the support 
of their family and friends. The Social Support Survey developed by Sallis et al (1987) was designed to assess the perceived social barriers set forth by family and friends that one may encounter when trying to improve eating habits or to exercise regularly. Using a 5-point Likert scale the survey asks participants to rate the level of encouragement received from family or friends toward an attempted behavior modification ( $1=$ rarely ever encourages, $5=$ almost always encourages).

\section{Incentives}

Tangible and intangible incentives and rewards are often used as motivators to keep children and adolescents interested in learning. Not only are they a useful technique to keep participants compliant to study protocol, but reinforcements can also serve to prolong healthful behaviors and be used as reinforcement of a message (JADA, 1999). At each intervention participants were given a small incentive. Incentives were chosen to coincide with particular lessons. Incentives included Frisbees, magnets, cups, radios, pedometers, backpacks and others. There was also a poster contest with a cash prize that all participants were eligible to enter (Appendix C). The theme of the poster contest was "Nutrition as it Relates to Chronic Disease."

\section{Confidentiality of Data}

Confidentiality of the subject's identity was maintained by recording all of the data using a personal identification number (PIN) only. This number was used as the only identifier for all participants' survey instruments. All written subject keys containing personal information or associating names and PIN were kept in a locked file cabinet of the PI. 


\section{Statistical Analysis}

\section{Descriptive Statistics}

Descriptive statistics including means, standard deviations, frequencies and percentages (using SPSS, version 11.0) were used to summarize socio-demographic data, dietary intake variables, nutrition knowledge, participation in physical activity, perceived self- efficacy, and perceived social support.

Repeated Measures ANOVAs

A repeated measures model was chosen for statistical analysis of outcome measures (dietary intake, nutrition knowledge, BMI, physical activity, social support, self-efficacy, and label reading) since each participant was measured at three points in time: baseline, post, and follow-up on each outcome measure. The intervention program type (computer based, traditional didactic and control) was the between subjects factor. The following illustrates the design and sample sizes used in this repeated measures ANOVA approach.

Figure 4. Repeated Measures ANOVA Approach.

\begin{tabular}{|c|l|l|l|l|}
\hline $\begin{array}{l}\text { School } \\
\text { (Treatment) }\end{array}$ & Baseline & Post & Follow-Up & $\mathbf{n}$ \\
\hline TDI & 87 & 87 & 87 & 87 \\
\hline CBI & 84 & 84 & 84 & 84 \\
\hline Con & 83 & 83 & 83 & 83 \\
\hline $\mathrm{n}$ & 254 & 254 & 254 & \\
\hline
\end{tabular}

The model tested for both main effects of group and time and for the group by time interaction. Post hoc tests (Bonferroni) were used to test for pair-wise differences 
for significant interactions or main effects. Tests were declared statistically significant if $\mathrm{p}<0.05$.

From the 24-hour recalls, counts were obtained on the frequency of meals skipped, lunch meals purchased from a vending machine, and number of times the participant chose foods from the "pizza line" for lunch. Because these data were counts, square root transformations were performed to normalize data before analysis using repeated measures ANOVA.

Preliminary analysis of the three treatment groups indicated there were no major differences in the primary outcome measures at baseline. Participants in all three groups were found to be comparable in nutrition knowledge, BMI, physical activity, and dietary behaviors at baseline.

Analysis of Covariates

To control for the variance that may be associated with pre-existing individual differences among the subjects, multiple analyses of covariance (ANCOVAs) were performed. These ANCOVAs were used to determine whether any of the factors of age, gender or ethnicity affected the results. ANCOVAs were performed on nutrition knowledge, BMI, physical activity, and dietary intake. No significant covariates were found so were not used in the further analyses.

\section{Analysis of Questionnaire Responses}

For the nutrition knowledge questionnaire, both the percent of correct answers in the nutrition knowledge questionnaire and the individual scores in each of the nutrition knowledge areas were analyzed. For each question, there was only one correct answer and scores were based on the number of correct answers of each participant. 
Total scores were calculated using the instructions set forth by the authors of the each of the other questionnaires. In the PAQ-a, there were 9 questions regarding typical physical activity. A total physical activity score was determined for each participant. Based on that score, a rubric was used to define the activity level.

Self-efficacy scores were totaled for six different areas; four for dietary confidence and two for exercise. The questionnaire was scored so that each subset was grouped and an average was determined for behaviors relating to that particular subsection. Social support scores were totaled for family and friends and separated by encouraging behaviors and discouraging behaviors. The FFQ specifically focused on fat, fruit and vegetable, and fiber intakes. 
Figure 5. Methods Summary.

\begin{tabular}{|c|c|c|c|}
\hline Hypothesis & $\begin{array}{l}\text { Groups } \\
\text { Compared }\end{array}$ & Tool(s) & $\begin{array}{l}\text { Statistical } \\
\text { Analysis } \\
\text { Used }\end{array}$ \\
\hline $\begin{array}{l}\text { The CBI group will show a } \\
\text { greater increase in knowledge } \\
\text { compared to both the TDI and } \\
\text { control groups post-intervention } \\
\text { and at follow-up. }\end{array}$ & $\begin{array}{l}\text { CBI, TDI, } \\
\text { CON }\end{array}$ & $\begin{array}{l}\text { Nutrition } \\
\text { Knowledge } \\
\text { Questionnaire } \\
\text { (Parmenter \& } \\
\text { Wardle, 1997) } \\
\end{array}$ & $\begin{array}{l}\text { One-way } \\
\text { Repeated } \\
\text { Measures } \\
\text { ANOVA }\end{array}$ \\
\hline $\begin{array}{l}\text { The CBI group will greater } \\
\text { strides toward achieving a } \\
\text { healthy body mass index } \\
\text { compared to both the TDI and } \\
\text { control groups post-intervention } \\
\text { and at follow-up. }\end{array}$ & $\begin{array}{l}\text { CBI, TDI, } \\
\text { CON }\end{array}$ & $\frac{\mathrm{Wt}(\#)}{\mathrm{Ht}(\mathrm{in})^{2}} \times 703$ & $\begin{array}{l}\text { One-way } \\
\text { Repeated } \\
\text { Measures } \\
\text { ANOVA }\end{array}$ \\
\hline $\begin{array}{l}\text { The CBI group will show a } \\
\text { decrease in fat intake, especially } \\
\text { saturated fat, an increase in fruit, } \\
\text { vegetable, and fiber intake, and } \\
\text { increased consumption of low- } \\
\text { fat dairy products compared to } \\
\text { both the TDI and control groups } \\
\text { post-intervention and at follow- } \\
\text { up. }\end{array}$ & $\begin{array}{l}\text { CBI, TDI, } \\
\text { CON }\end{array}$ & $\begin{array}{l}\text { 24-Hour Recall } \\
\text { Food Frequency } \\
\text { Questionnaire } \\
\text { (Buzzard et al., } \\
\text { 2001) }\end{array}$ & $\begin{array}{l}\text { One-way } \\
\text { Repeated } \\
\text { Measures } \\
\text { ANOVA }\end{array}$ \\
\hline $\begin{array}{l}\text { The CBI group will show an } \\
\text { increase in physical activity level } \\
\text { compared to both the TDI and } \\
\text { control groups post-intervention } \\
\text { and at follow-up. }\end{array}$ & $\begin{array}{l}\text { CBI, TDI, } \\
\text { CON }\end{array}$ & $\begin{array}{l}\text { Physical Activity } \\
\text { Questionnaire for } \\
\text { Adolescents } \\
\text { (PAQ-a) (Crocker } \\
\text { et al, 1997) }\end{array}$ & $\begin{array}{l}\text { One-way } \\
\text { Repeated } \\
\text { Measures } \\
\text { ANOVA }\end{array}$ \\
\hline $\begin{array}{l}\text { The CBI group will show an } \\
\text { increase in frequency of label } \\
\text { reading compared to both the } \\
\text { TDI and control groups post- } \\
\text { intervention and at follow-up. }\end{array}$ & $\begin{array}{l}\text { CBI, TDI, } \\
\text { CON }\end{array}$ & $\begin{array}{l}\text { Survey Question } \\
\text { from FFQ }\end{array}$ & $\begin{array}{l}\text { One-way } \\
\text { Repeated } \\
\text { Measures } \\
\text { ANOVA } \\
\text { Cross-tabs }\end{array}$ \\
\hline $\begin{array}{l}\text { The CBI group will show an } \\
\text { increase in self-efficacy } \\
\text { regarding dietary and physical } \\
\text { activity habits compared to both } \\
\text { the TDI and control groups post- } \\
\text { intervention and at follow-up. }\end{array}$ & $\begin{array}{l}\text { CBI, TDI, } \\
\text { CON }\end{array}$ & $\begin{array}{l}\text { Diet and Physical } \\
\text { Activity } \\
\text { Confidence } \\
\text { Survey } \\
\text { (Sallis et al, 1988) }\end{array}$ & $\begin{array}{l}\text { One-way } \\
\text { Repeated } \\
\text { Measures } \\
\text { ANOVA }\end{array}$ \\
\hline $\begin{array}{l}\text { The CBI group will show an } \\
\text { increase in perceived social } \\
\text { support compared to both the } \\
\text { TDI and control groups post- } \\
\text { intervention and at follow-up. }\end{array}$ & $\begin{array}{l}\text { CBI, TDI, } \\
\text { CON }\end{array}$ & $\begin{array}{l}\text { Social Support } \\
\text { Survey } \\
\text { (Sallis et al, 1987) }\end{array}$ & $\begin{array}{l}\text { One-way } \\
\text { Repeated } \\
\text { Measures } \\
\text { ANOVA }\end{array}$ \\
\hline
\end{tabular}




\section{CHAPTER IV}

\section{RESULTS}

\section{Intervention Sample Characteristics}

Two hundred and seventy-five participants began the study. Data were analyzed only for participants who completed all three data collections and all 5-intervention sessions $(\mathrm{n}=254)$. Completers versus non-completers were statistically compared and no demographic differences were noted. Repeated measures ANOVA were used to analyze data. Table 3 provides frequencies, percentages, means, and standard deviations of participants' sociodemographic characteristics. Participants were primarily female $(65.8$ $\%)$, in grades 9-12 $($ mean $=10.55)$, and ranged in age from 13-18 years $($ mean $=15.79)$. Most were non-Hispanic black (53.1\%) with the second largest group being Hispanic (23.2\%). Approximately $30 \%$ were employed at the time of the study.

Differences noted among the groups at baseline included age, employment status, and parents' education. Students in the CBI group were employed at a significantly higher rate than students in the other two groups at pre and post $(\mathrm{p}<0.001)$ and fewer of their parents had completed high school compared to the parents of subjects in the CON and TDI groups $(\mathrm{p}<0.001)$. The CON group participants were younger than the CBI and TDI groups $(\mathrm{p}<0.01)$.

\section{Outcome Measures}

Hypothesis 1:

The CBI group will show a greater increase in knowledge compared to both the TDI and control groups post-intervention and at follow-up.

Data analysis revealed a significant time by treatment interaction of $(p<0.001)$ in overall nutrition knowledge (Table 4). There were no differences noted in nutrition knowledge scores at baseline among the groups, with an average across the groups of 
$41.7 \%$ correct responses. The CON group demonstrated no change in nutrition knowledge across time periods. However there was an increase in nutrition knowledge in both intervention groups ( $\mathrm{p}<0.001)$ from baseline to post and the CBI group' follow-up mean was still significantly higher than at baseline $(p<0.001)$. The higher scores for the $\mathrm{CBI}$ group at follow-up indicate that the $\mathrm{CBI}$ group was best able to retain the nutrition knowledge. At follow-up all three groups were statistically different $(\mathrm{p}<0.001)$ from one another, with the CBI group having the higher mean score $(54.7 \%)$ followed by the TDI group $($ mean $=48.3 \%)$, and the CON group $($ mean $=42.8 \%)$.

Nutrition knowledge scores were separated according to subject area (Table 5). There were no changes in any of the subject areas for the CON group over the course of the study. The interventions had no impact in the areas of experts' advice or food choices. However, there was a significant time by treatment interaction for knowledge about food groups ( $p<0.001)$ and knowledge relating diet to health $(p<0.001)$. The TDI and CBI groups both showed increases in knowledge about food groups from baseline to post $(p<0.001)$. The CBI group maintained the increase in knowledge regarding information on food groups at follow-up whereas the TDI group did not. The CBI group also showed an increase in knowledge relating diet to health $(p<0.001)$ at post and these increases were greater than the increases observed in the TDI group $(p<0.01)$. These differences were sustained by the CBI group through to follow-up such that the CBI group was different than both the TDI and CON group. 
Table 3

Demographics for Study Participants

\begin{tabular}{ccccccc}
\hline Ethnicity & \multicolumn{1}{c}{ CON } & CBI & TDI & Total & p-value \\
\hline & & $\mathrm{n}=87$ & $\mathrm{n}=84$ & $\mathrm{n}=83$ & $\mathrm{n}=254$ \\
& White & $6(6.9 \%)$ & $11(13.1 \%)$ & $19(22.9 \%)$ & $36(14.2 \%)$ & \\
& Black & $57(65.5 \%)$ & $55(65.5 \%)$ & $23(27.7 \%)$ & $135(53.1 \%)$ \\
& Hispanic & $16(18.4 \%)$ & $14(16.7 \%)$ & $29(34.9 \%)$ & $59(23.2 \%)$ & \\
Other & $8(9.2 \%)$ & $4(4.8 \%)$ & $12(14.5 \%)$ & $24(9.4 \%)$ & \\
\hline
\end{tabular}

Mean Grade $\quad 10.02^{\mathrm{a}} \quad 10.84^{\mathrm{b}} \quad 10.72^{\mathrm{b}}$ $10.52 \quad \mathrm{p}<0.01$

Mean Age (+/-SD) $15.4 \pm 1.1^{\mathrm{a}} \quad 15.9 \pm 1.1^{\mathrm{b}} \quad 15.9 \pm 1.1^{\mathrm{b}} \quad 15.8 \pm 1.1 \quad \mathrm{p}<0.01$

Employment Status

(\% Employed) Baseline

$15^{\mathrm{c}}$ $33^{\mathrm{d}^{* * *}}$

Post

$16^{\mathrm{c}}$

$36^{\mathrm{d}^{* * *}}$

$18^{\mathrm{c}}$

$66(26 \%) \quad \mathrm{p}<0.001$

Follow-Up

24

34

$24^{\mathrm{c}}$

$76(29.9 \%) \quad p<0.001$

$29 \quad 87(34.3 \%)$

Parent's Ed (>High School Ed)

\begin{tabular}{lllll} 
Mom & $42.5 \%^{\mathrm{c}}$ & $33.3 \%^{\mathrm{d}}$ & $48.2 \%^{\mathrm{c}}$ & $\mathrm{p}<0.001$ \\
Dad & $22.90 \%$ & $25.10 \%$ & $38.50 \%$ & \\
\hline
\end{tabular}

${ }^{\mathrm{a}-\mathrm{d}}$ Groups with different letters are statistically different, $\mathrm{p}<0.05$

$* * *$ Indicates increase from baseline to post, $p<0.001$

Table 4

The Effect of a Computer-based and a Didactic Intervention on Nutrition Knowledge Scores (\% correct) $)^{\wedge}$

\begin{tabular}{llll}
\hline & Baseline & Post & Follow-Up \\
\hline CON & $41.5 \pm 1.3$ & $43.2 \pm 1.2^{\mathrm{b}}$ & $42.8 \pm 1.1^{\mathrm{c}}$ \\
CBI & $40.7 \pm 1.3$ & $53.3 \pm 1.2^{\mathrm{a}^{* * *}}$ & $54.7 \pm 1.1^{\mathrm{d}^{* * *}}$ \\
TDI & $42.7 \pm 1.3$ & $50.9 \pm 1.2^{\mathrm{a}^{* * *}}$ & $48.3 \pm 1.1^{\mathrm{e}}$ \\
\hline
\end{tabular}

$\wedge$ Nutrition Knowledge Questionnaire developed by Parmenter \& Wardle (1999).

Values represented indicate mean $\pm \mathrm{SEM}$.

${ }^{\mathrm{a}-\mathrm{e}} \mathrm{Groups}$ in columns with different letters are statistically different, $\mathrm{p}<0.001$.

$* * *$ Indicates increase from baseline to post and/or baseline to follow-up, $\mathrm{p}<0.001$. 
Table 5

The Effect of a Computer-based and a Didactic Intervention on Nutrition Knowledge (\# correct) per Category

\begin{tabular}{cccc} 
& \multicolumn{3}{c}{ Experts Advice (10 Questions) } \\
& Baseline & Post & Follow-Up \\
\hline CON & $5.1 \pm 0.15$ & $5.9 \pm 0.20$ & $6.1 \pm 0.17$ \\
CBI & $5.0 \pm 0.15$ & $6.4 \pm 0.20$ & $6.5 \pm 0.17$ \\
TDI & $5.0 \pm 0.16$ & $6.5 \pm 0.21$ & $6.1 \pm 0.18$ \\
& & & \\
CON & $30.3 \pm 0.96$ & $30.1 \pm 0.91^{\mathrm{a}}$ & $30.0 \pm 0.86^{\mathrm{c}}$ \\
CBI & $29.2 \pm 0.97$ & $37.3 \pm 0.93^{* * * \mathrm{~b}}$ & $37.9 \pm 0.88^{* * * \mathrm{~d}}$ \\
TDI & $31.2 \pm 0.98$ & $36.0 \pm 0.94^{* * * \mathrm{~b}}$ & $34.5 \pm 0.88^{\mathrm{e}}$ \\
& & & \\
CON & $4.0 \pm 0.19$ & Food Choices (10 Questions) & $3.9 \pm 0.18$ \\
CBI & $4.0 \pm 0.19$ & $4.4 \pm 0.19$ & $4.5 \pm 0.19$ \\
TDI & $3.9 \pm 0.19$ & $4.6 \pm 0.19$ & $4.4 \pm 0.19$ \\
& & $4.7 \pm 0.19$ & \\
CON & $9.2 \pm 0.51$ & Health $(28$ Questions) & $9.7 \pm 0.47^{\mathrm{i}}$ \\
CBI & $9.1 \pm 0.52$ & $9.6 \pm 0.45^{\mathrm{f}}$ & $14.5 \pm 0.47^{* * * \mathrm{j}}$ \\
TDI & $11.1 \pm 0.53$ & $13.6 \pm 0.46^{* * * \mathrm{~g}}$ & $11.1 \pm 0.48^{\mathrm{k}}$ \\
\hline
\end{tabular}

^Nutrition Knowledge Questionnaire developed by Parmenter \& Wardle (1999). Values represented indicate mean \pm SEM.

${ }^{\mathrm{a}-\mathrm{k}}$ Groups in columns with different letters are statistically different $\mathrm{p}<0.05$.

*** Indicates increase from baseline to post and/or baseline to follow-up, $\mathrm{p}<0.001$.

Hypothesis 2:

The CBI group will have greater success in maintaining/ attaining a healthy body mass index compared to both the TDI and control groups post-intervention and at follow-up.

Data analysis revealed a significant effect of treatment on body mass index ( $p<$ 0.001 ) (Table 6). At baseline $32.2 \%$ of CON, $28.6 \%$ of CBI, and $41.0 \%$ of TDI participants were overweight or obese, having a BMI over 25 (Table 6a). However, there 
was no significant difference in average body mass index among the three groups at baseline. Overweight and obesity is defined by a BMI greater than 25 . The mean body mass index at baseline for all participants was $24.0 \pm 0.3$. There was no change in the CON group from baseline to post, while they did demonstrate an increase in BMI from baseline to follow-up $(\mathrm{p}<0.01)$. The CBI group was the only group that demonstrated a decrease in BMI from pre to post $(\mathrm{p}<0.001)$. The CBI group also demonstrated a further decrease in BMI from post to follow-up $(\mathrm{p}<0.01)$ while the TDI group showed an increase from post to follow-up $(p<0.05)$. From baseline to follow-up, the CBI group demonstrated a decrease in BMI $(\mathrm{p}<0.001)$.

While the variance in BMI among treatment groups appears quite large among the groups, the variance in BMI over time is quite small within groups. The mean difference from baseline to follow-up for the $\mathrm{CON}$ group was $0.33 \pm 0.11$. The mean difference from baseline to follow-up for the CBI group was $-0.55 \pm 0.11$. The mean difference from baseline to follow-up for the TDI group was $0.13 \pm 0.09$.

Table 6

The Effect of a Computer-based and a Didactic Intervention on Average BMI Over Time

\begin{tabular}{llll}
\hline & Baseline & Post & Follow-Up \\
\hline CON & $23.8 \pm 0.54$ & $23.9 \pm 0.54^{\mathrm{a}, \mathrm{b}}$ & $24.1 \pm 0.53^{\mathrm{c}, \mathrm{d}^{*}}$ \\
CBI & $23.2 \pm 0.55$ & $23.0 \pm 0.54^{\mathrm{a}^{* * *}}$ & $22.7 \pm 0.54^{\mathrm{c} * *, * * *}$ \\
TDI & $25.1 \pm 0.55$ & $25.0 \pm 0.55^{\mathrm{b}}$ & $25.2 \pm 0.54^{\mathrm{d}^{*}}$ \\
\hline
\end{tabular}

Values represented indicate mean $\pm \mathrm{SEM}$.

${ }^{a-d}$ Groups in columns with different letters are statistically different $\mathrm{p}<0.05$.

$* * *$ Indicates decrease from baseline to post and/or baseline to follow-up, $\mathrm{p}<0.001$;

** Indicates decrease post to follow-up, $\mathrm{p}<0.01$;

* Indicates increase post to follow-up, $\mathrm{p}<0.05$. 
Table 6a

The effect of the a computer-based and a didactic intervention on the Change in BMI Categories Over Time

\begin{tabular}{|c|c|c|c|c|c|c|}
\hline & & $\begin{array}{c}\text { BMI }< \\
18.5\end{array}$ & $\begin{array}{c}\text { BMI 18.5- } \\
24.9\end{array}$ & $\begin{array}{c}\text { BMI 25- } \\
29.9\end{array}$ & BMI 30-39.9 & $\mathrm{BMI}>40$ \\
\hline \multirow[t]{4}{*}{$\mathrm{CON}$} & $\mathrm{n}=87$ & & & & & \\
\hline & Baseline & $7(8.0 \%)$ & $52(59.8 \%)$ & $20(23.0 \%)$ & $8(9.2 \%)$ & 0 \\
\hline & Post & $5(5.7 \%)$ & $52(59.8 \%)$ & $20(23.0 \%)$ & $10(11.5 \%)$ & 0 \\
\hline & Follow-Up & $6(6.9 \%)$ & $50(57.5 \%)$ & $21(24.1 \%)$ & $10(11.5 \%)$ & 0 \\
\hline \multirow[t]{4}{*}{$\mathrm{CBI}$} & $\mathrm{n}=84$ & & & & & \\
\hline & Baseline & $7(8.3 \%)$ & $53(63.1 \%)$ & $18(21.4 \%)$ & $5(6.0 \%)$ & $1(1.2 \%)$ \\
\hline & Post & $8(9.5 \%)$ & $51(60.7 \%)$ & $19(22.6 \%)$ & $5(6.0 \%)$ & $1(1.2 \%)$ \\
\hline & Follow-Up & $8(9.5 \%)$ & $56(66.7 \%)$ & $13(15.5 \%)$ & $7(8.3 \%)$ & 0 \\
\hline \multirow[t]{4}{*}{ TDI } & $\mathrm{n}=83$ & & & & & \\
\hline & Baseline & $4(4.8 \%)$ & $45(54.2 \%)$ & $18(21.7 \%)$ & $14(16.9 \%)$ & $2(2.4 \%)$ \\
\hline & Post & $5(6.0 \%)$ & $44(53.0 \%)$ & $18(21.7 \%)$ & $15(18.1 \%)$ & $1(1.2 \%)$ \\
\hline & Follow-Up & $5(6.0 \%)$ & $44(53.0 \%)$ & $17(20.5 \%)$ & $16(19.3 \%)$ & $1(1.2 \%)$ \\
\hline
\end{tabular}

Hypothesis 3:

The CBI group will show a decrease in fat intake, especially saturated fat, an increase in fruit, vegetable, and fiber intake, and increased consumption of low-fat dairy products compared to both the TDI and control groups post-intervention and at follow-up.

Kcal

Average daily kilocalorie intake was assessed using two non-consecutive 24-hour recalls (Table 7). There was no significant time by treatment interaction. The 24 -hour recalls revealed an average kilocalorie intake of approximately $1850 \mathrm{kcal}$ across the three groups at baseline, with no significant differences among groups. None of the groups showed a change in energy intake across the 3 time periods. 
Table 7

The Effect of a Computer-based and a Didactic Intervention on Average Kilocalories

Consumed per Day Based on Two Non-consecutive 24-hour Recalls

\begin{tabular}{cccc}
\hline & Baseline & Post & Follow-Up \\
\hline CON & $1971.8 \pm 77.4$ & $1910.3 \pm 71.7$ & $1948.0 \pm 51.5$ \\
CBI & $1651.4 \pm 78.8$ & $1588.2 \pm 73.0$ & $1625.8 \pm 52.4$ \\
TDI & $1914.4 \pm 79.2$ & $1749.3 \pm 73.4$ & $1875.8 \pm 52.7$ \\
\hline
\end{tabular}

Values represented indicate mean \pm SEM.

Fat (Table 8 and 9)

Total dietary fat intake was assessed using two methods, two non-consecutive 24-

hour recalls and a Food Frequency Questionnaire. The 24 -hour recalls revealed an average fat intake of approximately $69 \mathrm{~g}$ per day across the three groups at baseline.

However, there were no significant main effects of group or time or group by time interaction. In contrast, the FFQ did reveal a significant time by treatment interaction $(p<0.001)$ in regards to fat intake (Table 9). Although the 24-hour recall data showed a trend toward lower fat intake in the CBI group, baseline data from the FFQ suggests that the CBI group was consuming more fat per day than the TDI group $(\mathrm{p}<0.05)$. The FFQ also indicated a decrease in fat intake by the CBI group from baseline to post $(p<0.001)$ as well as a decrease from baseline to follow-up $(\mathrm{p}<0.001)$. At post and follow-up data collection there was no difference among the intervention groups, but the CON group reported consuming a greater amount of fat than the CBI group $(\mathrm{p}<0.05)$.

The discrepancies among the 24-hour recalls and the FFQ may possibly be due to the CBI's group misunderstanding regarding completion of the FFQ. As a result, the fat intake may have been overestimated at baseline, which would have made the reduction if fat intake look more drastic than it actually was. 
Table 8

The Effect of a Computer-based and a Didactic Intervention on Average Fat (g) Intake per Day as Measured by Two Non-consecutive 24-hour Recalls

\begin{tabular}{cccc} 
& \multicolumn{3}{c}{ Fat $(\mathrm{g})$} \\
CON & Baseline & Post & Follow-Up \\
CBI & $71.8 \pm 3.51$ & $72.0 \pm 3.20$ & $71.6 \pm 2.59$ \\
TDI & $62.6 \pm 3.57$ & $57.7 \pm 3.25$ & $58.8 \pm 2.64$ \\
& $71.1 \pm 3.60$ & $64.8 \pm 3.27$ & $72.3 \pm 2.65$ \\
CON & $646.4 \pm 31.5$ & Average intake of calories from fat \\
CBI & $563.2 \pm 32.1$ & $647.5 \pm 28.8$ & $644.1 \pm 23.3$ \\
TDI & $640.1 \pm 32.3$ & $519.6 \pm 29.3$ & $528.7 \pm 23.7$ \\
\hline
\end{tabular}

Values represented indicate mean \pm SEM.

Table 9

The Effect of a Computer-based and a Didactic Intervention on Average Fat (g) Intake per

Day as Measured by Food Frequency Questionnaire (FFQ)

\begin{tabular}{cccl}
\hline & Baseline & Post & Follow-Up \\
\hline CON & $61.1 \pm 6.89^{\mathrm{a}, \mathrm{b}}$ & $51.8 \pm 4.16$ & $56.7 \pm 4.19^{\mathrm{c}}$ \\
CBI & $83.8 \pm 7.01^{\mathrm{a}}$ & $50.3 \pm 4.24^{* * * * *}$, & $42.0 \pm$ \\
& & & $4.26^{\mathrm{d}^{* * *}}$ \\
TDI & $55.5 \pm 7.05^{\mathrm{b}}$ & $51.3 \pm 4.26$ & $53.8 \pm$ \\
\hline
\end{tabular}

Values represented indicate mean $\pm \mathrm{SEM}$.

${ }^{\mathrm{a}-\mathrm{d}}$ Groups in columns with different letters are statistically different $\mathrm{p}<0.05$.

*** Indicates decrease from baseline to post and/or baseline to follow-up $\mathrm{p}<0.001$;

* Indicates decrease from post to follow-up, $\mathrm{p}<0.05$.

Saturated Fat

The 24-hour recall was used to assess saturated fat intake (Table 10). The $24-$

hour recalls revealed an average saturated fat intake of approximately $24 \mathrm{~g}$ per day across

the three groups at baseline. On average, the CON, CBI, and TEI groups consumed $35.4 \%, 34.6 \%$, and $34.4 \%$, respectively, of their fat in saturated form. There were no significant differences among the groups, across the time periods, or group by time 
period interaction. No differences were noted in the groups for the daily percent of total fat consumed as saturated for any of the data collection periods.

Table 10

The Effect of a Computer-based and a Didactic Intervention on Average Saturated Fat Intake per Day as Measured by Two Non-consecutive 24-hour Recalls

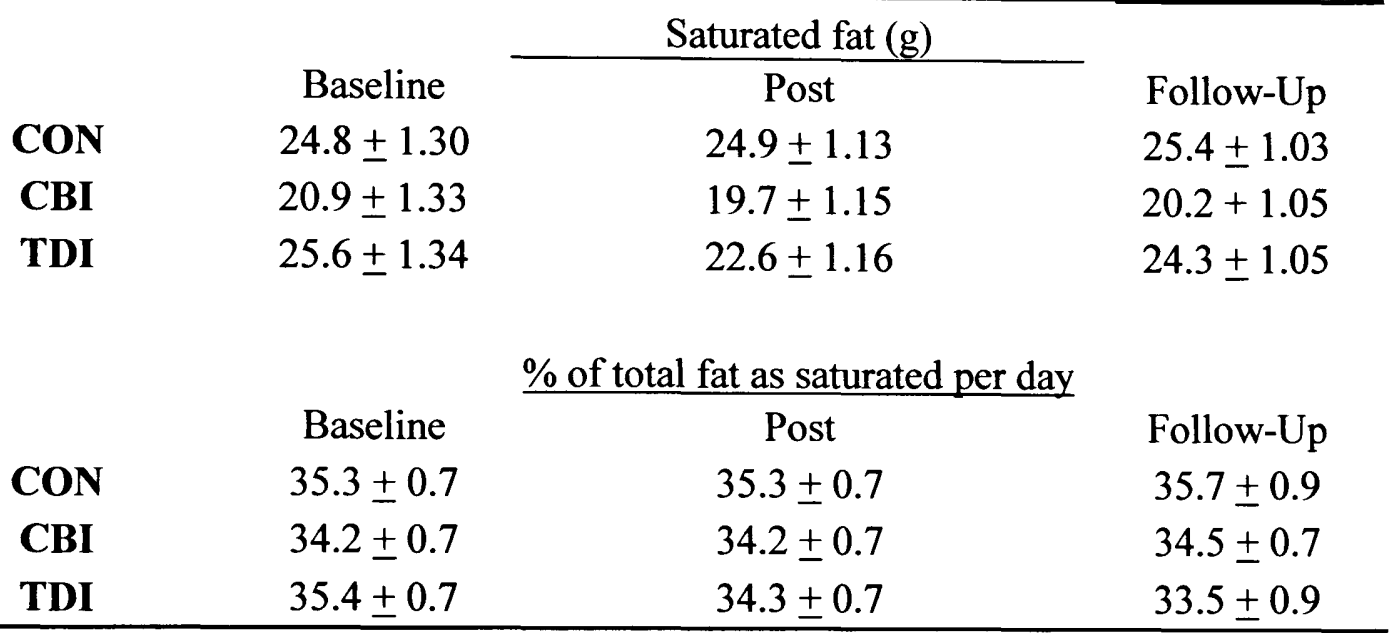

Values represented indicate mean \pm SEM.

Fiber

Fiber was assessed using two methods, two non-consecutive 24-hour recalls and a Food Frequency Questionnaire. The 24 -hour recalls revealed an average fiber intake of approximately $10.7 \mathrm{~g}$ per day across the three groups at baseline (Table 11). There were no significant main effects of group or time period and no significant group by time interaction for fiber intake.

Table 11

The Effect of a Computer-based and a Didactic Intervention on Average Fiber (g) Intake per Day as Measured by Two Non-consecutive 24-hour Recalls

\begin{tabular}{crrr} 
& \multicolumn{1}{c}{ Baseline } & \multicolumn{1}{c}{ Post } & Follow-Up \\
\hline CON & $10.8 \pm 0.56$ & $10.8 \pm 0.59$ & $12.4 \pm 0.52$ \\
CBI & $9.7 \pm 0.57$ & $9.6 \pm 0.60$ & $10.6 \pm 0.53$ \\
TDI & $11.6 \pm 0.58$ & $10.6 \pm 0.61$ & $11.0 \pm 0.54$
\end{tabular}

Values represented indicate mean $\pm \mathrm{SEM}$. 
On the other hand, the FFQ revealed a significant time by treatment interaction $(p<0.025)$. There was a difference in fiber intake at baseline among the groups with the CBI group having a higher intake than the TDI group $(p<0.05)$ (Table 12). This outcome is inconsistent with typical dietary patterns. Individuals with high fat intakes in general do not also have high fiber intake and therefore again causes concern regarding the validity of the FFQ in the CBI group. The FFQ also indicated a decrease in fiber intake by the CBI group from baseline to post $(\mathrm{p}<0.001)$ as well as a decrease from baseline to follow-up ( $<<0.001)$. At post and follow-up data collection there were no differences among the groups.

Table 12

The Effect of a Computer-based and a Didactic Intervention on Average Fiber (g) Intake per Day as Measured by Food Frequency Questionnaire (FFQ)

\begin{tabular}{clll}
\hline & Baseline & Post & Follow-Up \\
\hline CON & $9.4 \pm 0.96^{\mathrm{ab}}$ & $7.9 \pm 0.66$ & $7.9 \pm 0.54$ \\
CBI & $12.3 \pm 0.97^{\mathrm{b}}$ & $8.4 \pm 0.67^{* * *}$ & $7.5 \pm 0.55 * * *$ \\
TDI & $8.9 \pm 0.98^{\mathrm{a}}$ & $7.9 \pm 0.68$ & $7.9 \pm 0.56$ \\
\hline
\end{tabular}

Values represented indicate mean \pm SEM.

${ }^{\mathrm{a}, \mathrm{b}}$ Groups in columns with different letters are statistically different, $\mathrm{p}<0.05$.

*** Indicates decrease from baseline to post and/or baseline to follow-up, $\mathrm{p}<0.001$.

Fruits and Vegetable Intake

Fruit and vegetable intake was assessed using two methods, two non-consecutive 24-hour recalls and a Food Frequency Questionnaire. The 24 -hour recalls revealed an average fruit and vegetable intake of approximately 1.36 servings per day across the three groups at baseline (Table 13). This low fruit and vegetable intake is consistent with a 
low fiber intake. There were no significant main effects of group or time period and no significant group by time interaction for fruit and vegetable intake.

The FFQ revealed a higher fruit and vegetable intake across the three groups of 3.0 servings per day at baseline, with no difference noted among the groups (Table 14). There was, however, a significant effect of treatment on fruit and vegetable intake $(p<0.036)$. The FFQ indicated a decrease in fruit and vegetable intake by the CBI group from baseline to post $(p<0.001)$ as well as a decrease from baseline to follow-up $(p<0.001)$. Again, these baseline results for the CBI group are not probably to be accurate so comparisons are not valid for the FFQ. There were no differences among the groups at post and follow-up.

Table 13

The Effect of a Computer-based and a Didactic Intervention on Fruit and Vegetable Intake(servings/day) as Reported by Two Non-consecutive 24-hour Recalls

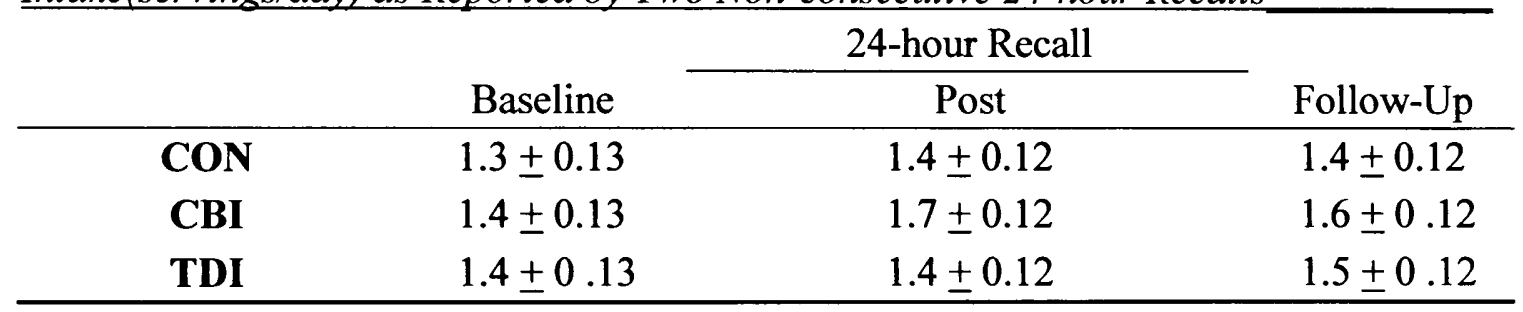

Values represented indicate mean $\pm \mathrm{SEM}$.

Table 14

The Effect of a Computer-based and a Didactic Intervention on Fruit and Vegetable Intake (servings/day) as Reported by Food Frequency Questionnaire (FFQ)

Food Frequency Questionnaire

\begin{tabular}{ccll} 
& Baseline & \multicolumn{1}{c}{ Post } & Follow-Up \\
\hline CON & $2.5 \pm 0.28$ & $2.2 \pm 0.20$ & $2.0 \pm 0.17$ \\
CBI & $3.6 \pm 0.29$ & $2.3 \pm 0.21^{* * *}$ & $2.4 \pm 0.17^{* * *}$ \\
TDI & $2.8 \pm 0.29$ & $2.4 \pm 0.21$ & $2.3 \pm 0.17$ \\
\hline
\end{tabular}

Values represented indicate mean \pm SEM.

*** Indicates increase from baseline to post and/or baseline to follow-up, $\mathrm{p}<0.001$. 
Dairy

Dairy intake was assessed using two non-consecutive 24-hour recalls (Table 15ac). When dairy products were consumed, participants were asked to specify whether a reduced fat $(2 \%, 1 \%$, fat-free) or regular (whole) dairy product was consumed. Total dairy intake (all fat levels) across all three groups was approximately one serving per day at baseline. There was a significant time by treatment interaction $(p<0.001)$ for total dairy intake. At baseline the TDI group consumed more total dairy than the CBI group $(p<0.001)$. The CBI group increased their total dairy intake from baseline to post $(p<0.01)$, but decreased from post to follow-up $(p<0.05)$. The TDI group decreased their total dairy intake from baseline to post $(\mathrm{p}<0.001)$ as well as baseline to follow-up $(p<0.001)$. There was also a difference among the groups at follow-up with the CON group consuming more than both the CBI $(\mathrm{p}<0.01)$ and TDI $(\mathrm{p}<0.01)$ groups.

According to the 24 -hour recalls the average intake of regular (whole) dairy at baseline was approximately half of a serving per day for all three groups (Table 15a). Participants in general consumed low-fat milk at school (as served on standard lunch tray) and whole fat versions at home. There was a significant time by group interaction of treatment on intake of regular dairy $(\mathrm{p}<0.001)$. At baseline the TDI group consumed more regular dairy compared to the $\operatorname{CON}(p<0.001)$ and the CBI group $(p<0.05)$. The CBI group increased the number of servings of their regular dairy intake from baseline to post $(\mathrm{p}<0.029)$. However, the TDI group decreased their regular dairy intake from baseline to post $(\mathrm{p}=0.023)$, post to follow-up $(\mathrm{p}<0.01)$, and baseline to follow-up $(p<0.001)$. Nevertheless, there was no difference among the groups at post or follow-up. 
Low-fat dairy intake (servings/day) averaged approximately one-third of a serving with no difference noted among the groups at baseline (Table 15b). There was a significant group by time period effect on intake of low-fat diary $(p<0.05)$, but the CON group demonstrated an increase in their low-fat dairy intake over the course of the study. The CON group increased their intake from baseline to post and had greater average intake than the TDI group $(p<0.01)$ or the CBI group ( $<<0.001)$ at follow-up. The 24hour recall also revealed an increase by the $\mathrm{CON}$ group from baseline to follow-up $(\mathrm{p}=0.007)$. At follow-up the CON group showed a higher intake of low-fat dairy compared to both the CBI $(p<0.001)$ and TDI $(p=0.002)$ groups. This is likely related to the higher free lunch eligibility of the participants in the CON group compared to the other groups. Although there is knowledge that there was a change in eligibility requirements, actual participants eligible for free or reduced lunch was not measured during the course of the study and changes therefore can only be speculated.

Macronutrient Intake

The macronutrients protein and carbohydrate as well as cholesterol intakes were analyzed using the 24-hour recall. There were no significant main effects of group or time period or interactions of group by time period noted in intake of protein (Table 16), carbohydrate (Table 17), or cholesterol (Table 18). 
The Effect of a Computer-based and a Didactic Intervention on Regular (whole)

Dairy Intake (servings/day) as Reported by Two Non-consecutive 24-hour Recalls

\begin{tabular}{cccl}
\hline & Baseline & Post & Follow-Up \\
\hline CON & $0.4 \pm 0.08^{\mathrm{a}, \mathrm{b}}$ & $0.4 \pm 0.07$ & $0.4 \pm 0.05$ \\
CBI & $0.3 \pm 0.08^{\mathrm{b}}$ & $0.5 \pm 0.07^{*}$ & $0.4 \pm 0.06$ \\
TDI & $0.8 \pm 0.08^{\mathrm{a}}$ & $0.5 \pm 0.07^{*}$ & $0.3 \pm 0.05^{* *},{ }^{* * *}$ \\
\hline
\end{tabular}

Values represented indicate mean \pm SEM.

${ }^{\mathrm{a}, \mathrm{b}}$ Groups in columns with different letters are statistically different, $\mathrm{p}<0.05$.

* Indicates change from baseline to post, $\mathrm{p}<0.05$;

** Indicates decrease from post to follow-up, $\mathrm{p}<0.01$;

$* * *$ Indicates decrease from baseline to follow-up, $\mathrm{p}<0.001$.

Table $15 b$

The Effect of a Computer-based and a Didactic Intervention on Low-fat (2\%, $1 \%$, fatfree) Dairy Intake (servings/day) as Reported by Two Non-consecutive 24-hour Recalls

\begin{tabular}{cccc}
\hline & Baseline & Post & Follow-Up \\
\hline CON & $0.5 \pm 0.06$ & $0.6 \pm 0.06^{\mathrm{a}}$ & $0.7 \pm 0.07^{\mathrm{c**}}$ \\
CBI & $0.3 \pm 0.06$ & $0.4 \pm 0.07^{\mathrm{ab}}$ & $0.3 \pm 0.07^{\mathrm{d}}$ \\
TDI & $0.4 \pm 0.06$ & $0.3 \pm 0.07^{\mathrm{b}}$ & $0.4 \pm 0.07^{\mathrm{d}}$ \\
\hline
\end{tabular}

Values represented indicate mean \pm SEM.

${ }^{\mathrm{a}-\mathrm{d}}$ Groups in columns with different letters are statistically different, $\mathrm{p}<0.01$.

** Indicates an increase from baseline to follow-up, $\mathrm{p}<0.01$.

Table 15c

The Effect of a Computer-based and a Didactic Intervention on Total Dairy Intake (servings/day) as Reported by Two Non-consecutive 24-hour Recalls

\begin{tabular}{cccc} 
& Baseline & Post & Follow-Up \\
\hline CON & $0.9 \pm 0.10^{\mathrm{a}, \mathrm{b}}$ & $1.0 \pm 0.09$ & $1.0 \pm 0.09^{\mathrm{c}}$ \\
CBI & $0.6 \pm 0.10^{\mathrm{a}}$ & $0.9 \pm 0.10^{* *}$ & $0.7 \pm 0.09^{\mathrm{d}^{*}}$ \\
TDI & $1.1 \pm 0.10^{\mathrm{b}}$ & $0.9 \pm 0.10^{*}$ & $0.7 \pm 0.09^{\mathrm{d}^{* * *}}$ \\
\hline
\end{tabular}

Values represented indicate mean $\pm \mathrm{SEM}$.

${ }^{a-d}$ Groups in columns with different letters are statistically different, $p<0.05$.

* Indicates a decrease from post to follow-up, and a decrease from baseline to post, $\mathrm{p}<0.05$;

** Indicates an increase from baseline to post, $\mathrm{p}<0.01$;

*** Indicates decrease from baseline to follow-up, $\mathrm{p}<0.001$. 
Table 16

The Effect of a Computer-based and a Didactic Intervention on Average Protein (g) Intake per Day as Measured by Two Non-consecutive 24-hour Recalls

\begin{tabular}{cccc} 
& Baseline & Post & Follow-Up \\
\hline CON & $66.3 \pm 3.55$ & $65.9 \pm 3.43$ & $68.0 \pm 3.30$ \\
CBI & $59.3 \pm 3.61$ & $58.8 \pm 3.49$ & $59.2 \pm 3.36$ \\
TDI & $73.0 \pm 3.63$ & $64.1 \pm 3.51$ & $72.2 \pm 3.38$ \\
\hline
\end{tabular}

Values represented indicate mean \pm SEM.

Table 17

The Effect of a Computer-based and a Didactic Intervention on Average Carbohydrates (g) Intake per Day as Measured by Two Non-consecutive 24-hour Recalls

\begin{tabular}{llll} 
& Baseline & Post & Follow-Up \\
\hline CON & $256.3 \pm 10.22$ & $251.9 \pm 9.86$ & $257.8 \pm 8.0$ \\
CBI & $218.1 \pm 10.40$ & $212.5 \pm 10.04$ & $236.6 \pm 8.14$ \\
TDI & $250.3 \pm 10.46$ & $225.4 \pm 10.10$ & $239.6 \pm 8.19$ \\
\hline
\end{tabular}

Values represented indicate mean \pm SEM.

Table 18

The Effect of a Computer-based and a Didactic Intervention on Average Cholesterol (mg) Intake per Day as Measured by Two Non-consecutive 24-hour Recalls

\begin{tabular}{cccc} 
& Baseline & Post & Follow-Up \\
\hline CON & $190.5 \pm 12.90$ & $194.4 \pm 11.57$ & $207.7 \pm 13.11$ \\
CBI & $172.4 \pm 13.13$ & $172.2 \pm 11.78$ & $182.6 \pm 13.34$ \\
TDI & $202.6 \pm 13.21$ & $177.2 \pm 11.85$ & $201.5 \pm 13.42$ \\
\hline
\end{tabular}

Values represented indicate mean \pm SEM.

a La Carte Lunches

Based on the responses of the 24-hour recalls, purchases of a La carte foods were assessed (Table 19). At baseline the groups demonstrated similar patterns for lunch purchases with no differences noted among the groups for selection of pizza, vending machine foods, or standard school lunch tray. Groups averaged about one pizza and one 
vending machine purchase per day. There were no differences among the groups, for the number of times the students purchased food from the a la carte pizza line among the data collection points. There was, however, a significant effect of treatment on purchase of vending machine snacks $(p<0.001)$ Purchasing of vending machine snacks increased from baseline to post $(\mathrm{p}=0.002)$ and from baseline to follow-up $(\mathrm{p}<0.001)$ for the TDI group and for the CON group from baseline to follow-up $(\mathrm{p}=0.04)$. The CBI group, however, remained constant in their vending machine purchases throughout the study.

Table 19

The Effect of a Computer-based and a Didactic Intervention on Daily a La Carte Lunch Purchases as Reported by Two Non-consecutive 24-hour Recalls

\begin{tabular}{cccc}
\hline & Baseline & $\underline{\text { Pizza }}$ & \\
& $0.9 \pm 0.02$ & $0.9 \pm 0.02$ & $0.8 \pm 0.02$ \\
CON & $0.9 \pm 0.02$ & $0.9 \pm 0.02$ & $0.8 \pm 0.02$ \\
CBI & $0.9 \pm 0.02$ & $0.9 \pm 0.02$ & $0.9 \pm 0.02$ \\
TDI & & Vonding Machine & \\
& & Post & Follow-Up \\
\hline & $0.8 \pm 0.02$ & $0.9 \pm 0.02$ & $0.9 \pm 0.02^{*}$ \\
CON & $0.9 \pm 0.02$ & $0.9 \pm 0.02$ & $0.8 \pm 0.02$ \\
CBI & $0.8 \pm 0.02$ & $0.9 \pm 0.02^{* *}$ & $0.9 \pm 0.02^{* * *}$ \\
\hline TDI & &
\end{tabular}

Values represented indicate mean \pm SEM. Analysis used square root transformation.

*Indicates increase from baseline to follow-up, $\mathrm{p}<0.05$;

$* *$ Indicates increase from baseline to post, $\mathrm{p}<0.01$;

$* * *$ Indicates increase from baseline to follow-up, $\mathrm{p}<0.001$.

Soda Consumption

The 24-hour recalls were used to assess daily soda intake (Table 20). There was a significant interaction of group by time period $(\mathrm{p}<0.01)$, in addition to a significant effect of treatment on soda intake $(\mathrm{p}<0.034)$. At baseline, the 24 -hour recall revealed that there 
was no difference in soda consumption among the groups with participants averaging approximately 19.5 ounces of soda per day. There were no changes noted for the CON group over any of the data collection periods. From baseline to post, there was a decrease in soda consumption in both the CBI $(\mathrm{p}=0.003)$ and the TDI $(\mathrm{p}=0.005)$. However, the TDI group increased their soda consumption from post to follow-up $(\mathrm{p}=0.034)$.

Table 20

The Effect of a Computer-based and a Didactic Intervention on Average Amount of Soda (oz) Consumed per Day as Reported by Two Non-consecutive 24-hour Recalls

\begin{tabular}{ccll}
\hline & Baseline & Post & Follow-Up \\
\hline CON & $17.7 \pm 1.55$ & $18.1 \pm 1.37$ & $16.8 \pm 1.33$ \\
CBI & $20.3 \pm 1.60$ & $15.2 \pm 1.41^{* *}$ & $17.6 \pm 1.37$ \\
TDI & $20.3 \pm 1.59$ & $15.4 \pm 1.41^{* *}$ & $19.1 \pm 1.36^{*}$ \\
\hline
\end{tabular}

Values represented indicate mean \pm SEM.

*Indicates increase from post to follow-up, $p<0.05$;

**Indicates decrease from baseline to post, $\mathrm{p}<0.01$.

Meals Skipped

Based on the responses of the 24-hour recalls, the number of times participants skipped meals was assessed. (Table 21) On average, participants skipped one meal per day. There was an group by time interaction in the frequency of meals skipped. The number of meals skipped remained constant throughout the study for the CON and TDI groups. At baseline, the CBI group reported skipping more meals than the TDI $(p=0.006)$ and $\mathrm{CON}$ group $(\mathrm{p}=0.003)$. Only the $\mathrm{CBI}$ group significantly reduced the number of meals skipped from both baseline to post and baseline to follow-up $(p<0.001)$. At baseline, the CBI group reported that $61.1 \%$ skipped breakfast, $21.7 \%$ skipped lunch, and $4.1 \%$ skipped dinner. However, at post evaluation, these numbers were reduced to $41.3 \%, 15.7 \%, 4.7 \%$, respectively. There were no differences among the TDI and CON 
groups at any point in the intervention and there were no differences among the three groups at post or follow-up.

Table 21

The Effect of a Computer-based and a Didactic Intervention on Average Number of Meals Skipped as Reported by Two Non-consecutive 24-hour Recalls

\begin{tabular}{llll}
\hline & Baseline & \multicolumn{1}{c}{ Post } & Follow-Up \\
\hline CON & $1.3 \pm 0.04^{\mathrm{a}}$ & $1.2 \pm 0.05$ & $1.2 \pm 0.04$ \\
CBI & $1.5 \pm 0.05^{\mathrm{b}}$ & $1.2 \pm 0.05^{* * *}$ & $1.2 \pm 0.04^{* * *}$ \\
TDI & $1.3 \pm 0.05^{\mathrm{a}}$ & $1.3 \pm 0.05$ & $1.3 \pm 0.04$ \\
\hline
\end{tabular}

Analysis used square root transformation. Values represented indicate mean \pm SEM.

${ }^{\mathrm{a}, \mathrm{b}}$ Groups in columns with different letters are statistically different, $\mathrm{p}<0.01$.

${ }^{* * *}$ Indicates decrease from baseline to post and/or baseline to follow-up, $\mathrm{p}<0.001$.

Hypothesis 4:

The CBI group will have an increase in physical activity level compared to both the TDI and control groups post-intervention and at follow-up.

Total physical activity was calculated for each participant using the PAQ-a (Table 22). A range of scores was assigned to each activity level. A score of 8-15.9 was classified as sedentary, 16-23.9 lightly active, 24-31.9 moderately active, and a score above 32 suggested vigorous activity.

Table 22

The Effect of a Computer-based and a Didactic Intervention on Self-Reported Physical Activity as Reported on $P A \hat{Q}$

\begin{tabular}{ccll}
\hline & Baseline & \multicolumn{1}{c}{ Post } & Follow-Up \\
\hline CON & $17.7 \pm 0.66$ & $18.6 \pm 0.66$ & $18.8 \pm 0.62^{\mathrm{a}}$ \\
CBI & $17.6 \pm 0.68$ & $19.6 \pm 0.67^{* *}$ & $21.0 \pm 0.63^{* * * \mathrm{~b}}$ \\
TDI & $17.5 \pm 0.68$ & $18.3 \pm 0.68$ & $18.5 \pm 0.64^{\mathrm{a}}$ \\
\hline
\end{tabular}

^PAQ-a developed by Crocker et al (1997). Values represented indicate mean $\pm \mathrm{SEM}$.

${ }^{\mathrm{a}, \mathrm{b}} \mathrm{Groups}$ in columns with different letters are statistically different, $\mathrm{p}<0.05$.

$* *$ Indicates increase from baseline to post, $\mathrm{p}<0.01$;

$* * *$ Indicates increase from baseline to follow-up, $\mathrm{p}<0.001$. 
At baseline, there was no difference in self-reported activity totals among the three groups with the overall average score of 17.5 or lightly active. There was a significant interaction effect of group by time period on physical activity $(p<0.05)$. Post activity scores indicated that the CBI group had a significant increase in physical activity $(p=0.005)$ from baseline while the TDI and CON group showed no change in physical activity. The increase in physical activity for the CBI was sustained over time, with the change also noted from baseline to follow-up. At follow-up the CBI group had higher physical activity scores compared to both the CON $(p=0.05)$ and the TDI $(p=0.021)$ group.

These physical activity scores indicated that the majority of the students were sedentary or only lightly active. In fact, during the three data collection periods only a small percentage $(18.4 \%, 16.1 \%$, and $12.6 \%$ respectively), of the CON group reported being moderately to vigorously active on a daily basis. In the CBI group, at baseline $14.3 \%$ reported being moderately to vigorously active on a daily basis, while the number increased to $22.6 \%$ at post, and $28.6 \%$ at follow-up. The TDI group reported $15.7 \%$ participating in moderate to vigorous daily activity at baseline, $22.8 \%$ at post, and $19.3 \%$ at follow-up (Table 22a).

Table 22a

The Effect of a Computer-based and a Didactic Intervention on Percentage of Students Reporting Daily Moderate to Vigorous Physical Activity

\begin{tabular}{cccc}
\hline & Baseline & Post & Follow-Up \\
\hline CON & $18.4 \%$ & $16.1 \%$ & $12.6 \%$ \\
CBI & $14.3 \%$ & $22.6 \%$ & $28.6 \%$ \\
TDI & $15.7 \%$ & $22.8 \%$ & $19.3 \%$ \\
\hline
\end{tabular}

^PAQ-a developed by Crocker at al (1997). 
Hypothesis 5:

The CBI group will increase their self-efficacy of dietary and physical activity habits compared to both the TDI and control groups post-intervention and at follow-up.

There were no significant effects of group, time period or group by time period on perceived self-efficacy, self-confidence for dietary or exercise outcome measures (Table 23).

Hypothesis 6:

The CBI group will increase their perceived social support compared to both the TDI and control groups post-intervention and at follow-up.

Friends

There were no significant effects of group, time period or group by time period on perceived social support, for dietary or exercise by friends (Table 24).

Family (Table 25)

At baseline there was no difference in perceived social support from family among the three groups for any of the subsections. There were not significant differences among the groups, across the time period, nor was there a significant group by time interaction.

Hypothesis 7:

The CBI group will increase their frequency of label reading compared to both the TDI and control groups post-intervention and at follow-up.

The final question on the Food Frequency Questionnaire was used to determine the participants' frequency of label reading (Table 26). There were no differences in how often subjects reported reading among the groups at baseline, post, or follow-up. In general, subjects reported reading labels sometimes $($ Likert $=3)$. 
Table 23

The Effect of a Computer-based and a Didactic Intervention on Perceived SelfEfficacy Scores as Assessed Using Dietary and Exercise Confidence Survey^

\begin{tabular}{|c|c|c|c|}
\hline & \multicolumn{3}{|c|}{ Healthier Eating Habits } \\
\hline & Baseline & Post & Follow-Up \\
\hline CON & $3.0 \pm 0.11$ & $3.1 \pm 0.10$ & $3.2 \pm 0.10$ \\
\hline CBI & $3.0 \pm 0.12$ & $3.1 \pm 0.11$ & $3.2 \pm 0.11$ \\
\hline TDI & $3.2 \pm 0.11$ & $3.3 \pm 0.10$ & $3.1 \pm 0.10$ \\
\hline & \multicolumn{3}{|c|}{ Portion Control } \\
\hline CON & $3.3 \pm 0.10$ & $3.3 \pm 0.10$ & $3.4 \pm 0.10$ \\
\hline CBI & $3.4 \pm 0.12$ & $3.5 \pm 0.11$ & $3.5 \pm 0.11$ \\
\hline TDI & $3.4 \pm 0.11$ & $3.6 \pm 0.10$ & $3.5 \pm 0.10$ \\
\hline & \multicolumn{3}{|c|}{ Salt Intake } \\
\hline CON & $3.3 \pm 0.11$ & $3.4 \pm 0.11$ & $3.4 \pm 0.10$ \\
\hline CBI & $3.0 \pm 0.12$ & $3.3 \pm 0.12$ & $3.2 \pm 0.12$ \\
\hline TDI & $3.2 \pm 0.11$ & $3.2 \pm 0.11$ & $3.3 \pm 0.11$ \\
\hline & \multicolumn{3}{|c|}{ Make Healthier Substitutions } \\
\hline CON & $3.2 \pm 0.11$ & $3.2 \pm 0.09$ & $3.2 \pm 0.10$ \\
\hline CBI & $3.1 \pm 0.12$ & $3.4 \pm 0.10$ & $3.0 \pm 0.12$ \\
\hline TDI & $3.3 \pm 0.11$ & $3.3 \pm 0.09$ & $3.2 \pm 0.10$ \\
\hline & \multicolumn{3}{|c|}{ Make Exercise a Priority } \\
\hline CON & $3.3 \pm 0.10$ & $3.3 \pm 0.09$ & $3.3 \pm 0.10$ \\
\hline CBI & $3.1 \pm 0.11$ & $3.2 \pm 0.10$ & $3.0 \pm 0.10$ \\
\hline TDI & $3.1 \pm 0.10$ & $3.1 \pm 0.09$ & $3.2 \pm 0.10$ \\
\hline & \multicolumn{3}{|c|}{ Maintain an Exercise Program } \\
\hline CON & $3.2 \pm 0.10$ & $3.2 \pm 0.10$ & $3.2 \pm 0.10$ \\
\hline CBI & $3.1 \pm 0.12$ & $3.2 \pm 0.11$ & $3.1 \pm 0.11$ \\
\hline TDI & $3.3 \pm 0.10$ & $3.3 \pm 0.10$ & $3.3 \pm 0.10$ \\
\hline
\end{tabular}

${ }^{\wedge}$ Developed by Sallis (1988). Values represented indicate mean \pm SEM.

Based on Likert scale 1= "I Know I Cannot", 5= "I Know I Can" 
The Effect of a Computer-based and a Didactic Intervention on Perceived Friends' Social Support as Assessed Using Social Support Survey^

\begin{tabular}{cccc} 
& Baseline & Post & Follow-Up \\
\hline CON & $8.9 \pm 0.43$ & Encourage Healthy Eating Habits \\
CBI & $8.4 \pm 0.44$ & $10.2 \pm 0.53$ & $9.8 \pm 0.52$ \\
TDI & $8.4 \pm 0.44$ & $10.5 \pm 0.53$ & $10.5 \pm 0.53$ \\
& \multicolumn{4}{c}{$10.5 \pm 0.53$} \\
CON & $22.2 \pm 1.13$ & Encourage Exercise \\
CBI & $21.2 \pm 1.15$ & $23.3 \pm 1.10$ & $22.7 \pm 1.19$ \\
TDI & $20.9 \pm 1.15$ & $23.8 \pm 1.12$ & $24.7 \pm 1.22$ \\
& & $24.7 \pm 1.12$ & $25.0 \pm 1.22$ \\
CON & $9.7 \pm 0.47^{\mathrm{b}}$ & $10.4 \pm 0.51$ & $10.6 \pm 0.50$ \\
CBI & $10.3 \pm 0.48^{\mathrm{a}, \mathrm{b}}$ & $11.3 \pm 0.52$ & $12.7 \pm 0.51$ \\
TDI & $10.7 \pm 0.48^{\mathrm{a}}$ & $11.8 \pm 0.52$ & $12.2 \pm 0.51$ \\
& & & \\
& & Discourage Healthy Eating Habits & \\
CON & $25.9 \pm 0.14$ & $28.7 \pm 0.17$ & $28.0 \pm 0.19$ \\
CBI & $26.5 \pm 0.14$ & $27.8 \pm 0.17$ & $31.7 \pm 0.20$ \\
TDI & $24.3 \pm 0.14$ & $29.4 \pm 0.17$ & \\
\hline
\end{tabular}

${ }^{\wedge}$ Developed by Sallis et al(1987). Values represented indicate mean \pm SEM.

${ }^{a, b}$ Groups in columns with different letters are statistically different $\mathrm{p}<0.05$. 
Table 25

The Effect of a Computer-based and a Didactic Intervention on Perceived Family Social Support as Assessed Using Social Support Survey^

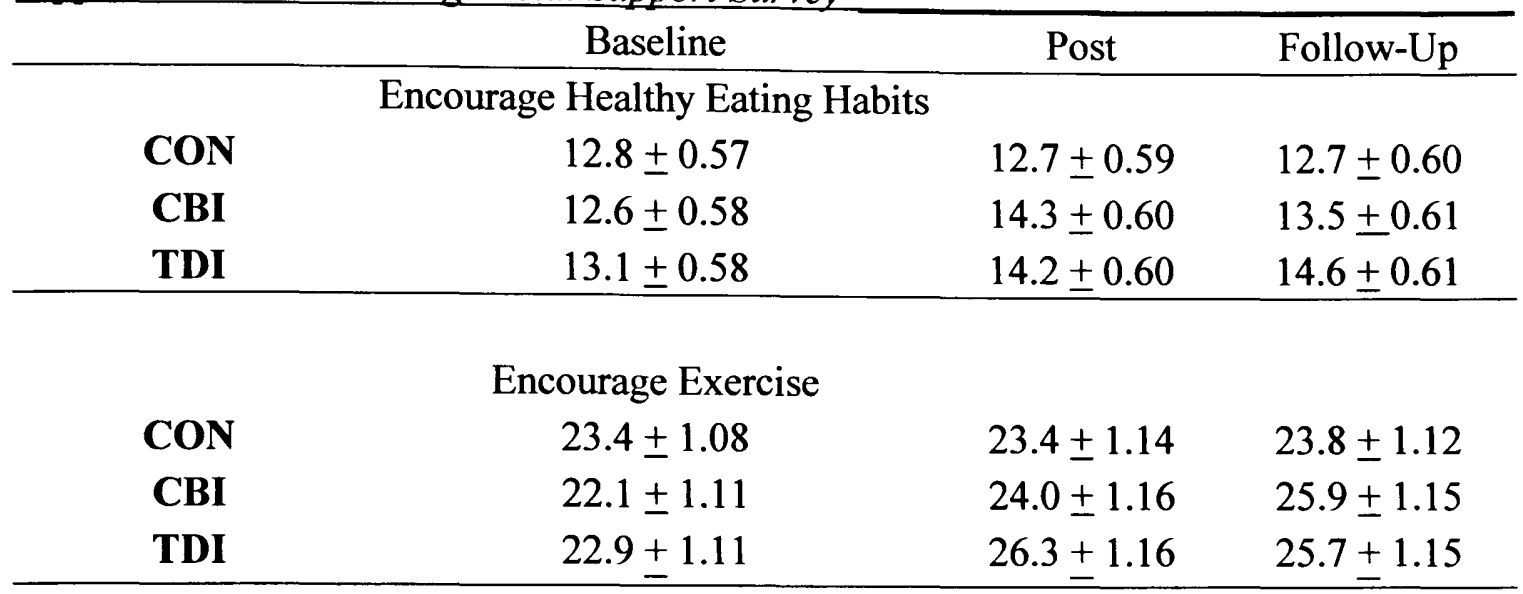

Discourage Healthy Eating Habits

\begin{tabular}{llll} 
CON & $10.8 \pm 0.54$ & $10.9 \pm 0.53$ & $11.7 \pm 0.53$ \\
CBI & $11.9 \pm 0.56$ & $12.2 \pm 0.55$ & $12.6 \pm 0.54$ \\
TDI & $11.4 \pm 0.56$ & $13.4 \pm 0.54$ & $12.7 \pm 0.54$ \\
\hline
\end{tabular}

Discourage Exercise

\begin{tabular}{llll} 
CON & $31.8 \pm 0.20$ & $33.4 \pm 0.20$ & $31.5 \pm 0.21$ \\
CBI & $29.6 \pm 0.21$ & $28.1 \pm 0.21$ & $30.5 \pm 0.21$ \\
TDI & $29.0 \pm 0.21$ & $34.2 \pm 0.21$ & $33.7 \pm 0.21$ \\
\hline
\end{tabular}

${ }^{\wedge}$ Developed by Sallis et al (1987). Values represented indicate mean \pm SEM.

Table 26

The Effect of a Computer-based and a Didactic Intervention on the Frequency of Label Reading

\begin{tabular}{cccc}
\hline & Baseline & Post & Follow-Up \\
\hline CON & $2.85 \pm 0.14$ & $2.83 \pm 0.12$ & $2.92 \pm 0.14$ \\
CBI & $2.7 \pm 0.11$ & $3.04 \pm 0.11^{* *}$ & $3.06 \pm 0.11^{* *}$ \\
TDI & $3.0 \pm 0.11$ & $3.0 \pm 0.11$ & $2.9 \pm 0.11$ \\
\hline
\end{tabular}

Likert Scale: 1 = "Almost Never Read"; 5 = "Almost Always Read".

Values represented indicate mean $\pm \mathrm{SEM}$.

${ }^{* *}$ Indicates increase from baseline to post and/or baseline to follow-up, $\mathrm{p}<0.01$. 


\section{Other Dietary Intake Findings}

Micronutrient Intake (Table 27)

\section{Calcium Intake}

There were no differences among the groups in their calcium intake at any data collection point, nor were there differences within the groups across time.

\section{Iron Intake}

There were no differences among the groups in their calcium intake at any data collection point, nor were there differences within the groups across time.

\section{Folate Intake}

Statistical analysis revealed a significant time by treatment interaction $(p<0.004)$. At baseline the CBI group consumed less folate per day than the TDI group $(p=0.032)$. At post, the CBI group consumed less than the $\mathrm{CON}(\mathrm{p}=0.024)$. At follow-up the $\mathrm{CBI}$ group was also consuming less folate per day than the TDI group $(\mathrm{p}=0.031)$. There were no changes within the group among data collections.

Fast Food Consumption (Table 28)

Based on the responses of the 24-hour recalls, the number of times participants ate fast foods was assessed. At baseline, there were no differences in the number of times the participants consumed food from fast food establishments. On average, one meal out of every three was fast or convenience food. There were no differences among the groups at any of the data collection points, nor were there significant changes for any of the groups over time. 
Table 27

The Effect of a Computer-based and a Didactic Intervention on Micronutrient Intake as Assessed by Two Non-consecutive 24-hour Recalls

\begin{tabular}{cccc} 
& Baseline & Post & \multicolumn{1}{c}{ Follow-Up } \\
\hline & & Calcium $(\mathrm{mg})$ & \\
CON & $611.2 \pm 39.42$ & $655.3 \pm 45.10$ & $641.5 \pm 32.84$ \\
CBI & $488.0 \pm 40.12$ & $539.7 \pm 45.90$ & $579.4 \pm 33.4$ \\
TDI & $721.1 \pm 40.36$ & $693.2 \pm 46.18$ & $626.0 \pm 33.62$ \\
\hline & & Iron $(\mathrm{mg})$ & \\
CON & $11.5 \pm 0.57$ & $11.9 \pm 0.56$ & $11.9 \pm 0.51$ \\
CBI & $10.0 \pm 0.58$ & $9.7 \pm 0.57$ & $11.2 \pm 0.52$ \\
TDI & $12.6 \pm 0.58$ & $11.8 \pm 0.58$ & $11.4 \pm 0.53$ \\
\hline & & & \\
CON & $243.4 \pm 21.75^{\mathrm{a}} \mathrm{b}$ & $265.7 \pm 20.78^{\mathrm{a}}$ & $279.7 \pm 16.62^{\mathrm{a}}$ \\
CBI & $211.1 \pm 22.13^{\mathrm{a}}$ & $186.5 \pm 21.14^{\mathrm{b}}$ & $217.1 \pm 16.91^{\mathrm{a}, \mathrm{b}}$ \\
TDI & $291.9 \pm 22.27^{\mathrm{b}}$ & $241.5 \pm 21.27^{\mathrm{a}, \mathrm{b}}$ & $208.3 \pm 17.02^{\mathrm{b}}$ \\
\hline
\end{tabular}

Values represented indicate mean \pm SEM.

${ }^{a, b}$ Groups in columns with different letters are statistically different, $\mathrm{p}<0.05$.

Table 28

The Effect of a Computer-based and a Didactic Intervention on the Number of Times

Participants Consumed Fast Food as Reported by Two Non-consecutive 24-hour Recalls

\begin{tabular}{cccc}
\hline & Baseline & Post & Follow-Up \\
\hline CON & $0.4 \pm 0.04$ & $0.4 \pm 0.05$ & $0.3 \pm 0.06$ \\
CBI & $0.3 \pm 0.04$ & $0.2 \pm 0.05$ & $0.3 \pm 0.06$ \\
TDI & $0.4 \pm 0.04$ & $0.4 \pm 0.05$ & $0.4 \pm 0.06$ \\
\hline
\end{tabular}

Values represented indicate mean \pm SEM.

Fries and Chips (Table 29)

Based on the responses of the 24-hour recalls, the number of times participants ate french fries or chips was assessed. At baseline the groups demonstrated similar patterns for fries and chips purchases. On average, the participants consumed about $3 / 4$ serving of 
fries or chips per day. There were no differences among the groups at any data collection points in the number of times the students purchased fries or chips.

Table 29

The Effect of a Computer-based and a Didactic Intervention on Servings of French Fries/chips per Day as Reported by Two Non-consecutive 24- hour Recalls

\begin{tabular}{cccc}
\hline & Baseline & Post & Follow-Up \\
\hline CON & $0.8 \pm 0.09$ & $0.8 \pm 0.08$ & $0.7 \pm 0.08$ \\
CBI & $0.8 \pm 0.09$ & $0.6 \pm 0.08$ & $0.6 \pm 0.08$ \\
TDI & $0.6 \pm 0.09$ & $0.7 \pm 0.08$ & $0.7 \pm 0.08$ \\
\hline
\end{tabular}

Values represented indicate mean $\pm \mathrm{SEM}$.

\section{Additional Findings}

\section{$\underline{\text { Session Evaluations }}$}

There were no significant differences among the intervention groups in their satisfaction rating of the session. Each session received favorable ratings by both intervention groups and the average for all the sessions was 4.43 on a 5 -point ( 1 = "would definitely not recommend" to 5 = "would definitely recommend") Likert scale. Of the participants in the CBI group $99 \%$ indicated they would prefer this method rather than receiving printed materials and lectures, however, there was no way to measure preference because each group received only one format. The average Likert score was 5.81 on a 7 -point scale with $1=$ "definitely prefer handouts and lectures" to $7=$ "definitely prefer computer based." 


\section{CHAPTER V}

\section{DISCUSSION AND CONCLUSIONS}

Adolescents, especially minority youth, remain one of the most underserved populations with respect to nutrition and health education. Many of the health educationrelated resources available do not adequately address recommended preventive practices (Skinner et al, 2003). Past intervention studies have resulted in increased knowledge, however, the ultimate goal of health education is to positively influence lifestyle behavior and enhance health through the reduction of risk factors (Hoelsher et al, 2002; Litchfield et al, 2002). A behavioral focus addressing the cognitive, motivational, and skill-building aspects of learning to enhance behavior change in the lifestyle of the target group is essential.

In the practical intervention approach that was used in this study, the term practical means that the adolescent creates actions aimed at a healthy lifestyle. The targeted behaviors included both dietary improvement and incorporation of physical activity. The practical approach aims to increase follow through in everything in life, including self-empowerment to acquire optimal personal health and to accomplish balanced eating to gain both health and pleasure. This also has the potential to improve the adolescents' self-efficacy. Research shows that empowerment, self-efficacy, peer support, and incentives are variables that can affect dietary behaviors (Savoca and Miller, 2001).

\section{Discussion of hypotheses}

In this study an intervention attempting to change lifestyle habits, with an emphasis on diet and physical activity, was delivered via two different methods. 
Previous research with traditional nutrition education focused on increasing knowledge in order to change behavior. This type of intervention has done little to elicit change in food/nutrient intake or increased physical activity patterns (Hoelsher et al, 2002; Sallis et al, 2003; Reynolds et al, 1998). It was hypothesized that the CBI group would demonstrate more positive changes for all parameters in this study because the computerbased version was delivered by a means that adolescents would likely prefer to traditional didactic teaching by telling. In fact we found that the CBI group did make many more positive lifestyle changes than the TDI group and many of the changes were maintained at follow-up. The results of this study indicate that a computer-based method of teaching may have greater potential than traditional methods to improve the lifestyle habits of an ethnically diverse group of adolescents.

\section{Nutrition Knowledge}

The computer-based intervention resulted in a significant increase in nutrition knowledge by the third month, which was maintained through the sixth month whereas the didactic intervention resulted in a significant increase in nutrition knowledge at the third month, but this increase was not maintained through the sixth month (Table 4). These data indicate that the computer-based intervention was more effective at eliciting a prolonged increase in nutrition knowledge. For adolescents, a computer-based educational approach allows them to be actively involved in their own learning process rather than simply being a passive audience. Research indicates that an increase in knowledge does not necessarily result in improved dietary behaviors (Lytle, 2000) but can influence dietary choices in the increased nutrition knowledge is accompanied by an environment that offers concurrent positive nutrition messages. For example, a recent 
study by Conklin et al (2005) indicated that following a nutrition education intervention high schools providing nutrition information at point of the purchase resulted in healthier selections made by students. Therefore increasing knowledge can be considered the primary step in the behavior change process, but one that has to be accompanied by several other intervention components.

\section{Dietary Intake}

Neither intervention group demonstrated a significant change in kilocalorie, fat, saturated fat, fruit and vegetable or fiber intake over the course of the study (Tables 714). The $\mathrm{CBI}$ group demonstrated an increase in low-fat dairy intake and a decrease in soda intake by the third month, but returned to baseline levels by the sixth month, whereas the didactic group did not demonstrate a change over the course of the study. The control group is the only group that demonstrated a sustained increase in low-fat dairy intake over the course of the study but did not change their intake of soft drinks. These data indicate that the CBI was not more effective at eliciting a behavior change that supports healthier dietary choices.

Evidence exists that nutrition in youth affects key risk factors for chronic degenerative diseases of middle and later life, such as osteoporosis and cardiovascular disease (Dwyer, 2006). As the overweight and obesity epidemic and the resultant development of risk factors for chronic diseases continue to worsen, interventions aimed at improving dietary intake are imperative. The overall dietary habits of the population in this study suggested baseline patterns that were less than ideal. Even though the results of this study indicate the dietary intake of these adolescents may not have played a role in BMI, research indicates that the majority of risk factors for the development of chronic 
disease are influenced by diet (DeCaterina et al, 2006). The 24-hour recall and the FFQ indicated a routine intake of refined grain products, snack foods, fast foods, and pizza; these foods were often accompanied by sugary sweetened beverages. On average, the diets of these individuals were low in fruits and vegetables, low in fiber, and high in total fat and saturated fat. These results are similar to those found by the CFSII as well as by other researchers (CDC, 2002; CDC, 2000; Jacobsen, 1999; HP 2010, 2000). Soft drink consumption was also high across all three groups, while milk consumption was low.

\section{Kilocalories}

Analysis of the 24-hour recall indicated that mean energy intakes were lower than the estimated average requirement for all three groups. These are similar to the findings indicated by Skidmore and Yarnell (2004), yet few of the participants had a BMI that would be considered underweight. It is generally accepted that self-reported food intakes underestimate habitual energy intake. Although this can have an impact on the accuracy of the analysis, the dietary recall in adolescents seem to work well at least as a reference method for dietary assessments in this age group. Rothenberg et al (1998) noted that although energy intakes were underestimated on an individual basis, when comparing group data, the dietary recall method gave comparable values when validating using indirect calorimetry estimates. The groups demonstrated similar trends of underreporting and therefore it has been concluded that the 24-hour can be used for comparing intake by groups, but not as a method for assessing the adequacy of intake by individuals. Thus, energy intakes could be used as a basis of comparison in groups but not individuals. Similarly, in a study of 35 adolescents, Sjoberg et al (2003) found that dietary recalls in adolescents seem to work well at least as a reference method for dietary assessments in 
this age group. As overweight and obesity in this group continues to increase, even if these adolescents are consuming lower than recommended amounts of daily calories, it appears that minimal energy expenditure still results in a positive energy balance.

Therefore, a physical activity component that is effective is imperative to improving the risk factors in this population.

Total Fat

Although the two instruments used to assess fat intake yielded data that were equivocal, it is likely that there was not an actual significant change in total daily fat intake noted for either intervention group. The 24-hour recall indicated no significant changes in fat intake across the data collection periods, however the FFQ indicated a significant decrease in fat intake for the CBI group from baseline to post $(p<0.001)$, with an estimated $40 \mathrm{~g}$ per day decrease. The non-significant $4 \mathrm{~g}$ per day decrease in fat intake indicated by the 24 -hour recall, is more realistic than the $40 \mathrm{~g}$ per day decrease indicated by the FFQ. In this study the accuracy of the FFQ is questionable. Because it is very unlikely that such a drastic decrease occurred in the CBI group as reported by the FFQ, and the information is disputed by the 24-hour recall, it is assumed that the FFQ results were not accurate, at least for the $\mathrm{CBI}$ group at baseline. It is possible that the $\mathrm{CBI}$ participants overestimated their typical consumption of foods at baseline when completing the FFQ. Then with practice completing the tool over time, coupled with an increased knowledge of food ingredients, their responses changed when in fact their intake did not change significantly. Buzzard et al (2001) also reported some overestimation with the same FFQ, but not near the extent seen in the CBI group in the present study. Therefore, it must be assumed that despite the significant change that was 
reported using the FFQ in the CBI group, the computer-based intervention was not more effective than the TDI in eliciting a decrease in total daily fat intake.

\section{Saturated Fat}

Participants in this study reported slightly higher than the recommended percentage of energy from fat and from saturated fat. Overall, participants consumed an average of $35 \%$ of their fat as saturated. This is much greater than the recommended $10 \%$ suggested by the US Food and Nutrition Board. At the same time, they reported eating less than half of the recommended 5 servings of fruits and vegetables per day. Future interventions with groups similar to the one used in this study could benefit from focusing on community and school based initiatives to increase availability of healthier alternatives.

\section{Fruit and Vegetable Intake}

Fruit and vegetable intake was not significantly increased as a result of either intervention in this study. Intake among the current study participants suggests that only slightly more than $10 \%$ of our adolescents ate 5 or more servings of fruits and vegetables per day. The Youth Risk Behavior Survey (2004) indicated that only one in five high school students eats five or more servings of fruits and vegetables per day. NeumarkSztainer et al (2004) demonstrated that the strongest correlate of fruit and vegetable intake was the availability of these foods. It has been shown that areas of lower socioeconomic status have less access to fresh fruits and vegetables (Morland et al, 2002b). French et al. (2004) showed that increased availability of healthier alternatives resulted in increased purchases of these foods. Moreover, Haire-Joshu et al (2004) found that estimates of one's vegetable intake as a child were significantly related to exposure 
and preference for both fruits and vegetables, trying of new foods, and intake of both fruits and vegetables in adulthood. These studies suggest that interventions to increase fruit and vegetable intake in adolescents need to target socio-environmental factors such as greater availability.

Fiber

As would be expected with a low fruit and vegetable intake, fiber intake was also low across all three groups and did not change as a result of either intervention method. Average intake across all three groups was far less than the recommended intake for their age group. The recommended fiber intake for adolescents is approximately 25-30 grams per day. Although there are specific criteria set forth by the Institute of Medicine for comparing intakes to DRIs and this was not a focus of our study, the overall average fiber intake for the participants of this study was approximately 11 grams over the three data collection points. This is similar to the findings by Affenito et al (2005) who noted an average intake of 11 grams of fiber per day among adolescent girls of the same age group as used in this study. Since fiber plays a crucial role in the prevention of chronic diseases (CDC, 2003; HP 2010, 2000), efforts to improve fiber intake are needed.

\section{Low-fat Dairy and Soda}

Both intervention groups resulted in a significant change over the course of the study when considering all dairy products (Table 15). The computer-based group increased their low-fat dairy and whole fat dairy by the third month, but the change was not maintained through the sixth month. The didactic group decreased both whole and low-fat dairy consumption over the course of the study. Meanwhile, the control group also resulted in a significant increase in low-fat dairy intake by the third month and at the 
sixth month. These data indicate that the computer-based intervention was more effective at eliciting a behavior change that supports increased dairy intake than the didactic version of the intervention but other influences may play a role in dairy selection. The control group increased their low-fat dairy consumption at each data collection point. This finding may be attributed to the fact that the CON group had a larger number of students eligible for free or reduced breakfast and lunch compared to the TDI and CBI groups. Although there is no way to tell how many students enrolled in free or reduced lunches during the study, the parameters allowing for this offering by the school board was altered in the year and may explain the change during our study period. However, the policy change would have affected all three schools in the same way. Nevertheless, low-fat milk products accompany meals served at schools and the reason for the control group to increase in low-fat dairy intake over time cannot be explained by this study. Although intake of dairy products was increased, this was the only positive dietary behavior change that was demonstrated by the CON group in the study.

Overall, groups averaged drinking less than one serving of dairy per day while consuming a relatively high amount of soft drinks. The computer-based intervention did result in a significant decrease in average soda intake by the third month, which was maintained through the sixth month whereas the didactic intervention did not (Table 20). These data indicate that the computer-based intervention was more effective at eliciting a behavior change that supports decreased selection of sugary beverage consumption than the didactic version of the intervention.

The soft drink consumption (19 oz./day) in our study was similar to the national average (18-24 oz./day) (Murray, 2005; Jacobsen, 1999). Meanwhile, this high intake in 
soft drinks has been coupled with a low dairy intake, which has also been noted by several researchers (Moag-Stahlberg, 2004; Johnson et at, 2004; Jacobsen, 1999). Over the course of our study, soft drink consumption decreased in the CBI group while milk consumption increased, but the control group increased dairy and soda consumption increased simultaneously. Although a significant change in calories was not noted in any of the groups, sweetened soft drink consumption contributed an estimated $16 \%$ of these adolescents' calories at baseline. Just as studies indicate that a decrease in dairy consumption can be attributed to an increase in soft drink consumption, an increase in dairy consumption may lead to a decrease in soft drink consumption. Ludwig et al (2001) reported that the intake of soft drinks was an initial predictor of body mass and during follow-up, those with highest soft drink consumption had the highest increases in BMI. In the current study, only the CBI group demonstrated a sustained decrease in soft drink consumption. The CON group maintained high soda consumption, but was not different than the other groups, yet increased their BMI over the course of the study.

In attempting to improve dietary intake, one of the major contributors to food choice is availability. Schools continue to offer low nutrient dense foods from the vending machine and "alternative lunch lines." Positive changes often require a supportive environment that will be conducive to making the desired behavior change. The choices offered by all three schools did little to foster healthier eating habits, especially in terms of the fat and kilocalorie content. Time allotted, along with overcrowding of cafeterias and lunch lines, often leads students to gravitate towards the vending machine for lunch and snacks. 
Vending machines in these schools typically offered low nutrient dense foods. Nationwide, $98.2 \%$ of schools have vending machines, with a median of 12 machines per school (French et al, 2003). Serrano et al (2005) indicated that replacing unhealthy vending machine foods with more healthful ones could occur without a loss in profit. Moreover, French (2003) demonstrated that price reductions of $10 \%, 25 \%$, and $50 \%$ on lower fat snacks resulted in an increase in sales of $9 \%, 39 \%$, and $93 \%$, respectively compared with usual price conditions. Collaborative efforts among students, school food-service staff and research staff can be successful in implementing a large number of nutrition-related, school-wide promotional activities. These efforts can increase the sales of lower-fat foods in a la carte areas of school cafeterias and reduce consumption of foods with low nutrient density.

\section{Macronutrient Intake}

Neither intervention group resulted in a significant change in the macronutrients protein or carbohydrate, nor was a difference noted in cholesterol intake over the course of the study. In our study it appears that macronutrient intake selection did not play a role in influencing calories consumed or changes in body mass index.

\section{Micronutrient Intake}

Although a great deal of attention is given to macronutrient and energy intake in the diet of adolescents, several micronutrients are also essential to dietary metabolic balance. These nutrients were not specifically studied and the specific criteria set forth by the Institute of Medicine for comparing intakes to DRIs was not a focus of our study. The participants of this study in general appear to have consumed inadequate amounts of several important micronutrients. In fact, such intake may put these adolescents at risk 
for deficiencies. Calcium intake was approximately $50 \%$ of the DRI for this age group, which $1300 \mathrm{mg} /$ day, most likely related to a low intake of dairy products, which increases the potential risk for the development of osteoporosis later in life. Additionally, a high calcium intake has been associated with positive changes in weight maintenance and weight loss (Astrop et al, 2005; Zemel et al, 2005), while low calcium intake was found to be negatively related to children's body fat indexes (Skinner et al, 2003). Although overall iron intake was marginal, compared to the DRI of $15 \mathrm{mg} /$ day, due to its immediate health implications and impact on students' cognitive development it is an issue that should be addressed. Folate intake was also low compared to the DTI of 400 $\mathrm{mcg} /$ day. Since two of the participants that did not complete the study dropped out due to pregnancy, low intake of this micronutrient also has the potential to have serious implications for teenage pregnancy. Stang and colleagues (2000) noted that majority of adolescents in the United States do not use vitamin or mineral supplements. It was interesting to note however, that they found that adolescents who do use supplements, even on an infrequent basis, consumed diets that were more nutrient-dense than those who did not use supplements. There is a need to develop and implement future programs specifically aimed at improving the micronutrient especially iron and folic acid intakes of adolescents.

\section{Physical Activity}

The CBI resulted in a significant increase in self-reported physical activity by the third month, with a further increase by the sixth month whereas the TDI did not result in a change in physical activity over the course of the study (Table 22). These data indicate that the $\mathrm{CBI}$ was more effective at eliciting a behavior change that supports an active 
lifestyle. Increased physical activity has been shown to be an important factor for healthy weight maintenance and decreased risk factors for chronic disease (Hill, 2005; Hu et al, 2003; Kriska et al, 2004; NIH, 2004). A study by Kimm et al (2005) demonstrated that girls who were inactive during adolescence gained an average of 10-15 pounds more than active girls and experienced a three times greater gain in BMI over a 10 year period.

The lack of physical activity is playing a major role in the increases in overweight and obesity in this population (Kimm et al, 2005; Patrick et al, 2004; Tammelin et al, 2004). Student enrollment in physical education classes in Florida has decreased $46 \%$ (Macaluso, 2002) over the past 10 years. Even in those individuals that have a genetic propensity for overweight, obesity, and the development of chronic diseases, the incorporation of daily physical activity has been shown to delay or prevent the onset of overweight and obesity (Hill, 2005). Moreover, Kasa-Vubu et al (2005) concluded that for prevention strategies in youth, physical inactivity might represent a greater metabolic risk than obesity alone.

The environment is ideal for children and adolescents to consume more energy than they expend. In our study, on average, only about one in five of our participants were moderately to vigorously active on a daily basis. Because physical activity is such a significant predictor and has a beneficial effect on BMI, it remains an important topic for intervention for chronic disease prevention. The magnitude of the association with BMI to chronic disease risk factors is greater with physical activity in a combined analysis. Moreover, physical fitness has beneficial effects on numerous mediators of cardiovascular risks including overweight and obesity, so increased physical activity appears to be an ideal part of therapy. Tammelin et al (2004) has emphasized the role of 
physical activity in the transition from adolescence to adulthood finding that becoming inactive during this transition is related to overall obesity in both genders. In addition, there is speculation that physical inactivity in adolescents may be attributed to cardiovascular disease development earlier in life (Walter et al, 2005).

$B M I$

The computer-based intervention also resulted in a significant reduction in BMI by the third month, with a further decrease by the sixth month whereas the didactic intervention did not change over the course of the study (Table 6). These data indicate that the computer-based intervention utilized a more effective technique at eliciting a behavior change that supports a healthy body weight. Since the CBI group was also the only group that also increased their physical activity, and all groups had similar dietary intakes in terms of total daily calories consumed, physical activity patterns likely played a role in the observed decrease in BMI.

Many theories exist that attempt to explain the drastic increases in obesity seen among adolescents in recent years. Although the intake of nutrients required for optimal health in this population tend to be marginal and over-consumption of fats and sweets are risk factors for chronic diseases, the results of this study do not indicate that the caloric intake alone is the main culprit for the percentage of adolescents who were overweight or obese nor does it appear to have affected the BMI reduction noted in the CBI group.

According to the energy balance equation, increased caloric intake accompanied by little change in energy expenditure (physical inactivity) can account for a significant increase in weight gained per year over normal growth in this population (Ebbeling et al, 2002; Bray \& Champagne, 2005; Hancox et al, 2004). Conversely, improvements in 
dietary habits accompanied by an increase in physical activity have been shown to have positive effects in maintaining a healthy weight and improvements in BMI (Hu et al, 2004). Although there were some tangible dietary improvements made by CBI group, caloric intake, fat intake, and fruit and vegetable intake did not improve significantly in any of the groups. This indicates that there must be other factors that play a role in achieving a healthier BMI and when considering the energy balance equation, energy expenditure through physical activity is a likely culprit.

\section{Other Dietary Behaviors}

\section{Label Reading}

The computer-based intervention resulted in a significant increase in nutrition knowledge by the sixth month, whereas the didactic intervention did not change their frequency of food label reading (Table 26). These data indicate that the CBI was more effective at eliciting a behavior change that supports an improved dietary behavior. In adults increased label reading is associated with improved dietary behaviors (Neuhouser, 1999), but research regarding the correlation between adolescent food label reading and dietary choices is lacking.

\section{Meal Skipping}

Participants of our study often skipped meals. Skipping meals has been correlated with the tendency to overeat and increased intake of vending machine and high fat, high sugar, low nutrient dense foods, especially by this population. The CBI resulted in a significant decrease in number of meals skipped daily by the sixth month, whereas the didactic intervention did not change their occurrence of skipping meals (Table 21). It should be noted however, that the CBI group skipped significantly more meals at baseline 
and reduced the frequency to a similar level of the didactic and control groups. These data may indicate that the computer-based intervention may have been effective at eliciting a behavior change that supports an improved meal planning, but was not effective enough to elicit a change great enough to further improve what the didactic group was already doing.

On average, about $40 \%$ the students reported skipping a meal on the data collection days. These results are similar to those found by the CFSII (CDC, 2000). Skipping meals often contributes to poor food choices for the rest of the day (ButcherPowell, et al, 2003), and tends to increase consumption of vending machine foods such as bakery goods and snacks which can contribute to increased total dietary fat intake (Wesnes et al, 2003; Nicklas, 1998). Skipping meals is also associated with overall poorer diet quality, less variety, lower intake of fruits and vegetables and higher total sugar intake (Philippas et al, 2005).

\section{Psychosocial Factors}

Self-efficacy

Neither intervention group resulted in a significant change in self-efficacy over the course of the study (Table 23). These data indicate that the CBI was not more effective at developing what would be perceived to be an improved confidence in the ability to make dietary and physical activity behavior changes.

The average baseline self-efficacy score of approximately 2.8 across the subsections is similar to those found by Sallis et al (2004) and Rinderknect and Smith (2004) who determined baseline self-efficacy scores to be 3.0 and 2.56 respectively. Selfefficacy did not change over time among any of the groups. Although Rinderknecht and 
Smith (2004) also found that self-efficacy did not improve in a nutrition intervention in 11-18 year old adolescents, this differs from the findings of several other researchers, who have named self-efficacy as an important mediator to dietary and physical activity change (Sallis et al, 2004; Dishman et al, 2004; Wamsteker et al, 2005). One plausible explanation noted by other researchers for the lack of a change in perceived self-efficacy among adolescents is lack of time and availability of resources (safety, employment, environment, etc.) (Satia et al, 2004; Monge-Tojas et al, 2005). Schools and the retail food chains and malls that employ many of these adolescents often do not provide an environment conducive for making healthy dietary choices. Moreover, with the disappearance of physical education classes from high schools, even those that would like to engage in physical activity may find it difficult to accomplish, thus self-efficacy would remain stagnant. As with the Rinderknecht and Smith study (2004), the lack of intervention effect on self-efficacy indicates a need for a better understanding regarding the influences of the receptiveness of adolescents to lifestyle interventions. Since adolescents often find it difficult to change behaviors based upon uncertain long-term health benefits and consequences, as well as the social influences and physical environment constraints that undermine improvements in self-efficacy, these social factors that can have an impact must also be addressed. Including a component that allows for more discussion and individual feedback with a facilitator, a group discussion leader along with computer based technology may be a way to further improve health and nutrition education among this age group. Adolescents may have more confidence in their perceived ability to improve their lifestyle behaviors if they are receiving concurrent messages in other environments as well. 


\section{Social Support}

Neither intervention group resulted in a significant change in perceived social support over the course of the study (Tables 24,25 ). These data indicate that the computer-based intervention was not more effective at developing what would be perceived to be an improved social support network for the development and adoption of healthy lifestyle behaviors. Family and friends play a crucial role in the adoption and maintenance of healthy lifestyle behaviors. Participants indicated that although they did have the perception of having some support, they still had difficulty maintaining a healthy lifestyle when foods were brought into the home that were not healthy or when friends complained about time the subjects spent exercising. Their family and friends, having not been actively involved in the intervention did not seem to embrace the behaviors embarked upon by the participants. Immediately following the intervention, the groups perceived increased family and friend support in adopting healthier lifestyles, likely due to the excitement of the initiation of the program. Students in both intervention groups reported exercising together and encouraging others to engage in healthier eating habits. Simultaneously, there was an increase in perceived discouragement of the adoption of these lifestyles. This support for positive behaviors and discouragement of negative behaviors tapered off or were lost completely during the follow-up period. It is important to note that there was not an increase in the perceived discouragement of exercise by the family. Nevertheless, these results further exemplify the need for family and community involvement in future interventions and initiatives. Although most studies suggest an increased success rate with positive social support, 
negative aspects of support have been shown to be problematic to adolescents (Kelsey et al, 1997).

\section{Limitations of the Study}

There were several limitations to this study. The computer based intervention group demonstrated more positive changes, however, the treatment and the school were confounded, as there was only one treatment per school. Although comparable results were found at baseline when subjects were analyzed based on age, gender, and ethnicity, and it can therefore assumed that the school's participants were similar, there is an argument that could be made that the difference was based on the school and not the intervention. As a result, it is difficult to state with absolute certainty that the computer based method is more effective that a traditional, didactic education program.

In addition, participation was voluntary and therefore a convenience sample. Those that consented to participate may have been more accepting of or inclined toward behavior changes. The participants were all from ethnically diverse schools with greater than $70 \%$ minorities, but the schools were not necessarily comparable demographically. Additionally, the participants were primarily female, limiting the generalizability, and a larger number of subjects from a larger geographic area could be used to allow for more generalization. Also, time constraints set forth by School Board or statewide mandates did not allow for the estimated minimum of a 12-week intervention period that is recommended to detect behavior changes. Finally, many of the measures were selfreported. There is a need to generate more comprehensive tools that can be appropriately used in this population and incorporate several modes of measurement for the determination of adolescent lifestyle habits. 


\section{Conclusions}

The promotion of a healthy lifestyle is essential for the prevention of overweight and obesity and other risk factors for chronic disease. Participants in both of the intervention groups made at least some behavioral changes. In the present study, both intervention groups showed increases in knowledge, however, knowledge was accompanied by positive lifestyle changes only in the CBI group.

These data indicate that the $\mathrm{CBI}$ was more effective at eliciting a significant reduction in BMI and soda intake and a significant increase in nutrition knowledge, physical activity, and frequency of label reading over the course of the study whereas the didactic intervention did not. Of the variables in which there was a significant change, 5 of 7 of the changes were maintained at follow-up. However, the CBI was not more effective at eliciting in most of the behavior changes associated with improved dietary choices including decreased fat and saturated fat, increased fruits and vegetables, or increased fiber. Neither was it more effective in influencing other variables that can have an effect on behavior change, including social support and self-efficacy.

Finding the most appropriate method to deliver information to the widest audience possible in the most efficient and effective manner could have a huge impact on the future health of this population. This is a multi-factorial problem; progress will require a multi-factorial approach. Although the computer-based education elicited an overall greater change in behavior, no one method is the best for all students. The development of a "multi-component" model may be the best way to reach the widest range of students. However, we conclude that compared to traditional didactic teaching, computer-based nutrition and health education has greater potential to elicit changes in 
knowledge and behavior as well as promote maintenance of the behavior change over time.

\section{Applications}

Studies demonstrate that although adolescents' knowledge of healthy eating habits and consumption of fruits and vegetables is low, their attitude toward learning about healthier eating practices is favorable (Beech et al, 1999, Reynolds et al, 1999). Interventions that include nutrition, physical activity, and overall health education therefore have the potential to improve lifestyle habits and influence the future health of adolescents (Fagot Campagna et al, 2001; ADA, 2003; NIH, 2003).

There is a compelling need for innovative approaches to nutrition education. Perhaps the effectiveness that we are seeking is dependent upon a more innovative, interactive and behavioral-based way of delivering information to this population. A method that parallels the learning styles of this population along with the advances in technology seems an obvious choice for this delivery. Computer-based interventions allow for individualized strategies to promote healthy lifestyles. A more comprehensive computer assisted intervention program using an interactive, animated presentation style has the potential to elicit a greater behavior change in comparison to a traditional intervention program. Including a component that allows for discussion and individual feedback with a facilitator or group discussion leader along with computer based technologies may be a way to further improve health and nutrition education for this age group. Using innovative, practical activities coupled with a supportive environment, may enable adolescents to make positive lifestyle changes. 


\section{Future Studies}

Future studies would allow for a more complete understanding of the success a computer based healthy lifestyle education program could elicit in high school students. More schools could be used and treatments could be repeated in more than one school in order to alleviate confounding due to treatment being nested within and individual school. Also, in an effort to improve the psychosocial factors, including increased social support, increase the progression towards a readiness for change, and alleviate some of the "built environment" barriers, perhaps an additional school could be used. This third intervention group would be a multi-component group that integrated a computer-based version of the education, along with a weekly "community support group" which would jointly engage in physical activity in a safe, non-competitive environment. Making lifestyle changes not only involves the individual but also requires changes in family behaviors as well as in the social and built environment (Koplan et al, 2005). Such a group would also encourage the attendance of family and friends in order to enhance social support. A community aspect could address issues regarding availability of healthy foods. The computer-based group would likely benefit more from an intervention that was internet-based (rather than CD-ROM) that they could access at any time and send and receive support from the site as well as other participants.

A different validated Food Frequency Questionnaire may be better suitable to give more comprehensive dietary information in addition to the 24-hour recalls may be helpful. Additionally, physical activity questionnaires could be validated in all groups using pedometers (i.e. Caltrac) or heart rate monitors to ensure accurate information regarding actual physical activity is accurate. 
Evidence repeatedly confirms that many chronic diseases have their root in early childhood. Therefore, the use of more in depth techniques for identifying those at greatest risk for the development disease and beginning treatment to prevent the onset may be incorporated into this type of program. Although, childhood level of BMI was associated with adiposity and BMI-for-age was correlated with adult adiposity (Freedman et al, 2005). Other researchers have noted that although BMI is usually indicative of adiposity, it does not completely reflect body composition (Philippas et al, 2005) and other levels of assessment may also be necessary. Waist circumference is a strong independent risk factor for insulin resistance and was found to be the most powerful predictor of development of diabetes in a study by Wahrenberg et al (2005) and Han et al (2005). Those researchers found that waist circumference was a simple tool that could be used both to identify those at greatest risk for the development of chronic disease as well as exclude those who may have a high BMI but not be at risk for the development of chronic disease. Therefore, use of waist circumference in these individuals may be potentially useful for identifying those at greatest risk. Using biochemical markers for screening of hemoglobin Alc and blood lipids may potentially improve the quality of life for these adolescents, as well. The influence of nutrition and physical activity on health status and morbidity supports the need for prevention of disease and intervention strategies beginning in childhood and adolescence. 


\section{REFERENCES}

American Diabetes Association (ADA). Type 2 Diabetes in Children and Adolescents. Diabetes Care. 2003; 23, 381-389.

American Diabetes Association (ADA). American Diabetes Association. Prevention or Delay of Type 2 Diabetes. Diabetes Care. 2004; 27(1), S47-S54.

American Heart Association. Cholesterol Statistics. 2000. Available at http://216.185.102.50/cholesterol/phys.ststs.html. Retrieved August 22, 2003.

Anding JD, Kubena KS, McIntosh WA, \& O'Brien B. Blood lipids, cardiovascular fitness, obesity, and blood pressure: the presence of potential coronary heart disease risk factors in adolescents. J Am Diet Assoc. 1996; 96(3),238-42.

Bandura A. Social Foundations of Thought and Action: Asocial Cognitive Theory. Englewood Cliffs, NJ: Prentice Hall; 1986.

Bandura A. Self Efficacy In V.S. Ramachaudran (Ed.)_Encyclopedia of Human Behavior (Vol. 4, pp.71-81). New York: Academic Press. 1994.

Baranowski T, Baranowski J, Cullen KW, Marsh T, Islam N, Zakeri I, et al. Squire's Quest Dietary Outcome Evaluation Multimedia Game. Am J Prev Med. 2003, 24 (1),1293-6.

Barlow SE, Dietz WH, Klish JK, \& Trowbridge FL. Medical Evaluation of Overweight Children and Adolescents: Reports from Pediatric Nurse Practitioners and Registered Dieticians. Pediatrics. 2002; 110 (1), 222-228.

Beech BM, Rice R, Meyers L, Johnson C, \& Nicklas TA. Knowledge attitudes, and practices related to fruit and vegetable consumption of high school students. J Adol Health. 1999; 24 (4), 244-50.

Beerman KA. Computer-based Multimedia: New Directions in Teaching and Learning. J Nutr Ed Behav. 1996; 28 (1); 15-18.

Berry D. Women Maintaining Weight Loss. Diabetes. 2003, 52, 6, 569-71.

Blanchard CM, McGannon KR, Spence JC, Rhodes RE, Nehl E, Baker F, et al. Social ecological correlates of physical activity in normal weight, overweight, and obese individuals. Int J Obes. 2005; 29, 720-726.

Boreham C \& Riddoch C. The physical activity, fitness, and health of children. J Sports Sci. 2001;19 (12), 915-29. 
Bray GA \& Champagne CM. Beyond Energy Balance: Ther is more to obesity than kilocalories. J Am Diet Assoc. 2005; 105 (5 Suppl 1), S17-23.

Briggs M, Safaii S, \& Beall DL. American Dietetic Association; Society for Nutrition Education; American School Food Service Association. Position of the American Dietetic Association, Society for Nutrition Education, and American School Food Service Association--Nutrition services: an essential component of comprehensive school health programs. J Am Diet Assoc. 2003;103 (4),505-14.

Britner SL \& Pajares F. Self Efficacy Beliefs, Motivation, Race, and Gender in Middle School Science. J Women Min Sci Eng. 2001; 7, 271-285.

Brug J, Campbell M, \& van Assema, P. Differences in use and impact of computertailored dietary fat-feedback according to stage of change and education: A review of the literature. Pat Edu Coun. 1999; 36,145-156.

Brug J, Glanz K, Van Assema P, Kok G, \& Breukelen GJ. The impact of computertailored and iterative feedback on fat, fruit, and vegetable intake. Health Edu Behav. 1998; 5 (4), 517-31.

Brug J \& van Assema P. Differences in use and impact of computer-tailored dietary fatfeedback according to stage of change and education. Appetite. 2000; 34 (3), 285-93.

Budman SH. Behavioral health care dot-com and beyond: computer-mediated communications in mental health and substance abuse treatment. Am Psychol. 2000;55 (11), 1290-300.

Burnet D, Plaut A, Courtney R, \& Chin MH. A Practical Model for Preventing Type 2 Diabetes in Minority Youth. Diab Edu. 2002; 28 (5), 779-88.

Butcher-Powell LM, Bordi PL, Borja M, Cranage D, \& Cole Constance. Factors Affecting Breakfast Intake in Children. Top Clin Nutr. 2003; 18 (2), 130-35.

Buzzard IM, Stanton CA, Figuerido M, Fries EA, Nicholson R, Hogan CJ, et al. Development and reproducibility of a brief food frequency questionnaire for assessing the fat, fiber, and fruit and vegetable intakes of rural adolescents. J Am Diet Assoc. $2001 ; 101(12), 1438-46$.

Cabrinety N, Pisonero MJ, Ajram J, Armenteras A, \& Cuatrecasas JM. Lipoprotein (a) in obese children with a family history of cardiovascular disease. J Pediatr Endocrinol Metab. 2002; 15 (1),77-80. 
Center for Disease Control (CDC) National Center for Health Statistics More American Children and Teens are Overweight. News Release. Available at

http://www.cdc.gov/nchs/releases/00news/overwght99.htm Retrieved June 9, 2004.

Centers for Disease Control (CDC). CDC Funds registries for childhood diabetes. Available at www.cdc.gov/od/oc/media/pressrel/r2k1226.htm. Retrieved June 16, 2004.

Center for Disease Control and Prevention (CDC) Website. More American children and teens are overweight. Available at http://www.cdc.gov/od/oc/media/pressrel/r010312b.htm. Retrieved June 6, 2004.

Center for Disease Control and Prevention (2004). The burden of chronic disease and their risk factors: National and State Perspectives. Available at http://www.cdc.gov. Retrieved February 21, 2004.

Chumlea WC, Maynard LM, Wisemandle W, Roche AF, Guo SS, \& Siervogel RM. Childhood body composition in relation to body mass index. Pediatrics. 2001;107 (2),344-50.

Cohen J. (1988) Statistical Power Analysis for the Behavioral Sciences. $2^{\text {nd }} E d$. Hillsdale, NJ: Lawrence Erlbaum Assoc.

Conquering Diabetes. A Strategic Plan for the $21^{\text {st }}$ Century. A report of the congressionally established diabetes research working group (NIH Publication \#994398).

Contento I, Balch GI, \& Bronner YL, Theoretical frameworks of models for nutrition education. J Nutr Educ. 1995; 27, 287-290.

Cooke LJ, Wardle J, Gibson EL, Sapochnik M, Sheiham A, \& Lawson M. Demographic, familial and trait predictors of fruit and vegetable consumption by pre-school children. Public Health Nutr. 2004;7 (2),295-302.

Crocker PR, Bailey DA, Faulkner RA, Kowalski KC \& McGrath R. Measuring general levels of physical activity: preliminary evidence for the Physical Activity Questionnaire in Older Children. Med Sci Sports Ex. 1997; 29(10), 1433-9.

Cutler DM, Glaeser EL, \& Shapiro JM. Why have Americans become more obese? National Bureau of Economic Research. 2003.

Dalton S. Schools and the rising rate of overweight children- Prevention and intervention strategies. Top Clin Nutr. 2004; 19 (1), 34-40. 
De Caterina R, Zampolli A, Del Turco, Madonna SR, \& Massaro M. Nutritional mechanisms that influence cardiovascular disease. Am J Clin Nutr. 2006; 83 (2), 421S426S.

DeVries H \& Brug, J. Computer-tailored interventions motivating people to adopt health promoting behaviors: introduction to a new approach. Patient Educ Couns. 1999; 36(2), 99-105.

Di Loreto C, Fanelli C, Lucidi P, Murdolo G, De Cicco A, Parlanti N, et al. Make your diabetic patients walk. 2005; Diabetes Care. 28, 1295-1302.

Dwyer J. Starting down the right path: nutrition connections with chronic diseases of later life. Am J Clin Nutr. 2006; 83 (2), 415S-420S.

Ehtisham S, Barrett TG, \& Shaw NJ. Type 2 diabetes mellitus in UK children--an emerging problem. Diabet Med. 2000;17 (12), 867-71.

Physical Activity Does More than burn calories and improve diabetes control. Available at www.DiabetesinControl.com. Retrieved June 2, 2004.

Across the Globe 1 in 10 School Children are Overweight. Available at www.diabetesincontrol.com/issue208/item7.shtml Retrieved May 18, 2004.

Incidence of $\mathrm{CHF}$ in type 2 diabetics higher than previously estimated. Available at www.diabetesincomtrol.com Retrieved June 2, 2004.

Dietz WH. Health consequences of obesity in youth: Childhood predictors of adult disease. Pediatrics. 1998; 101, 3, 518-525.

Dishman RK, Motl RW, Saunders R, Felton G, Ward DS, Dowda M, \& Pate R. Selfefficacy partially mediates the effect of a school based physical activity intervention among adolescents girls. Prev Med. 2004; 38(5), 628-36.

Dwyer JT, Stone EJ, Yang M, Webber LS, Must A, Feldman HA, et al. Prevalence of marked overweight and obesity in a multiethnic pediatric population: findings from the Child and Adolescent Trial for Cardiovascular Health (CATCH) study. J Am Diet Assoc. 2000; 100(10), 1149-56.

Dwyer JM, Allison KR, \& Makin S. Internal structure of a measure of self-efficacy in physical activity among high school students. Soc Sci Med. 1998; 46 (9), 1175-82.

Fagot-Campagna. Appearance of type 2 diabetes in the child. Diabetes Care. 2003; 24, 767-73 
Fagot-Campagna A, Saadine J, Flegal KM, \& Beckles GL. Diabetes, impaired fasting glucose, and elevated HbA1c in US adolescents: the NHANES. Diabetes Care. 2001;24, 834-7.

Florida Department of Education (FLDOE). Student Accountability Report. Available at http://fldoe.org Retrieved June 1, 2003.

Is it time for a twinkie tax? Available at www.foodrun.com Retrieved June 6, 2004.

Foreyt JP. Behavioral Interventions. In Obesity: Impact on Cardiovascular Disease. Armonk, NY: Futura Publishing. 1999.

Freedman DS, Dietz WH, Srinivasan SR \& Berenson GS. The relation of overweight to cardiovascular risk factors among children and adolescents: The Bogalusa Heart Study. Pediatrics. 1999, 103(6), 1175-82.

Freedman DS, Khan LK, Dietz WH, Srinivasan SR, \& Berenson GS. Relationship of childhood obesity to coronary heart disease risk factors in adulthood: The Bogalusa aeart Study. Pediatrics. 2001;108 (3),712-8.

Freedman DS, Srinivisan SR, Valdez RA, Williamson DF, \& Berenson GS. Secular increases in relative weight and adiposity among children over two decades: the Bogalusa Heart Study. Pediatrics. 1997; 99, 420-425.

Freedman DS, Thornton JC, Mei Z, Wang J, Dietz WH, Pierson RN Jr, \& Horlick M. Height and adiposity among children. Obes Res. 2004;12 (5),846-53.

French SA, Story M, Neumark-Sztainer D, Fulkerson JA, \& Hannan P. Fats food restaurant use among adolescents: associations with nutrient intake, food choices and behavioral and psychosocial variables. Int J Obes Relat Metab Dis. 2001; 25(12),182333.

Galvez MP, Frieden TR, \& Landrigan PJ. Obesity in the $21^{\text {st }}$ Century. Env Health Pers. 2003; 111(13),A684-85.

Garaulet M, A. Martinez, \&Victoria F. Differences in dietary intake and activity level between normal-weight and overweight or obese adolescents. J Pediatr Gastroenterol Nutr. 2000; 30 (3), 253-8.

George V \& Shah Z. A proactive model for weight management, presented at The Annual Meeting of North American Association for the Study of Obesity, Quebec City, Canada, October 2001. Published in Med Sci Sports Exerc 33:5 Suppl, 2001. 
Giammattei J, Blix G, Marshak HH, Wollitzer AO, \& Pettitt DJ. Television watching and soft drink consumption associations with obesity in 11-13 year old schoolchildren. Arch Ped Adol Med. 2003; 157 (9), 882-6.

Giannopoulou I, Ploutz-Snyder LL, Carhart R, Weinstock RS, Fernhall B, Goulopoulou $\mathrm{S}$, et al. Exercise is required for visceral fat loss in postmenopausal women with type 2 diabetes. J Clin Endocrinol Metab. 2005; 90 (3), 1511-8.

Gibson EL, Wardle J, \& Watts CJ. Fruit and vegetable consumption, nutritional knowledge and beliefs in mothers and children. Appetite. 1998; 31(2),205-28.

Glanz K, Basil M, Maibach E, Goldberg J, \& Snyder D. Why Americans eat what they do; taste, nutrition, cost, convenience, and weight concerns as influences on food consumption. J Am Diet Assoc. 1996; 98 (10), 1118-27.

Glanz K, Lewis FM, \& Rimer BK. Health Behaviors and Health education. San Francisco: Jossey-Bass Publishers.1997.

Goran MI \& Reynolds K. Interactive multimedia for promoting physical activity (IMPACT) in children. Obes Res. 2005;13 (4),762-71.

Gower BA, Nagy TR, \& Goran MI. Visceral fat found to be metabolically unique in children. Nutrition research Newsletter. 1999; 18, 9, 15.

Gungor N, Thompson T, Sutton-Tyrrell, K, Janosky J, \& Arslanian, S. Early signs of cardiovascular disease in youth with obesity and type 2 diabetes. Diabetes Care. 2005; $28,1219-1221$.

Hancox RJ, Milne BJ, \& Poulton R. Asoociation between child and adolescent television viewing and adult health: a longitudinal cohort study. Lancet. 2004; 364, 257-262.

Healthy People 2010: National Health Promotion and Disease Prevention Objectives. Washington, DC: US Dept of Health and Human Services, Public Health Service; 2000.

Hirshler V, Gonzalez L, Bensussan T, \& Jadinsky M. Are obese girls more insulin resistant than obese boys. Diabetes 2003, 52, 6, A560-2. Can waist circumference identify children with the metabolic syndrome? Arch Pediatr Adolesc Med. 2005; 159, 740-744.

Hoelsher DM, Evans A, Parcel GS, \& Kelder SH. Designing effective nutrition interventions for adolescents. J Am Diet Assoc. 2002 Suppl. 102 (3); S52-S63.

Holcomb JD, Lira J, Kingery PM, Smith DW, Lane D, \& Goodway J. Evaluation of Jump into Action: a program to reduce the risk of non-insulin dependent diabetes mellitus in school children on the Texas-Mexico border. J Sch Health. 1998; 68 (7), 282-89. 
Huang TT, Kaur H, McCArter KS, Nazir N, Choi WS, \& Ahluwalia JS. Reading nutrition labels and fat consumption of adolescents. J Adolesc Health. 2004; 35 (5),399401.

Ivanovic D, Castro, CG, \& Ivanovic, R. Food and nutrition knowledge of school-age children's mothers from elementary to high school from different socioeconomic levels. Arch Latino-am Nutr. 1997; 47(3), 248-55.

Jackson RJ. The impact of the built environment on health: an emerging field. Am J Public Health. 2003; 93(9), 1382-1384.

Jacobsen, M. Liquid Candy. Available at www.cspinet.org/sodapop/liquid candy.htm. Retrieved January 13, 2003.

Jolliffe D. Extent of overweight among US children and adolescents from 1971 to 2000 Int J Obes Relat Metab Dis. 2004; 28(1), 4-9.

Behaviorally focused nutrition education programs for children. J Am Diet Assoc. 1999; 99 (11), 7-11.

Jago, R, Baranowski T, Baranowski JC, Thompson D, \& Greaves KA. BMI from 3-6 y of age is predicted by TV viewing and physical activity, not diet. In J Obesity. 2005; 29 (6), 557-564.

Jain A, Sherman SN, Chamberlin LA, Carter Y, Powers SW, \& Whitaker RC. Why don't low income mothers worry about their preschoolers being overweight? Pediatrics. $2001 ; 107,1138-1146$.

Jensen E. Brain-Based Learning. San Diego, CA: The Brain Store; 2000.

Johnson RK, Driscoll P, \& Goran MI. Comparison of multiple-pass 24-hour recall estimates of energy intake with total energy expenditure determined by the doubly labeled water method in young children. J Am Diet Assoc. 1996; 96 (11),1140-4.

Johnson RK, Johnson DG, Wang MQ, Smiciklas-Wright H, \& Guthrie HA. Characterizing nutrient intakes of adolescents by sociodemographic factors. J Adolesc Health. 1994; 15 (2),149-54.

Jones KL \& Dewan AK. Type 2 diabetes mellitus in adolescence: Lipid and cardiovascular risk factors. Current Diabetes Reports. 2003; 3, 255-262.

Jones LR, Sallis JF, Conway TL, Marshall SJ, \& Pelletier RL. Ethnic and gender differences in requests for and use of low/non-Fat foods in bag lunches. J Sch Health. $1999 ; 69,(8), 332-6$. 
Kasa-Vubu JZ, Lee CC, Rosenthal A, Singer K, \& Halter JB. Cardiovascular fitness and exercise as determinants of insulin resistance in post-pubertal adolescent females. J Clin Endocrinol Metab. 2005; 90(2), 849-54.

Kaufman, FR. Type 2 diabetes mellitus in children and youth: a new epidemic. J Pediatr Endocrinol Metab. 2002; 15 (Suppl 2),737-44.

Kettaneh A, Oppert JM, Heude B, Deschamps V, Borys JM, \& Charles MS. Changes in physical activity explain paradoxical relationship between baseline physical activity and adiposity changes in adolescent girls: the FLVS II study. Int J Obes. 2005; 29, 586-593.

Kimm SY, Glynn NW, Obarzanek E, Kriska AM, Daniels SR, Barton BA, et al. Relation between the changes in physical activity and body-mass index during adolescence: a multi-center longitudinal study. Lancet. 2005; 366(9482),301-7.

Kirn TF. Support, Exercise, ling-term Programs Best for Weight Loss. Fam Prac News. $2000 ; 30(6), 39-46$.

Koistinen HA, Forsgren M, Walberg-Henrikson H, \& Zierath JR. Insulin action on expression of novel adipose genes in healthy and type 2 diabetic subject. Obes Res. $2004 ; 12(1), 25-31$.

Kolasa KM, Jobe AC, \& Miller MG. Using computer technology for nutrition education and cancer prevention. Acad Med. 1996;71 (5),525-6.

Kowalski KC, Crocker PR, \& Faulkner, RA. Validation of the physical activity questionnaire for older children. Ped Ex Sci. 1997; 9, 174-186.

Krummel DA, Humphries D, \& Tessaro I. Focus groups on cardiovascular health in rural women: implications for practice. J Nutr Ed Beh. 2002; 34 (1), 38-9.

Kumar NB, Bostow DE, Schapira DV, \& Kritch KM. Efficacy of interactive, automated programmed instruction in nutrition education for cancer prevention. J Cancer Ed. 1993; $8(3), 203-11$.

Lambert L. Healthy bodies, minds, and buildings: The new physical education. Ed Leadership. 2000; 57 (6), 34-38.

Lee S, Hudson R, Kilpatrick K, Graham TE, \& Ross R. Caffeine ingestion is associated with reductions in glucose uptake independent of obesity and type 2 diabetes before and after exercise training. Diabetes Care. 2005; 28, 566-572.

Litchfeld RE, Oakland MJ, \& Anderson JA. Improving dietetics education with interactive communication technology. J Am Diet Assoc. 2000; 100 (10), 1191-4. 
Litchfield RE, Oakland MJ, \& Anderson J. Promoting and evaluating competence in online dietetics education. J Am Diet Assoc. 2002; 102,1455-1458.

Long JD \& Stevens KR. Using technology to promote self-efficacy for healthy eating in adolescents. J Nurs Scholarsh. 2004; 36 (2),134-9.

Lowry R, Kann L, Collins JL, \& Kolbe LJ. The effect of socioeconomic status on chronic disease risk behaviors among US adolescents. J Am Med Assoc. 1996; 276,792-797.

Lowry R, Galuska DA, Fulton JE, Wechsler H, Kann L, \& Collins JL. Physical activity, food choice, and weight management goals and practices among US college students. Am J Prev Med. 2000; 18(1),18-27.

Ludwig DS, Peterson KE, \& Gortmaker SL. Relation of consumption of sugar sweetened drinks and childhood obesity: a prospective observational analysis. Lancet. 2001; 357, 505-08.

Lytle L. in defense of a low-fat diet for healthy children. J Am Diet Assoc. 2000; 100(1), 39.

Macaluso CJ, Bauer UE, Deeb LC, Malone JI, Chaudhari M, Silverstein J, et al. Type 2 diabetes mellitus among Florida children and adolescents, 1994 through 1998 Public Health Rep. 2002;117, 373-9.

Masui R, Sallis JF, Berry CC, Broyles SL, \& Elder JP. The relationship between health beliefs and behaviors and dietary intake in early adolescence. J Am Diet Assoc. 2002; 102(3), 421-424.

McCarthy HD, Jarrett KV, Emmett PM, \& Rogers I. Trends in waist circumference in young British chidren: a comparative study. Int J Obes. 2005; 29(2),157-162.

McCullum C \& Achterberg CL. Food shopping and label use behavior among high school-aged adolescents. Adolescence. 1997; 32(125),181-97.

Moag-Stahlberg A. Action for healthy kids: focus on state teams- Current initiatives for sound nutrition and physical activity programs in schools. Top Clin Nutr. 2004; 19(1), 41-44.

Monge-Rojas R, Garita C, Sanchez M, \& Munoz L. Barriers and motivators for healthful eating as perceived by rural and urban Costa Rican adolescents. J Nutr Educ Behav. $2005 ; 37(1), 33-40$.

Monsen ER (Editor). Research: Successful approaches. 1992. Mexico: The American Dietetic Association. Washington DC: Library of Congress Publishing. 
Morland K, Wing S, \& Diez Roux A. The contextual effect of the local food environment on residents' diets. Am J Public Health. 2002a;92,1761-7.

Morland K, Wing S, Diez Roux A, \&Poole C. Neighborhood characteristic associated with the location of food stores and food service places. Am J Prev Med. 2002b;22(1),239.

Murray R, Frankowski B, \& Taras H. Are soft drinks the scapegoat for childhood obesity? Pediatrics. 2005; 146(5), 586-90.

Nader PR. The role of the family in obesity prevention and treatment. Ann NY Acad Sci. 1993; 29 (699), 147-53.

Neovius M, Linne Y, \& Rossner S. BMI, waist circumference and wasit-hip-ratio as diagnostic tests for fatness in adolescents. Int J Obes. 2005; 29(2),163-69.

Nestle M \& Jacobsen MF. Halting the Obesity Epidemic: A Public Health Policy Approach. Public Health Reports. 2000; 115 (1),12-24.

Neuhouser M, Kristal A, \& Patterson, R. Use of food nutrition labels associated with lower fat intake. J Am Diet Assoc. 1999; 99, 45-50, 53.

Neumark-Sztainer D, Wall M, Perry C, \& Story M. Correlates of fruit and vegetable intake among adolescents. Findings from Project EAT. Prev Med. 2003; 37(3), 198-208.

Nicklas TA, McQuarrie A, Fastnaught C, \& O'Neil CF. Efficiency of breakfast consumption patterns of ninth graders: Nutrient to cost comparisons. J Am Diet Assoc. $2002 ; 102,226-233$.

National Institute of Diabetes \& Digestive \& Kidney Diseases (2001). Weighing in on Diabetes. National Diabetes Education Program. News Release. February 21, 2001.

National Institute of Diabetes \& Digestive \& Kidney Diseases (2003). The cost of diabetes. National Diabetes Education Program News Release. February 28, 2003.

National Institute of Health (NIH). Economic burden of overweight and obesity (2003). Available at http://www.nih.gov. Retrieved January 13, 2004.

Oenema A, Brug J, and Lechner L. Web-based nutrition education: results of a randomized control trial. Health Educ Res. 2001;16 (6),647-60.

Ogden CL, Flegal KM, Carroll MD, \& Johnson CL. Prevalence and trends in overweight among US children and adolescents, 1999-2000. J Am Med Assoc. 2002; 288,1728-1732. 
Oliva P. Developing the Curriculum. $5^{\text {th }}$ edition. Boston: Little, Brown and Company. 2001.

Olshansky SJ, Passaro DJ, Hershow RC, Layden J, Carnes BA, Brody J, et al. A potential decline in life expectancy in the United States in the 21 st century. N Engl J Med. 2005; $17,352(11), 1138-45$.

Ortgega RM, Requejo AM, Lopez-Sobaler AM, Quintas ME, Andres P, Redondo MR, et al. Difference in the breakfast habits of overweight/obese and normal weight schoolchildren. Int J Vitam Nutr Res. 1998; 68 (2), 125-32.

Parcel GS, Edmundson E, Perry CL, Feldman HA, O'Hara-Tompkins N, Nader PR, et al. Measurement of self-efficacy for diet related behaviors among elementary school children. J Sch Health, 1995; 65 (1), 23-28.

Parmenter K \& Wardle J. Development of a general nutrition knowledge questionnaire for adults. Eur J Clin Nutr. 1999; 53,298-308.

Patrick K, Norman GJ, Calfas KJ, Sallis JF, Zabinski MF, Rupp J, et al. Diet, physical activity, and sedentary behaviors as risk factors for overweight in adolescence. Arch Pediatr Adolesc Med. 2004;158 (4),385-90.

Perks SM, Roemmich JN, Sandow-Pajewski M, Clark PA, Thomas E, Weltman A, et al. Energy intake estimated by the Youth-Adolescent Food Frequency Questionnaire. Am J Clin Nutr. 2000; 72,1455-60.

Powell LM, Slater S, \& Chaloupka FJ. The relationship between physical activity settings and race, ethnicity, and socioeconomic status. Evidence-Based Preventive Medicine 2004; 1 (2),135-44.

Prochaska J, Rodgers MW, \& Sallis, JE Association of Parent and Peer Support with Adolescent Physical Activity Res Quar Ex Sci. 2002; 73 (2), 206-210.

Reinehr T \& Andler W. Changes in the atherogenic risk factor profile according to degree of weight loss. Arch Dis Child. 2004; 89, 419-422.

Reynolds KD, Raczynski JM, Binkey D, Franlin FA, Duvall RC, Devane-Hart K, et al. Design of 'High-5': A school based study to promote fruit and vegetable consumption. J Cancer Educ. 1998; 13 (3), 169-77.

Reynolds KD, Baranowski T, Bishop DB, Farris RP, Brinley D, Nicklas TA, et al. Patterns in child and adolescent consumption of fruit and vegetables $\mathrm{J}$ Am Coll Nutr. 1999; 19 (3), 248-54. 
Rinderknecht K \& Smith C. Social cognitive theory in an after school nutrition intervention for urban Native American youth. J Nutr Educ Behav. 2004; 36 (6), 298304.

Robinson TN. Television viewing and childhood obesity. Pediatr Clin North Am. 2001; 48(4),1017-25.

Rodriguez R. Nutritional Genomics: What nutrition educators need to know. Oral Presentation. Society for Nutrition Educators Annual Symposium. Orlando, FL: July 26, 2005.

Rothenberg E, Bosaeus I, Lenfelt B, Landahl S, \& Steen B. Energy intake and expenditure: validation of a diet history by heart rate monitoring, activity diary and doubly labeled water. Eur J Clin Nutr. 1998; 52 (11), 832-8.

Sallis JF, Taylor WC, Dowda M, Freedson PS, \& Pate RR. Correlates of vigorous physical activity for children in grades 1 through 12: Comparing parent-reported and objectively measured physical activity. Ped Ex Sci. 2002; 14, 30-44.

Sallis JF, Grossman RM, Pinski RB, Patterson TL, \& Nader PR. The development of scales to measure social support for diet and exercise behaviors. Prev Med. 1987;16, 825836.

Sallis JF, Pinski RB, Grossman RM, Patterson TL, \& Nader PR. The development of self-efficacy scales for health-related diet and exercise behaviors. Health Ed Res. 1988; $3(3) ; 283-292$.

Sallis JF, Buono MJ, Roby JJ, Micale FG, \& Nelson JA. Seven day recall and other physical activity self-reports in children and adolescents. Med Sci Sports Exerc. 1993; $25(1), 99-108$.

Sallis JF, McKenzie TL, Conway TL, Elder JP, Prochaska JJ, Brown M, et al. Environmental interventions for eating and physical activity: a randomized control trial in middle schools. Am J Prev Med. 2003; 24 (3), 209-17.

Satia JA, Galanko JA, \& Siega-Riz AM. Eating at fast food restaurants is associated with dietary intake, demographic, psychosocial, and behavioral factors among African Americans in North Carolina. Public Health Nutr. 2004; 7 (8),1089-96.

Savoca M \& Miller C. Food selection and eating patterns: themes found among people with type 2 diabetes mellitus. J Nutr Educ. 2001; 33 (4), 224-33.

School Board of Broward County (SBBC) School Information 2002-2003. Available at http://www.browardschools.org. Retrieved July 1, 2003. 
School Board of Broward County (SBBC) School Information 2003-2004. Available at http://www.browardschools.org. Retrieved July 16, 2004.

Serrano E, Spangler J, \& Hosig K. Changes in teen beverage consumption as a result of school policy change. (Abstract) J Nutr Educ Behav. 2005; 37(Supplement 1), S45.

Shannon C, Story M, Fulkerson JA, \& French SA. Factors in the school cafeteria influencing food choices by high school students. J Sch Health. 2002; 72 (6),229-34.

Sjoberg A, Slinde F, Arviddson D, Ellegard L, Gramatovski E, Hallberg L, et al. Energy intake in Swedish adolescents: validation of diet history with doubly labeled water. Eur J Clin Nutr. 2003; 57 (12),1643-52.

Skinner H, Biscope S, Poland B, \& Goldberg E. How adolescents use technology for health information: implications for health professionals from focus group studies. J Med Internet Res. 2003; 18; 5 (4),e32.

Stang J. Assessment of nutritional status and motivation to make behavior changes among adolescents. J Am Diet Assoc. 2002; 102 (3), S52-63.

Steinberger J \& Daniels, SR. Obesity, insulin resistance, diabetes, and cardiovascular risk in children. American Heart Association Scientific Statement Circulation 2000; 107,1448-1453.

Stang J, Story MT, Harnack L, \& Neumark-Sztainer D.. Relationships between vitamin and mineral supplement use, dietary intake, and dietary adequacy among adolescents. J Am Diet Assoc. 2000;100 (8), 905-10.

Story M, Neumark-Sztainer D, \& French S. Individual and environmental influences on adolescent eating behaviors. J Am Diet Assoc. 2002; 102 (12, Suppl 3), S40-51.

Strauss RS \& Pollack HA. Epidemic increase in childhood overweight. J Am Med Assoc $2001 ; 286,2845-8$.

Sturm R. The effects of obesity, smoking, and drinking on medical problems and costs. Health Aff (Millwood). 2002; 21(2), 245-53.

Tammelin T, Laitinen J, \& Nayha S. Change in the level of physical activity from adolescence into adulthood and obesity at the age of 31 years. Int J Obes Relat Metab Disord. 2004; 28(6), 775-82.

Thakur N \& D'Amico F. Relationship of nutrition knowledge and obesity in adolescence. Fam Med. 1999; 31 (2), 122-7. 
Troiano RP, Briefel RR, Carroll MD, \& Bialostosky K Energy and fat intakes of children and adolescents in the United States; data from the National Health Examination Surveys. Am J Clin Nutr. 2000; 72, 5, 1343S-1353S.

U. S. Department of Agriculture, Agricultural Research Services, Continuing survey of food intakes by individuals (CSFII). 2000.

U.S. Department of Health and Human Services. (1999). Report of the task force on research and epidemiology and prevention of cardiovascular disease. Washington DC: National Institutes of Health, National Heart, Lung and Blood Institute.

U.S. Department of Health and Human Services. (2000). Report of the task force on research and epidemiology and prevention of cardiovascular disease. Washington DC: National Institute of Health, National Heart, Lung and Blood Institute.

Vandelanotte C, De Bourdeaudhuij I, Sallis JF, \& Brug J. Efficacy of sequential or simultaneous interactive computer-tailored interventions for increasing physical activity and decreasing fat intake. Ann Behav Med. 2005; 29(2), 138-46.

Vander Wal JS \& Thomas N. Predictors of body image dissatisfaction and disturbed eating attitudes and behaviors in African American and Hispanic girls. Eat Behav. 2004; 5(4), 291-301.

VanWormer JJ \& Boucher JL. Motivational interviewing and diet modification: a review of the evidence. Diabetes Educ. 2004; 30 (3), 404-6, 408-10, 414-6

Wahrenberg H, Hertel K, Leijonhufvud BM, Persson LG, Toft E, \& Amer P. Use of waist circumference to predict insulin resistance: retrospective study. BMJ. 2005 11; 330(7504), 1363-4. Epub 2005 Apr 15.

Wamsteker EW, Green R, Iestra J, Larsen JK, Zelissen PM, \& van Staveren WA. Obesity-related beliefs predict weight loss after an 8-week low-calorie diet. J Am Diet Assoc. 2005; 105 (3), 441-4.

Wang Y. Epidemiology of childhood obesity-methodological aspects and guidelines: what is new? Int J Obes. 2004; 28 (Suppl 3), S21-28.

Wang G \& Dietz WH. Economic burden of obesity in youths aged 6 to 17 years: 19791999. Pediatrics. 2002; 109 (5), E81-1.

Waterman R. Nutrition for Teenagers. Available at http://umanitoba.ca_health/nuteen.htm Retrieved June 2, 2003.

Webster's Dictionary. New York: Random House. 2000 
Wesnes KA, Pincock C, Richardson D, Helm G, \& Hails, S. Breakfast reduces declines in attention and memory over the morning in schoolchildren. Appetite. 2003; 41 (3), 32931.

Wild S, Roglic G, Green A, Sicree R, \& King H. Global prevalence of diabetes: estimates for the year 2000 and projections for 2030. Diabetes Care. 2004; 27(5), 1047-53.

Wilkin TJ, Voss LD, Metcalf BS, Mallam K, Jeffrey AN, Alba S, et al. Metabolic risk in early childhood: the Early Bird Study. Int J Obes. 2004; 28 (Suppl 3), S64-69.

Wright C. Children and Technology: Issues, Challenges and Opportunities Childhood Education. 2001; 78 (1), 37-43.

Youth Risk Behavior Surveillance- United States, 2003. MMWR. 2004; 53(SS-2).

Zoumas-Morse C, Rock CL, Sobo EJ, \& Neuhouser ML. Children's patterns of macronutrient intake and associations with restaurant and home eating.

J Am Diet Assoc. 2001; 101(8), 923-5. 


\section{Appendix A}

Timeline

Campus Visits 


\section{Timeline}

\begin{tabular}{|l|l|l|l|l|l|l|l|l|l|l|l|l|l|l|l|l|l|l|}
\hline $\begin{array}{l}\text { Mwareness } \\
\text { Campaign }\end{array}$ & & & & & & & & & & & & & & & & & & \\
\hline $\begin{array}{l}\text { Development } \\
\begin{array}{l}\text { Procurement } \\
\text { of Resources }\end{array}\end{array}$ \\
\hline
\end{tabular}




\section{Group 1}

\begin{tabular}{|c|c|c|}
\hline & Semester 1 & Semester 2 \\
\hline Awareness & 28-May & \\
\hline Recruitment & 16-Aug & \\
\hline & 17-Aug & \\
\hline & 18-Aug & \\
\hline & 26-Aug & \\
\hline & 31-Aug & \\
\hline Screening & 2-Sep & \\
\hline & 3-Sep & \\
\hline & 7-Sep & \\
\hline & 8-Sep & \\
\hline & 10-Sep & \\
\hline Baseline a & 14-Sep & \\
\hline $\begin{array}{l}\text { Make up } \\
\text { Baseline a }\end{array}$ & 17-Sep & \\
\hline Baseline b & 28-Sep & \\
\hline $\begin{array}{l}\text { Make up } \\
\text { Baseline b }\end{array}$ & 7-Oct & \\
\hline Session 1 & 11-Oct & \\
\hline Session 2 & 18-Oct & \\
\hline$\frac{\text { Make up }}{\text { Session } 1 \&}$ & & \\
\hline 2 & 22-Oct & \\
\hline Session 3 & 25-Oct & \\
\hline Session 4 & 1-Nov & \\
\hline$\frac{\text { Make up }}{\text { Session } 3 \text { \& }}$ & & \\
\hline 4 & 5-Nov & \\
\hline Session 5 & 8-Nov & \\
\hline $\begin{array}{l}\text { Make up } \\
\text { Sessions }\end{array}$ & 15-Nov & \\
\hline \begin{tabular}{|l} 
Make up \\
Sessions
\end{tabular} & 16-Nov & \\
\hline Post-Testa & 30-Nov & \\
\hline Post Test b & 7-Dec & \\
\hline $\begin{array}{l}\text { Make Up } \\
\text { Post Test }\end{array}$ & 16-Dec & \\
\hline $\begin{array}{l}\text { Make Up } \\
\text { Post Test } \\
\end{array}$ & & 11-Jan \\
\hline Follow Up a & & 29-Mar \\
\hline Follow Up $b$ & & 7-Apr \\
\hline \begin{tabular}{|l|} 
Make Up \\
Follow Up \\
\end{tabular} & & 19-Apr \\
\hline$\frac{\text { Make Up }}{\text { Follow Up }}$ & & $21-A$ \\
\hline
\end{tabular}

Group 2

Semester 1 Semester 2

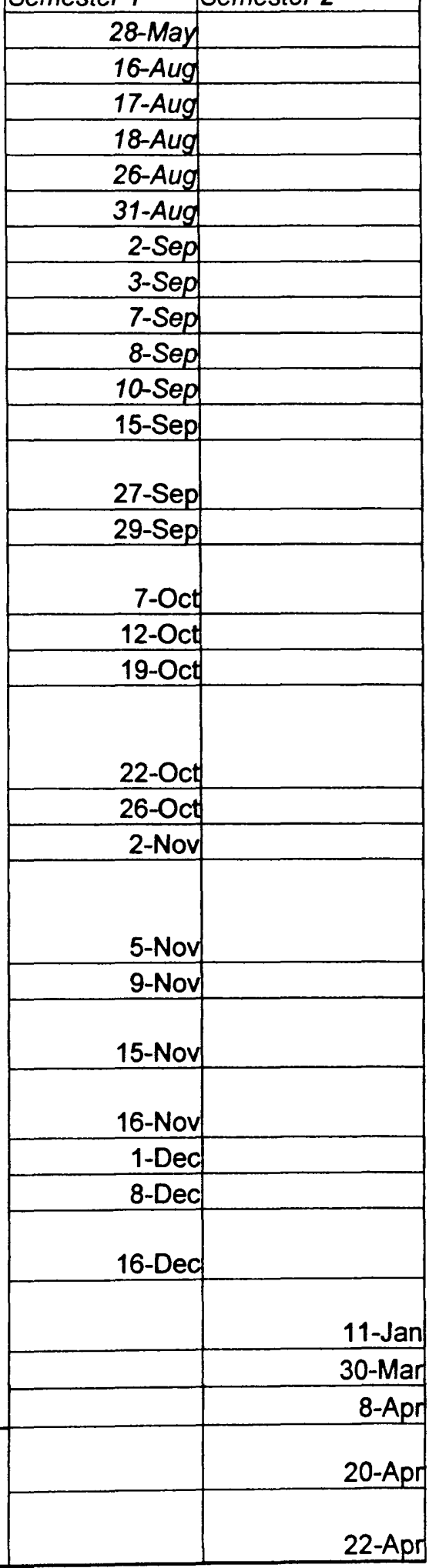




\begin{tabular}{|c|c|c|c|c|}
\hline \multicolumn{3}{|l|}{ Group 1} & \multicolumn{2}{|l|}{ Group 2} \\
\hline & Semester 1 & Semester 2 & Semester 1 & Semester 2 \\
\hline Awareness & 3-Jun & & 3-Jun & \\
\hline Recruitment & 19-Aug & & 19-Aug & \\
\hline & 20-Aug & & 20-Aug & \\
\hline & 23-Aug & & 23-Aug & \\
\hline & 30-Aug & & 30-Aug & \\
\hline Screening & 2-Sep & & 2-Sep & \\
\hline & 3-Sep & & 3-Sep & \\
\hline & $8-\mathrm{Sep}$ & & $8-S e p$ & \\
\hline & 9-Sep & & 9-Sep & \\
\hline & $10-\operatorname{Sep}$ & & $10-\operatorname{sep}$ & \\
\hline Baseline a & 21-Sep & & 22-Sep & \\
\hline Make up Baseline a & 27-Sep & & $27-$ Sep & \\
\hline Baseline $b$ & $30-S e p$ & & 1-Oct & \\
\hline Make up Baseline b & 1-Oct & & $1-O c t$ & \\
\hline Session 1 & $20-0 c t$ & & $21-O c t$ & \\
\hline Session 2 & 27-Oct & & 28-Oct & \\
\hline Make up Session 1 \& 2 & 29-Oct & & 29-Oct & \\
\hline Session 3 & 3-Nov & & 4-Nov & \\
\hline Session 4 & 10-Nov & & $12-$ Nov & \\
\hline Make up Session $3 \& 4$ & 5-Nov & & 5-Nov & \\
\hline Session 5 & 17-Nov & & 18-Nov & \\
\hline Make up Sessions & 19-Nov & & 19-Nov & \\
\hline Make up Sessions & 22-Nov & & 22-Nov & \\
\hline Post-Testa & 2-Dec & & 3-Dec & \\
\hline Post Test b & 9-Dec & & 10-Dec & \\
\hline Make Up Post Test & 17-Dec & & 17-Dec & \\
\hline Make Up Post Test & & 12-Jan & & 12-Jan \\
\hline Follow Up a & & 31-Mar & & 1-Apr \\
\hline Follow Up b & & 12-Apr & & 13-Apr \\
\hline Make Up Follow Up & & 21-Apr & & 21-Apr \\
\hline Make Up Follow Up & & 22-Apr & & 22-Apr \\
\hline
\end{tabular}


SCHOOL 3

\begin{tabular}{|c|c|c|c|c|}
\hline \multicolumn{3}{|l|}{ Group 1} & \multicolumn{2}{|l|}{ Group 2} \\
\hline & Semester 1 & Semester 2 & Semester 1 & Semester 2 \\
\hline Awareness & \begin{tabular}{|r|} 
4-Jun \\
\end{tabular} & & \begin{tabular}{|r|} 
4-Jun \\
\end{tabular} & \\
\hline Recruitment & 24-Aug & & 24-Aug & \\
\hline & 25-Aug & & 25-Aug & \\
\hline & 27-Aug & & 27-Aug & \\
\hline & 1-Sep & & 1-Sep & \\
\hline Screening & 2-Sep & & 2-Sep & \\
\hline & 3-Sep & & 3-Sep & \\
\hline & 9-Sep & & 9-Sep & \\
\hline & 10-Sep & & 10-Sep & \\
\hline & 13-Sep & & 13-Sep & \\
\hline Baseline a & 23-Sep & & 24-Sep & \\
\hline $\begin{array}{l}\text { Make up } \\
\text { Baseline a }\end{array}$ & & & & \\
\hline Baseline b & 5-Oct & & 6-Oct & \\
\hline $\begin{array}{l}\text { Make up } \\
\text { Baseline b }\end{array}$ & & & & \\
\hline Post-Testa & 23-Nov & & 24-Nov & \\
\hline Post Test $b$ & 14-Dec & & 14-Dec & \\
\hline $\begin{array}{l}\text { Make Up } \\
\text { Post Test }\end{array}$ & & & & \\
\hline$\frac{\text { Make Up }}{\text { Post Test }}$ & & 13-Jan & & 13-Jan \\
\hline Follow Up a & & 5-Apr & & 6-Apr \\
\hline Follow Up b & & 14-Apr & & 15-Apr \\
\hline $\begin{array}{l}\text { Make Up } \\
\text { Follow Up }\end{array}$ & & 26-Apr & & 26-Apr \\
\hline$\frac{\text { Make Up }}{\text { Follow Up }}$ & & 27-Apr & & 27-Apr \\
\hline
\end{tabular}




\section{Appendix B}

Lesson Plans

Study Guides 
Introduction

I. Adolescent Nutrition

a. Despite teens' familiarity with nutrition information, many don't eat enough of some foods, including fruits and vegetables.

b. Most teens need to eat foods that are lower in fat and sugars.

c. Teens in the US participate in less physical activity than ever before.

II. Understanding Adolescent Nutrition

a. There is a high risk of following vitamin and mineral deficiency (especially in girls).

- Iron

- $\quad$ Folate

- Calcium

- Vitamin A

b. More than $30 \%$ of teens are currently overweight or obese, and this number is increasing.

c. Obese adolescents enter the job market at lower salaries.

III. Eating breakfast

IV. Dietary Guidelines

a. Experts have made it easier for you to plan and eat nutritious meals using the "DIETARY GUIDELINES" (OVERHEAD)

i. These help you guide your food selections

ii. They are based on 3 principles

1. AIM for fitness

2. BUILD a healthy base

3. CHOOSE sensibly

V. Food Guide Pyramid

a. The Food Guide Pyramid is a graphic illustration that can help you meet the Dietary Guidelines. (OVERHEAD)

i. It is used to show variety, moderation, and proportion of a wellbalanced diet.

VI. How much is a Serving? OVERHEAD

VII. Breads, Cereals, Rice and Pasta

a. Bread, cereal, rice and pasta are the foundation of the pyramid

VIII. Vegetables and Fruits

a. Tips to Increase Your Fruits and Veggies (OVERHEAD)

IX. Meat

a. Foods in this group include beef, pork, fish, shellfish, veal, eggs, poultry, dried beans and nuts.

b. These foods are valuable sources of protein, B-vitamins, and iron.

$X$. Lowering the Fat in Your Diet (OVERHEAD)

XI. Choices -More or Less (OVERHEAD)

XII. Milk, Yogurt, and Cheese

a. Milk and milk products are our best source of calcium.

XIII. Fats, Oils and Sweets

a. These are foods that should be eaten sparingly - not too much each day! 
XIV. The road to dietary improvement. (OVERHEAD)

a. You have special nutritional needs.

b. You have to take responsibility for which foods and how much of those foods you eat.

c. Breakfast is important.

d. Snacks fit in but choose wisely for better nutrition.

e. Set realistic goals for dietary improvements.

XV. Snacking

XVI. Summary 
I. Food Guide Pyramid Review:

a. Most of our calories should come from bread, cereals, pasta, and rice.

i. Start at the bottom of the Food Guide Pyramid, by eating 6 to 11 servings of foods from grain products (breads, cereals, pasta and rice), 3-5 servings of vegetables and 2-4 servings of fruits each day.

ii. These foods provide carbohydrates.

II. Kinds of Carbohydrates:

b. There are 2 main kinds of carbohydrates:

a. simple

a. Example: table sugar

b. Simple sugars are naturally found in many foods; but, they are also often added to foods.

c. Added sugars increase the number of calories.

d. We need to reduce our intake of foods or beverages with a lot of sugar added.

i. These include sweets and beverages (sweet tea, soda, fruit drinks, sports drinks) that have little or no nutritive value and give primarily calories.

e. Instead, we need to eat more complex carbohydrates and drink fruit juice.

f. OVERHEAD - WHICH IS HEALTHIER?

b. Complex

a. Examples: starches and dietary fibers.

b. All starchy foods are plant foods.

c. Major food sources of starch include:

i. grains (breads, cereals, rice)

ii. legumes (beans and peas),

iii. potatoes and sweet potatoes.

iv. Fruits and vegetables have starch as well as fiber.

d. Fiber

i. Fiber is the non-digested portion of plant foods

ii. Fiber is important in maintenance of your lower gut

1. A diet high in fiber helps relieve constipation

iii. Fiber also helps to improve the health of your heart

1. A diet high in fiber helps lower cholesterol

iv. Fiber also helps reduce the risks of cancer

c. What about the Atkin's Diet?

a. Much of the media and diets are focusing on reducing carbs.

b. Diets such as Atkins and South Beach suggest that the reduction or elimination of carbs is the key to losing weight.

c. What are the facts and does it really work? 
i. Carbohydrates are our body's favorite source of energy.

ii. We can get energy from protein and fat, but protein is better used for building our body and keeping it working well, \& eating extra fat can be unhealthy.

iii. Elimination of carbohydrates from the body forces the body to rely on fat and protein.

iv. Some parts of the body have to have carbohydrate to function properly (eyes, brain).

1. Without adequate carbs other health issues may arise.

2. It can be detrimental to performance of an athlete!

3. Also, high fat/protein diets increase your risks for some diseases.

4. High protein diets can also increase your risk of becoming dehydrated.

v. Carbohydrates are NOT the enemy. Weight gain is primarily due to eating more calories than you burnnot necessarily the result of the type of calories that you eat.

1. Carbohydrates are the foundation of a healthy diet.

2. Carbohydrate foods should be chosen wisely!

d. What Counts as a Carbohydrate Serving?- OVERHEAD

e. Easy Ways to Get More Carbs -OVERHEAD

III. Protein Content of Foods

a. Protein Content Foods OVERHEAD

c. Function of Proteins - OVERHEAD

d. Protein \& Health

i. Foods high in animal protein are often also high in saturated fat and cholesterol

ii. Increase risk of heart disease

iii. Increased saturated fat and cholesterol increases risk of heart disease

iv. Osteoporosis and bone fractures

1. High protein leads to more calcium excretion

2. Kidney disease - high protein diets make the kidneys work harder

e. Protein Requirements- OVERHEAD

f. Vegetarians

i. Although plant proteins are considered low protein foods, vegetarians can still easily meet their protein requirement. 
IV. Fats

ii. Eating a variety of plant products results in complementary protein status.

iii. In other words, what one plant protein may be lacking can be eaten with another plant protein that provide that amino acid.

g. Fat is a substance found naturally in many foods.

i. Fats added during preparation can add lots of unwanted calories.

ii. The body needs some fat for survival

iii. Not all fat is bad; but fat should be consumed in moderation!

h. Why we need fat

i. Energy

1. Fat is 1 of the 3 key nutrients that gives calories.

2. Fat gives 9 calories per gram, and both carbohydrate and protein give 4 calories each. A little fat goes a long way!

ii. Carries Fat-Soluble Vitamins

1. Some fats and oils are important sources of vitamins A, $\mathrm{D}, \mathrm{E}, \& \mathrm{~K}$ which are essential for proper growth and development.

iii. Insulates and Protects Your Body

1. Fat surrounds and protects your organs and tissues. It also help you regulate your body temperature.

i. Why too much fat is bad?

i. Since fat has more calories than protein and carbohydrate, excess fat consumption can lead to overweight.

ii. Excess fat and "bad" cholesterol are transported in the blood.

j. What is cholesterol?

i. Cholesterol is fat-like substance found only in animal products. Plants don't have cholesterol!

ii. Excess levels in the blood can block arteries and lead to heart disease.

iii. There are 2 types of cholesterol

1. LDL - bad cholesterol

2. HDL - good cholesterol

k. Cholesterol OVERHEAD (Cheerios)

1. Kinds of Fat

i. To better understand the types of fat, we will consider the following family:

1. King Sat (Saturated) - OVERHEAD

2. Queen Mama Mono (Monounsaturated) - OVERHEAD

3. Princess Poly (Polyunsaturated) - OVERHEAD

m. Dietary Guidelines

i. Choose a diet that provides no more than $30 \%$ of total calories from fat. 
ii. For example, at 2,000 calories per day, the suggested upper limit of calories from fat is about 600 calories. 65 grams of fat per day)

iii. Reducing saturated fat will help you lower your bad cholesterol level.

1. Take in less than $180 \mathrm{kcal}$ per day of saturated fat or 20 grams per day

2. Also, cutting back on fat can help consume fewer calories.

iv. Did you know that:

1. Exercise can Increase HDL (good cholesterol) levels

V. Vitamins

v. Which is "healthier"? - OVERHEAD

a. Vitamins are nutrients required by the body in small amounts to perform specific functions.

i. Water-Soluble (B's and C)

1. Many of the B vitamins are involved in conversion of foods to energy

2. Vitamin $\mathrm{C}$ is an antioxidant (protects the body from stress) and is an important part of collagen synthesis

ii. Fat-Soluble (A,D,E,K)

b. Vitamins OVERHEAD

c. Vitamins \& Disease

i. Taking vitamin supplements will not improve performance (athletic, academic, etc.) directly, but will prevent a deficiency.

ii. Some vitamins called "anti-oxidants" may decrease diseases such as cancer and heart disease.

VI. Minerals

1. $\mathrm{C}$ and $\mathrm{E}$ are anti-oxidant vitamins

a. There are many minerals that are important to the body. The following are of particular concern in adolescents:

i. Sodium- important for water balance although elevated levels can cause high blood pressure

ii. Calcium - important for maintaining strength of your bones and teeth

iii. Iron - important in the production of red blood cells

b. How much Calcium and Iron do I need? - OVERHEAD

c. Pass on the salt

i. Salt is a mineral that is often used in excess

ii. Processed foods are often very high in salt (sodium)

iii. Attempt to limit your sodium to $2000-4000 \mathrm{mg}$ !

iv. A diet high in sodium can lead to high blood pressure.

VII. Importance of Proper Hydration - OVERHEAD

a. Functions of water-OVERHEAD

b. Water Balance 
i. Balance

1. Intake should equal Losses

2. By the time you are thirsty you have already lost $2 \%$ of your body water

3. Losses

4. It is Recommended that you drink AT LEAST 8 cups a day

c. Staying hydrated - OVERHEAD

d. Effect of Dehydration on Physical Performance- OVERHEAD 
I. Obesity is the biggest nutritional problem of teens in the US.

a. Due to poor diets and lack of physical activity, it's estimated that $16-33 \%$ of teens in the US are overweight or obese.

b. Overweight teens are more likely to develop for heart disease and type 2 diabetes.

II. Overweight Adolescence

a. When you are overweight as a child, you may increase the size of your fat cells (more storage) and also increase the number of fat cells you have.

i. Therefore, it may be more difficult to lose weight than a person who has maintained a more normal weight.

ii. As an adult, it is more difficult to lose weight if you are overweight or obese as an adolescent

b. Fat cells in adolescence - OVERHEAD

c. Body Mass Index or BMI is a tool for indicating weight status. It is a measure of weight for height.

i. BMI -OVERHEAD

ii. BMI usually correlates with body fat. The relation between fatness and BMI differs with age and gender.

1. However, people with a lot of muscle mass may have a high BMI and low body fat

2. Increased BMI often increases risks of developing diabetes and heart disease.

III. What is Diabetes?

a. Diabetes is a disease that impairs the body's ability to obtain energy from food.

i. The hormone insulin signals the cells of the body to allow glucosethe body's main energy source- to enter.

ii. People with diabetes either cannot make insulin or the body cannot use the insulin the body makes.

iii. Insulin - OVERHEAD

b. Two types of Diabetes

i. Type 1

1. Body does not make insulin

2. Blood glucose is elevated

3. Usually early diagnosis due to immediate onset

4. $5 \%$ of diabetes cases

ii. Type 2

1. Body cannot use insulin

2. Blood glucose is elevated

3. Often associated with being overweight

4. Excess fat prevents insulin from working properly

5. Usually occurs gradually 
6. $95 \%$ of diabetes cases

iii. The most common feature of both types is elevated blood sugar.

1. This is also the main factor in diagnosis of the disease

2. The age group 12-19 is described as the age group at highest risk for developing type 2 diabetes.

3. Onset of diabetes in adolescence can lead to severe complications later in life.

c. Diabetes Complications - OVERHEAD

IV. What is Heart Disease?

a. Heart disease goes by many names: coronary heart disease, coronary artery disease, atherosclerosis, ischemic heart disease and cardiovascular disease, to name a few.

b. Heart disease is the leading cause of death in the US.

c. Development of Heart Disease - OVERHEAD

i. Persons with diabetes are $3 \mathrm{X}$ more likely to develop heart disease than non-diabetic individuals.

ii. $80 \%$ of deaths of patients diagnosed with type 2 diabetes results from complications of heart disease.

iii. Accumulation of fat around the abdomen is a risk factor for heart disease and may be at risk for type 2 diabetes.

d. Diet and Heart Disease - OVERHEAD

e. Heart Disease Risk Factors - OVERHEAD

f. Physical Activity \& Heart Disease - OVERHEAD 
You Are in Charge!

I. Take Control of Your Health

a. Are you taking care of your body?

a. Self-awareness

b. Does your diet need improvement?

b. Are you meeting the dietary recommendations?

c. Are you participating in regular physical activity?

II. Responsibility

a. You are responsible for the dietary and physical activity choices that you make.

b. Health problems associated with poor diet and physical inactivity

a. Increased weight gain

b. Heart disease

c. Diabetes

d. High blood pressure

c. You have the power to improve your health

a. This is important to your future health status

b. You always have choices

c. Set reasonable expectations

d. Get rid of excuses

e. Engage in activities that are meaningful to you

d. Changing Your Behavior

a. There are several things that may affect your ability to change unhealthy behaviors - OVERHEAD

III. Social support

a. It is easier to make dietary changes when you have support and encouragement from your friends and family!

b. Social Influence

a. Explain your interest in improving health to your friends and family

b. Invite friends and family members to eat healthy and exercise with you

c. Select activities and foods that may be enjoyable to your family and friends so that they too may participate

III. Self-efficacy

a. You have to believe that you are capable of making lifestyle changes

i. Maintain a positive attitude

ii. Communication

iii. Ask for the support of your family and friends.

iv. Listen to suggestions from others

v. Give and accept feedback

vi. When dining out, ask for low-fat versions.

b. Network

i. Develop new friendships with people that have similar goals

ii. Join a group or club such as a walking group or inline skating club.

c. Stay Motivated 
i. Monitor your achievement by setting weekly dietary and physical activity goals

ii. Reward yourself for each goal that your reach

iii. Encourage others to stay motivated

iv. Improve Skill

1. The longer you maintain an activity the better you will become at it.

d. Read food labels

i. You will become better at estimating calorie intake

ii. Practice money saving shopping tips clip coupons, look for sales, compare prices

e. Be active

i. The more active you are on a regular basis, the easier participating in physical activities will become

f. Keys to good health

i. The better your diet is the better you will feel.

ii. Regular physical activity increases your energy

IV. Team work!

a. Don't go at it alone.

b. Talk to your friends and family about the changes that you want to make.

c. Encourage friends and family to improve their dietary and physical activity patterns too.

d. Ask for help in meal preparation and time management that will make it easier to make lifestyle changes.

V. Setting Goals

a. A goal is the result toward which effort is directed.

b. Setting goals may help you achieve success in changing unhealthy behaviors.

c. Writing Goals - OVERHEAD

i. Priorities

1. What activities will be tried?

a. Examples: - OVERHEAD

d. Barriers

i. Making behavior changes can be difficult due to several barriers

1. Time

2. Money

3. Lack of support

4. Lack of availability of resources

5. But, taking responsibility for your actions, making good decisions regarding your health, along with a sincere dedication to succeed can make overcoming these barriers a little easier

VI. Time Management - OVERHEAD

VII. Reasonable Expectations - OVERHEAD

VIII. Energy Balance Equation - OVERHEAD 
IX. Setting Goals for Healthy Weight Management -OVERHEAD

a. Setting Physical Activity Goals - OVERHEAD

b. Setting Dietary Goals- OVERHEAD

$\mathrm{X}$. Body Weight - OVERHEAD

a. Yo-Yo Dieting

i. Healthy weight loss usually involves loss of $75 \%$ fat and $25 \%$ muscle

ii. Unhealthy weight loss, such as going on a very low calorie diet (less than 1200 calories per day) involves a loss of $50 \%$ fat and $50 \%$ muscle

iii. In addition, the body becomes more efficient at food storage

iv. When you resume normal eating, weight gain often occurs much quicker

v. This is referred to as yo-yo dieting and can be very unhealthy

XI. Healthy Weight

a. Body Mass Index (BMI) is used as an estimate for healthy weight status

Underweight

Healthy Weight

Overweight

Obese

Morbidly obese $<18.5$

$18.5-24.9$

$25-29.9$

30-39.9

$>40$

b. It is calculated by comparing your height to your weight

c. Interpret carefully as it does not take into account large amounts of muscle mass

d. A muscular athlete may be considered obese despite a low body fat percentage.

XII. Health Risks

a. There are health risks on both sides of the healthy weight range

i. Underweight increases chances of infection, results in fatigue, and may lead to vitamin and mineral deficiencies

ii. Overweight increases your risk of heart disease, diabetes, and some cancers

iii. Set your goals for a healthy weight!

XIII. Eating Plan for Weight Management - OVERHEAD

XIV. Benefits of Physical Activity for Weight Management - OVERHEAD

XV. Reward Yourself - OVERHEAD 
I. The key to healthy eating is balance, variety, and moderation: OVERHEAD

II. Planning Principles- OVERHEAD

a. Plan Before Shopping

i. Planning is the key to saving money.

ii. Using the meals you've planned, make a shopping list.

iii. Once you've figured out what you have to spend, begin planning meals.

iv. Keep the Food Guide Pyramid in mind as you plan your meals and make your shopping list.

v. Also clip any coupons for foods or items that you would normally use.

b. Shop Smart

i. Once you've finished planning, it's time to learn to shop smart. Always shop with a list.

ii. Clip coupons only for the things you need.

iii. Don't shop when you're hungry. Hungry shoppers buy more than they intended to buy.

iv. Go down only the aisles that have something on your list.

v. Be aware of end-of-aisle displays. These are not always moneysaving deals.

III. Buying Products

a. Buying Grain Products OVERHEAD

b. Buying Fruits \& Vegetables - OVERHEAD

c. Buying Meats - OVERHEAD

IV. "Nutrition Facts" Label

a. Reading the Label - OVERHEAD

b. Lowering Calories and Fat- OVERHEAD

c. Fat Matters, but Calories Count- OVERHEAD

d. Reduced Fat/Fat free vs. Regular- OVERHEAD

V. Food Preparation- What to Do

a. Certain ingredients can add unwanted calories and fat to otherwise low-fat meals

b. Low-Fat Cooking Methods- OVERHEAD

VI. Menu Planning Tips- OVERHEAD

VII. Fast Food

a. Fast food is especially popular among teens

b. Eating fast foods is associated with a higher intake of calories and fat and lower intake of vitamins and minerals

c. Eating Smart on the GO! - OVERHEAD

d. General Tips for Dining Out- OVERHEAD

e. Specific Tips for Healthy Choices- OVERHEAD

VIII. Eating

a. Eating slowly will help you eat less 
b. It takes 15 minutes for the brain to realize that you are full

c. Eat more fruits and vegetables

i. They give you the feeling of fullness and have other benefits as well

d. Drink plenty of water and non-caloric beverages

i. It will help you feel full and keep you hydrated

e. Don't skip meals!

i. Set an eating schedule so you don't overeat later.

IX. Physical Activity - OVERHEAD

a. Incorporating Physical Activity into your Daily Life - OVERHEAD 
Section I: Introduction

\section{Session 1: Introduction to Adolescent Health}

\section{A. Dietary Health Risks}

1. What vitamin and mineral deficiencies are common among teens

(especially girls)?

2. Name some advantages of eating breakfast in the morning?

Section II: Dietary Guidelines

1. What are the 3 principles that the dietary guidelines are based upon?

Section III: Food Guide Pyramid

a. Bread, Cereals, rice, and pasta (6-11 servings)

1. Why are bread, cereals, rice, and pasta the foundation of a wellbalanced diet?

2. What are some nutrients found in foods in this group?

3. What is a tip that you may use to save money on bread, cereal, rice, and pasta products in order to get your 6-11 servings per day?

b. Fruits (2-4 servings) and vegetables (3-5 servings)

4. What are some key nutrients found in fruits and vegetables?

5. What are 2 tips that you may use to increase your fruit and vegetable intake?

c. Meat

6. What nutrients are found in meat?

7. How much is a serving of meat?

8. What changes in your meat selections could help you to lower the fat in your diet?

d. Milk, cheese, and yogurt

9. What is the best source of calcium in the diet?

10. What happens if you don't get enough calcium when you are young?

11. How can you get the required amounts of calcium without getting extra fat?

12. How much calcium should you be consuming each day?

e. Fats, oils, and sweets

13. Name 3 ways that you could lower the fat in your diet.
i.
ii.
iii.

Section IV: Physical Activity

1. Name at least 3 benefits of physical activity.
a. .
c.
b. .

2. What are the 2 types of physical activity and how are they different?

3. Name some ways that you could increase your daily physical activity.
a. .
b. . 
A. Carbohydrates

\section{Study Guide Questions \\ Session 2: Overview of the Nutrients}

1. What nutrient should make up the majority of the calories of our diet?

2. What do carbohydrates do in the body?

3. What food groups contain good sources of carbohydrates?

4. What are the 2 types of carbohydrates and which one do we need more of?

5. In general, are carbohydrates fattening?

6. Name some ways that you could get more carbohydrates in your diet?

B. Protein

7. Name some foods that are high in protein, but low in fat.

i.

ii.

8. Do plant products have protein in them? If so how are they different from animal protein?

9. What are some good sources of protein?

10. What are legumes?

\section{Fat}

11. Name some reasons why fat is an important part of the diet?

i.

ii.

iii.

iv.

12. What is the unhealthy type of fat? Name 2 examples of foods that contains this fat.

i.

ii.

13. What is the type of fat that is the healthiest? Name 2 examples of foods that contain this fat.

i.

ii.

14. What are some concerns with eating a high fat diet?

i.

ii.

iii.

15. What are ways that you could easily lower the fat content of your diet?

i.

ii.

iii.

16. Which is healthier: butter or margarine? 
17. Why do we need vitamins?

D. Vitamins

18. What are the 2 categories of vitamins and how are they different?

19. Explain the function of the following vitamins and name a food that is a good source of that vitamin:

\begin{tabular}{|l|l|l|}
\hline Vitamin & Function & Source \\
\hline Vitamin A & & \\
\hline Vitamin C & & \\
\hline Vitamin D & & \\
\hline Vitamin E & & \\
\hline Vitamin K & & \\
\hline B Vitamins & & \\
\hline
\end{tabular}

20. How could you get all of your vitamins without taking a vitamin supplement? E. Minerals

21. Why are minerals important to your body?

22. Name 3 major minerals that are important in your body?

i.

ii.

iii.

23. What is the function of the following minerals?

\begin{tabular}{|l|l|}
\hline Mineral & Function \\
\hline Iron & \\
\hline Calcium & \\
\hline Sodium & \\
\hline
\end{tabular}

25. What can you do to be sure that you get the kinds and amounts of minerals that you need each day?

26. Name some functions of water.

\section{F. Water}

i.

ii.

iii.

27. Name some tips that you think would be reasonable to increase your water intake each day.

i.

ii.

28. How can you assess your own hydration status? 


\section{Study Guide Questions}

Obesity

\section{Session 3: Nutrition for Life}

1. What is the most prevalent nutritional disease of children and adolescents in the US?

2. How may being overweight in adolescence affect your ability to maintain a healthy weight as an adult?

Healthy Weight

3. What is BMI?

4. What measurements does BMI come from?

5. What is the normal range for BMI?

6. What is your BMI?

7. How does BMI relate to health status?

8. Why should BMI values be carefully interpreted?

Type 2 Diabetes

9. What does insulin do?

10. How does overweight and obesity affect insulin function?

11. What is the most common type of diabetes?

12. What age group is at highest risk for the development of type 2 diabetes?

13. What are some complications of type 2 diabetes?

Heart Disease

14. What is the leading cause of death among Americans?

15. What are some risk factors associated with heart disease?

16. How can you lower your risks of heart disease? 


\section{Study Guide Questions}

Session 4: Taking Responsibility for Your Health

1. Name some health problems associated with poor diet and physical inactivity.

i.

ii.

iii.

2. It is easier to make dietary changes when you have friends and family! from your

3. is believing that you have the ability $\mathrm{t}$ make behavior changes. i.

ii.

iii.

4. Name some things that can affect your ability to make behavior changes.

i.

ii.

iii.

5. Setting

6. Name some barriers that may prevent a change in behavior. may help you achieve success in behavior changes.

i.

ii.

iii.

7. Now give some ways to overcome the above barriers.

i.

ii.

iii.

8. Name some ways to help you manage time to improve your health.

i.

ii.

iii.

9. Reasonable weight loss is pounds per week.

10. If you eat more calories than you burn, you are in energy balance and you will weight.

11. If you burn more calories than you eat, you are in energy balance and you will weight.

12. Storing fat around the is associated with a higher risk for heart disease.

13. Name some benefits of physical activity for weight management.

i.

ii.

iii. 


\section{Study Guide Questions}

Session 5: Using what you've learned.

1. What are the keys to healthy eating?

2. What are some dietary planning principles?

3. What are some ways that you could save money when shopping?

4. How do grain products differ as far as nutrient quality?

5. T/F Eating low fat foods will definitely result in weight loss. Explain.

6. T/F Low fat foods always have much less calories that regular fat versions. Explain.

7. Name some low-fat flavoring alternatives.

8. Name some menu planning tips.

9. What are some tips you should keep in mind when dining out?

10. Name some benefits of physical activity.

11. Name some ways that you could incorporate physical activity into your daily life. 


\section{Study Guide Answers \\ Session 1: Introduction to Adolescent Health}

Section I: Introduction

B. Dietary Health Risks

1. Iron, Folate, Calcium, Vitamin A

2. Students who eat breakfast: Feel better in the morning, start the day ready to learn, are more alert and attentive, concentrate more in school,

Section II: Dietary Guidelines improve their test scores over those you skip breakfast.

1. The 3 principles that the dietary guidelines are based upon are Aim for fitness, Build a healthy base, and Choose sensibly.

Section III: Food Guide Pyramid

a. Bread, Cereals, rice, and pasta (6-11 servings)

1. Foods in the bread, cereals, rice, and pasta group provide many key nutrients, especially carbohydrate which is used for energy.

2. Nutrients found in this group include carbohydrate, B vitamins, iron, protein, and fiber.

3. To achieve 6-11 servings of this group: pack healthy grain snacks such as granola bars or cereal; eat a breakfast that includes cereal or whole grain toast.

b. Fruits (2-4 servings) and vegetables (3-5 servings)

1. Fruits and vegetables contain many nutrients. Some of these nutrients are vitamin A, vitamin C, folate, and iron.

2. Tips that you may use to increase your fruit and vegetable intake include: Snack on raw vegetables with a low-fat dip, add vegetables to pasta sauce for a delicious dish of primavera, carry a banana, apple or orange for a snack instead of a candy bar, freeze grapes or bananas for a frosty treat, try dried fruits like raisins and apricots as a snack, add fruits to low-fat vanilla yogurt, put in the blender, $\&$ whip up a smoothie.

c. Meat

1. Nutrients found in meat include protein, fat, B vitamins, and iron.

2. A serving of meat is $3 \mathrm{oz}$; about the size of a deck of cards.

3. A lot of the fat we consume comes from the meat group. Some ways to cut down on the fat intake from foods in this group include: Select lower-fat choices of beef and pork from the meat group (the fewer white specks in beef, the leaner it is.), select fish and poultry more often than beef, the most fat in poultry is in the skin - so remove it! cook in ways to reduce rather than add fat (broil, grill, or roast), cut off all visible fat from the meat before cooking and eating.

d. Milk, cheese, and yogurt

1. Milk and milk products are the best source of calcium in the diet. 
2. If you don't get enough calcium when you are young you are more prone to stress fractures and may increase your chances of getting osteoporosis when you are old.

3. Select low fat and fat free dairy products so you can get the required amounts of calcium without getting extra fat.

4. Teens need about $1300 \mathrm{mg}$ of calcium each day. A glass of skim milk contains $300 \mathrm{mg}$ of calcium, while a cup of low-fat yogurt contains 245 $\mathrm{mg}$. Regular fat dairy products often have less calcium than lower fat alternatives.

e. Fats, oils, and sweets

1. Some ways that you could lower the fat in your diet.

Choose lower-fat dairy products

Eat more poultry and fish than beef.

Trim the visible fat off meats.

Avoid eating the skin of animal products.

Use reduced fat dressings and toppings (sour cream).

Choose healthy snacks.

Section IV: Physical Activity

1. Some benefits of physical activity include:

Fun and relaxation

A healthy heart

Positive attitude

Strong bones and muscles

A healthy weight

Improved balance and coordination

Increased energy

Improved self-esteem

V. Types of physical activity include aerobic and strength and flexibility.

Aerobic increases your cardio-respiratory (heart and lungs) endurance, while strength exercise improves the work capacity of your skeletal muscles.

VI. Some ways that you could increase your daily physical activity are:

Walk your dog, ride your bike or walk to school or friend's house, park further away and walk rather than driving around for a close- up spot, do housework, garden or do yard work, dance to your favorite music, etc. 


\section{Study Guide Answers \\ Session 2: Overview of the Nutrients}

A. Carbohydrates

1. Carbohydrates should make up the majority of the calories of our diet.

2. Carbohydrates are your body's first choice for energy.

3. The food groups at the base of the pyramid contain good sources of carbohydrates. These are breads, pasta, and grains, fruits, and vegetables.

I. The 2 types of carbohydrates are simple and complex. Simple carbohydrates usually only contain calories and very little vitamins or minerals. Complex carbohydrates often contain nutrients such as B vitamins, folate, and iron. II. In general, complex carbohydrates are low in fat: however, many of the extras added to these carbohydrates (butter, cream sauces like alfredo, sour cream, dressings, mayonnaise, etc.) makes them fattening.

III. You could get more carbohydrates in your diet by:

Eating Breakfast. Choose convenient choices such as cereal, English muffins, bagels, and toast. Choose whole wheat!; Put dry cereal in a plastic bag and take it along for a quick snack or breakfast on the run. Eat it plain or mix with yogurt or skim milk if kept cold.; Toss low-fat cheese on a tortilla and pop it in the microwave for a few seconds. Roll it up and dip in salsa for a healthy, fast snack; Try low-fat granola-type cereals as a topper for fruit-flavored or frozen yogurt.

B. Protein

VII. Foods that are high in protein, but low in fat include; tuna and other fish, chicken and turkey without skin, lean meat with visible fat trimmed off, lowfat dairy products, soy (tofu), egg whites, and legumes (peas and beans)

VIII. Plant products have protein in them but often these proteins are "incomplete" and don't contain all of the essential amino acids. Therefore, a variety of plant foods should be combined, as each is limited in different amino acids. Combining more than one vegetable, such as beans and a tortilla forms a "complete" amino acid profile. Animal proteins, on the other hand, are "complete" and contain all of the essential amino acids.

IX. Some good sources of protein are: lean meat, eggs, fish, poultry (chicken and turkey), milk, soy, sweet potato, and legumes.

Peas and beans are legumes.

\section{Fat}

X. Some reasons why fat is an important part of the diet are: fats provide us with energy; although carbohydrate is the body's first choice for energy, fat makes up the body's reserves. Fat is a more dense form of energy offering 9 calories per gram compared to 4 calories in carbohydrates and protein. Fats also carry the fat-soluble vitamins (A, D, E, K). Without fat, the body cannot absorb these vitamins. Fat also provides insulation. Insulation helps maintain body temperature and also cushions internal organs from injury. 
XI. The unhealthy type of fat is saturated fat. It is considered unhealthy because it raises "bad cholesterol". Saturated fat is solid at room temperature. Some examples of foods that contain saturated fat are butter, egg yolks, whole milk, animal skin, creams (sour cream, cream cheese, whipping cream), bacon, pork, lard for cooking.

XII. The body needs fat for several functions in the body. The type of fat that is the healthiest is polyunsaturated. Some examples of foods that contain polyunsaturated fat are safflower oil, sunflower oil, cottonseed oil, and deepsea fish (salmon).

XIII. Some concerns with eating a high fat diet include excess calories resulting in overweight, heart disease from excess saturated fat and cholesterol, and increased risk of some cancers.

XIV. Some ways that you could easily lower the fat content of your diet are: Eat more vegetables, fruits, \& whole-grain products instead of cakes, cookies, \& French fries; Replace higher-fat foods with lower-fat, lower-calorie versions of the same food; Use low-fat toppings (salsa, reduced fat dressing) instead of high fat (sour cream, cheese spread); choose lower fat cooking methods broiled, grilled, steamed, or baked rather than fried or creamed.

1. Which is healthier: butter or margarine? From a calorie and fat standpoint, butter and margarine are similar, both with about 35 calories and 4 grams of fat per teaspoon. However, Butter being solid at room temperature and made from animal products contains saturated fat and cholesterol and is, therefore, unhealthier than margarine. Margarine, which contains mainly monounsaturated fat is made from vegetable oil and contains no cholesterol.

\section{Vitamins}

1. Vitamins assist in many of the metabolic processes in the body such as converting food to energy or protecting cells from destruction.

2. There are categories of vitamins: fat-soluble $(\mathrm{A}, \mathrm{D}, \mathrm{E}, \mathrm{K})$ and water-soluble (Bs and C). Fat-soluble vitamins are stored in the body, water-soluble are generally not stored in large amounts. Fat-soluble are excreted through the feces, while water-soluble are excreted through the urine.

3. Name a food that is a good source of the following vitamin:

\begin{tabular}{|l|l|l|}
\hline Vitamin & Function & Food Source \\
\hline Vitamin A & Vision & $\begin{array}{l}\text { Lean meat, dark orange/yellow } \\
\text { vegetables }\end{array}$ \\
\hline $\begin{array}{l}\text { B } \\
\text { vitamins }\end{array}$ & $\begin{array}{l}\text { Conversion of food to } \\
\text { energy }\end{array}$ & $\begin{array}{l}\text { Breads, grains, pasta, fruits and } \\
\text { vegetables }\end{array}$ \\
\hline Vitamin C & Antioxidant & Citrus fruits \\
\hline Vitamin D & Builds bones & Low-fat dairy products \\
\hline Vitamin E & Antioxidant & Vegetables, plant oils \\
\hline Vitamin K & Helps blood clot & Green leafy vegetables \\
\hline
\end{tabular}


4. You get all of your vitamins without taking a vitamin supplement by eating a well-balanced diet.

E. Minerals

1. Minerals are involved in many important processes in your body.

2. Three major minerals that are important in your body are iron, calcium, and sodium.

3. What is the function of the above listed minerals?

\begin{tabular}{|l|l|}
\hline Mineral & Function \\
\hline Iron & Builds healthy red blood cells \\
\hline Calcium & Builds strong bones \\
\hline Sodium & $\begin{array}{l}\text { Important in water balance and } \\
\text { blood pressure regulation. }\end{array}$ \\
\hline
\end{tabular}

4. You do to be sure that you get the kinds and amounts of minerals that you need each day by eating a well-balanced diet.

\section{F. Water}

1. Lack of fluid results in the quickest loss of physical performance. Some functions of water include: Transport - blood, hormones, wastes; All chemical reactions in the body take place in water; Protection of tissues and spinal cord; Proper sensory function; Regulation of body temperature; Digestion and absorption

2. Tips that would be reasonable to increase your fluid intake each day include: bring a water bottle with you, drink more fruit juices, drink on schedule rather than waiting until you are thirsty.

3. You can assess your own hydration status by looking at the color of your urine. A light yellow would indicate diluted urine illustrating proper hydration, while a darker yellow indicates a concentrated urine, or poor hydration status. 


\section{Study Guide Answers}

Obesity

\section{Session 3: Nutrition for Life}

1. Overweight and obesity are the most prevalent nutritional diseases of children and adolescents in the US.

2. Being overweight in adolescence can affect your ability to maintain a healthy weight as an adult. This is because in adolescence, your fat cells increase in size as well as number. As a result, an overweight teen may develop more fat cells and therefore have a more difficult time losing weight as an adult.

Healthy Weight

3. BMI is body mass index. It is the ratio of height to weight and can be used to estimate health status.

4. Measurements of BMI comes from height and weight

5. The normal range for $\mathrm{BMI}$ is $20-24.9$.

Underweight $<18.5$ Overweight $25-29.9$ Obese $30-39.9$

Answers will vary.

6. BMIs that are too high or too low can affect health status. Being underweight makes a person more susceptible to infection, while being overweight makes a person more susceptible to heart disease, diabetes, and some cancers.

7. BMI values be carefully interpreted because they don't account for large amounts of muscle mass. A very athletic person with a lot of muscle mass may appear to have an unhealthy BMI although they have a small amount of body fat.

\section{Type 2 Diabetes}

8. Insulin signals the cells to let glucose inside.

9. Overweight and obesity affects insulin function by altering the cell shape and making the cell unable to recognize the insulin. As a result, glucose stays in the blood and does not enter the cells. This leads to type 2 diabetes and can have many adverse complications

10. The most common type of diabetes is type 2 diabetes.

11. The age group 12-19 is at the highest risk

12. Some of the complications of type 2 diabetes are heart disease, vision loss, kidney disease, skin disorders, foot problems, gum disease.

Heart Disease

13. Heart disease is the leading cause of death among Americans.

14. Some risk factors associated with heart disease are high fat, especially saturated fat diets, diets low in fruits and vegetables, being overweight or obese, development of type 2 diabetes.

15. You lower your risks of heart disease by: Eating a heart-healthy diet! Practice balance, variety and moderation using the Food Guide Pyramid. Choose foods that are low in total fat, saturated fat and cholesterol; Exercise - Every day!; Maintain your weight! Always strive to maintain a healthy body weight; Eat more fruits, vegetables and whole-grain breads and cereals; don't smoke! 
Session 4: Taking Responsibility for Your Health

1. Some health problems associated with poor diet and physical inactivity are overweight and obesity, type 2 diabetes, heart disease, and some cancers.

2 . It is easier to make dietary changes when you have support from your friends and family!

3. Self-efficacy or confidence is defined as believing that you have the ability to make behavior changes.

4. Some things that can affect your ability to make behavior changes are Awareness- the effects of changing your behavior, Barriers - things that could stop you from making these changes, Attitude- readiness to make a change, Support - from your family and friends.

5. Setting goals may help you achieve success in behavior changes.

6. Some barriers that may prevent a change in behavior are lack of time or money, lack of support from family and friends, and lack of availability of resources

7. Taking responsibility for your actions, making good decisions regarding your health, along with a sincere dedication to succeed can make overcoming these barriers a little easier. Setting realistic goals, good time management, and social support from your family and friends may help alleviate some of the above listed barriers.

8. Time management involves planning ahead for healthier choices. For example, pack a lunch, bring healthy snacks with you, don't skip meals- this will help prevent overeating later in the day, set aside a time slot for physical activity, join a walking/exercise club or form one of your own

9. Reasonable weight loss is 1-2 pounds per week.

10. If you eat more calories than you burn, you are in positive energy balance and you will gain weight.

11. If you burn more calories than you eat, you are in negative energy balance and you will lose weight.

12. Storing fat around the abdomen is associated with a higher risk for heart disease.

13. Some benefits of physical activity for weight management are: increased energy expenditure, increased metabolism, controls appetite, psychological benefit, and helps maintain muscle mass. 


\section{Study Guide Answers}

Session 5: Using what you've learned.

1. The keys to healthy eating are balance, variety, and moderation:

Eat a balanced meal with a variety of foods, but remember to eat in moderation (not too much fat and sugar!).

2. Some dietary planning principles are: Adequacy - sufficient energy and nutrients, Balance - enough, but not too much of each type of food, Nutrient Density - the most nutrients for least calories, Variety - all food groups \& vary choices.

3. Some ways that you could save money when shopping are: Planning is the key to saving money, make a shopping list, clip any coupons for foods or items that you would normally use.

4. Grain products differ as far as nutrient quality depending on the processing: Refined - often lose nutrients in processing, Enriched - have lost nutrients but they have been added back, Whole grain - are usually rich in all nutrients

5. False. Fat matters but calories count. A calorie is a calorie whether it is from protein, fat, or carbohydrate, you can lose weight by eating less calories and expending more calories. However, a healthier diet includes one that is low in fat.

6. False. Low fat foods don't always have fewer calories than regular fat versions.

Although they may be healthier as far as disease, they may not afford a benefit in weight loss.

7. Some low-fat flavoring alternatives are:

Herbs - oregano, basil, cilantro, parsley; Spices - cinnamon, nutmeg, pepper, paprika; Reduced fat dressing; Mustard; Catsup; Fat-free mayonnaise; Fat-free or reduced fat sour cream; Salsa; Jelly or Jam.

8. To better assist you in planning a healthy menu:

Read the Nutrition Facts labels on food products. You can find out how many calories, fat grams, milligrams of sodium and amounts of other nutrients are in the food you are about to eat. Plan your meals for the week. Make a shopping list and buy only what you need. This will save both time and money. Plan meals with foods that vary in shape, color, texture, temperature and flavor.

9. When dining out ask for what you want - substitute cooking methods when possible. Choose lower fat cooking methods (baked instead of fried, broiled instead of creamed)

10. Some benefits of physical activity include: Increases energy expenditure aids in weight loss; reduces risk of heart disease; relieves stress; decreases illnesses; increases energy; helps maintain bone health; decreases risk of diabetes, Builds self-esteem.

11. Some ways that you could incorporate physical activity into your daily life are: Begin slowly; Do activities that you enjoy; Set realistic goals; Reward yourself for reaching your goals; (With a movie, $\mathrm{CD}$, etc. - not with food); Gradually increase time and intensity. 


\title{
Appendix C
}

\author{
Data Collection Tools
}

24-Hour Dietary Recall Form

Food Frequency Questionnaire

Weight and Height Record

Nutrition Knowledge Questionnaire

Physical Activity Questionnaire

Dietary and Exercise Confidence Survey

Social Support Survey 


$$
\text { 2'1-Hour Recall }
$$

Day of the Week

Date

\section{Breakfast}

\begin{tabular}{|l|l|}
\hline Type or Brand of Food & Amount \\
\hline & \\
\hline & \\
\hline & \\
\hline & \\
\hline & \\
\hline & \\
\hline
\end{tabular}

\section{Mid-Morning Snacks and Beverages}

\begin{tabular}{|l|l}
\hline Type or Brand of Food & Amount \\
\hline
\end{tabular}

Lunch

Type or Brand of Food Amount

\begin{tabular}{|l|l|}
\hline Afternoon Snack & \\
\hline Type or Brand of Food & Amount \\
\hline & \\
\hline & \\
\hline & \\
\hline & \\
\hline & \\
\hline
\end{tabular}

\begin{tabular}{|l|l|}
\hline Dinner & \\
\hline Type or Brand of Food & Amount \\
\hline & \\
\hline & \\
\hline & \\
\hline & \\
\hline & \\
\hline & \\
\hline & \\
\hline
\end{tabular}

Evening Snacks and Beverages

\begin{tabular}{|l|l|}
\hline Type or Brand of Food & Amount \\
\hline & \\
\hline & \\
\hline
\end{tabular}


ID\#

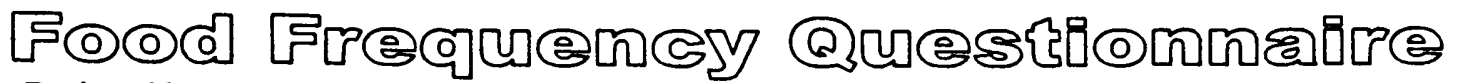

Designed by $\mathbf{M}$. Buzzard et al.

Directions: Please answer these questions as honestly as possible. There are no right or wrong answers. Think about what you usually eat when you answer these questions.

Check only one answer for each question.

1. How often do you eat white bread or rolls? (Include bread for sandwiches or toast.)

$\square$ never

$\square$ less than once a WEEK

ㄱ 1-3 times a week

[ $4-6$ times a week

$\square$ Once a day

2 times a day

$\square 3$ or more times a DAY

2. How often do you eat whole wheat bread, rye bread, or other brown bread or rolls?

never

less than once a WEEK

$\square \quad$ 1-3 times a week

$\square$ 4-6 times a week

$\square$ Once a day

- 2 times a day

$\square 3$ or more times a DAY

3. How often do you eat cold or hot cereal or grits?

never

$\square$ less than once a WEEK

1-3 times a week

$\square$ 4-6 times a week

$\square$ Once a day

- 2 times a day

ㄴ 3 or more times a DAY

4. How often do you eat pizza?

never

less than once a WEEK

- 1-3 times a week

$\square$ 4-6 times a week

$\square$ Once a day

$\square \quad 2$ times a day

$\square \quad 3$ or more times a DAY 
5. How often do you drink milk or add milk to your cereal?

$\checkmark$ never

$\square$ less than once a WEEK

ㄱ 1-3 times a week

ㄱ 4-6 times a week

$\square$ Once a day

$\square \quad 2$ times a day

․ 3 or more times a DAY

6. How often do you eat cheese? (Include cheese in sandwiches or cheeseburgers.)

$\square$ never

$\square$ less than once a WEEK

ㅁ 1-3 times a week

$\square$ 4-6 times a week

$\square$ Once a day

$\square 2$ times a day

$\square 3$ or more times a DAY

7. How often do you drink fruit or vegetable juice?

$\square$ never

$\square$ less than once a WEEK

ㅁ 1-3 times a week

$\square$ 4-6 times a week

$\square$ Once a day

$\square 2$ times a day

$\square 3$ or more times a DAY

8. How often do you eat fruit? (Include all kinds - fresh, canned, cooked, or dried.)

never

$\square$ less than once a WEEK

ㅁ 1-3 times a week

ㄴ 4-6 times a week

$\square$ Once a day

ㄴ 2 times a day

ㄱ 3 or more times a DAY

9. How often do you eat beans like baked beans, red beans, black beans, or blackeyed peas? (Include beans in chili, bean soup, and beans and rice).

never

$\square$ less than once a WEEK

ㄱ 1-3 times a week

$\square$ 4-6 times a week

$\square$ Once a day

a times a day

口 3 or more times a DAY 
10. How often do you eat green salads, like lettuce or spinach salad?

$\square$ never

$\square$ less than once a WEEK

J 1-3 times a week

$\sqsupset$ 4-6 times a week

$\sqsupset$ Once a day

כ 2 times a day

ㄱ 3 or more times a DAY

11. How often do you eat french fries, fried potatoes, or hash browns?

never

less than once a WEEK

1-3 times a week

4-6 times a week

$\square$ Once a day

2 times a day

ㄱ 3 or more times a DAY

12. How often do you eat baked, boiled, or mashed potatoes?

never

$\square$ less than once a WEEK

ㄱ 1-3 times a week

ㄱ 4-6 times a week

$\square$ Once a day

2 times a day

ㄱ 3 or more times a DAY

13. How often do you eat vegetables? (Include all kinds - fresh, canned, cooked.)

$\square$ never

$\square$ less than once a WEEK

- 1-3 times a week

ㄱ 4-6 times a week

$\square$ Once a day

ㄱ 2 times a day

ㅁ 3 or more times a DAY

14. How often do you eat fried chicken or chicken nuggets?

never

$\square$ less than once a WEEK

ㅁ 1-3 times a week

ㄴ 4-6 times a week

$\square$ Once a day

J 2 times a day

ㄱ 3 or more times a DAY 
15. How often do you eat fried fish?

$\sqsupset$ never

$\square$ less than once a WEEK

- 1-3 times a week

- 4-6 times a week

$\square$ Once a day

J 2 times a day

口 3 or more times a DAY

16. How often do you eat hot dogs?

$\square$ never

$\square$ less than once a WEEK

- 1-3 times a week

ㄴ 4-6 times a week

$\square$ Once a day

$\square \quad 2$ times a day

口 3 or more times a DAY

17. How often do you eat bologna, salami, or other lunchmeat?

never

$\square$ less than once a WEEK

1-3 times a week

4-6 times a week

Once a day

$\square \quad 2$ times a day

ㄱ or more times a DAY

18. How often do you eat mayonnaise on your sandwiches or burgers?

$$
\text { never }
$$

less than once a WEEK

- 1-3 times a week

- 4-6 times a week

Once a day

․ 2 times a day

ㄱ 3 or more times a DAY

19. How often do you eat bacon or sausage?

never

less than once a WEEK

1-3 times a week

4-6 times a week

Once a day

$\square 2$ times a day

ㄱ 3 or more times a DAY 
20. How often do you eat hamburgers, cheeseburgers, meatloaf, or meatballs?

$\square$ never

$\square$ less than once a WEEK

ㄴ 1-3 times a week

[] 4-6 times a week

Once a day

$\square 2$ times a day

3 or more times a DAY

21. How often do you eat chips? (Include potato chips, corn chips, tortilla chips, and cheetos.)

never

less than once a WEEK

1-3 times a week

$\square$ 4-6 times a week

Once a day

$\square 2$ times a day

ㄱ 3 or more times a DAY

22. How often do you eat donuts, sweet rolls (Honey buns), or muffins?
never
less than once a WEEK
1-3 times a week
- 4-6 times a week
$\square$ Once a day
2 times a day
$\square 3$ or more times a DAY

23. How often do you eat cake, cookies, brownies, or pie?

never

$\square$ less than once a WEEK

- 1-3 times a week

4-6 times a week

Once a day

$\square 2$ times a day

3 or more times a DAY

24. How often do you eat ice cream or milkshakes?

never

less than once a WEEK

1-3 times a week

4-6 times a week

$\square$ Once a day

$\square 2$ times a day

- 3 or more times a DAY 
25 . How often do you eat candy bars?

$\square$ never

$\square$ less than once a WEEK

ㄷ 1-3 times a week

ㄷ 4-6 times a week

$\square$ Once a day

- 2 times a day

$\square 3$ or more times a DAY

26. How often do you add butter or margarine to your bread or rolls

$\square$ Never

ㄴot very often

$\square$ Sometimes

․ Almost always

$\square$ Don't eat bread or rolls

27. Please list the names of one or two cereals you eat most often:

1.

2.

$\square$ Check here if you don't eat cereal

28. What kind of milk do you usually drink?

$\square$ whole milk

$\square$ low-fat milk (1or 2\%)

$\square$ skim (non-fat) milk

$\square$ don't know

$\square$ don't drink milk

29 . What type of juice do you usually drink?

$\square 100 \%$ real fruit or vegetable juice

$\square$ flavored fruit drinks (such as Hi-C, Kool-Aid, Sunny D, or punch)

$\square$ don't know

$\square$ don't drink juice

30. How often do you add butter, margarine, sour cream or gravy to your potatoes?

$\square$ Never

$\square$ Not very often

口 Sometimes

․ Almost always

$\square$ Don't eat potatoes

31. How often do you add butter or margarine to your vegetables?

$\square$ Never

- Not very often

$\square$ Sometimes

- Almost always

¿ Don't eat vegetables 
32. How often do you take the skin off before eating fried chicken?

$\square$ Never

Not very often

$\square$ Sometimes

$\square$ Almost always

$\square$ Don't eat fried chicken

33. When there is fat on your meat, do you:

$\checkmark$ Usually eat the fat

$\square$ Sometimes eat the fat

Usually cut the fat off

$\square$ Don't eat meat with fat on it

$\square$ Don't eat meat

34. How often do you add salad dressing to your lettuce or green salad?

$\square$ Never

$\square$ Not very often

$\square$ Sometimes

- Almost always

$\square$ Don't eat salads

35. Please check each of the following kinds of low-fat foods you usually eat: low-fat cheese

_ low-fat mayonnaise low-fat salad dressing low-fat chips low-fat ice cream low-fat cakes or cookies don't eat low-fat or fat-free foods

36. How often do you read nutrition labels on the foods you eat?

$\square$ Never

Almost never

ㄷ Sometimes

Fairly often

V Very often

Thank You for completing the questionnaire. Feel free to add any additional comments below. 
ID\#

\section{Height and Weight Record Form}

Date

Gender

Weight in Pounds

Height in Inches

Calculated Body Mass Index 
ID \#

Parmenter and Wardle's (1998) Nutrition Knowledge Questionnaire

The first few items are about what advice you think experts are giving us.

1. Do you think health experts recommend that people should be eating more, the same amount, or less of these foods. (Check $(\sqrt{ })$ one box for each food)

Vegetables

Sugary foods

Meat

Starchy foods

High fiber foods

Fruit

Salty foods

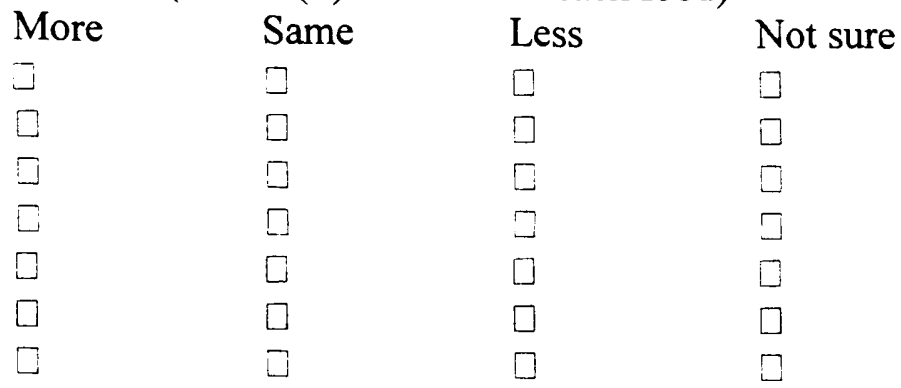

2. How many servings of fruits and vegetables a day do you think experts are advising people to eat? (One serving could be 1 apple or a handful of chopped carrots)
a) $1-2$ servings
b) 2-4 servings
c) 3-5 servings
d) 5 or more servings

3. Which fat do experts say it is most important to cut down on? (Check $(\sqrt{ })$ one box)
a) Monounsaturated
b) Polyunsaturated
c) Saturated
d) None

4. What version of dairy products do experts say people should eat? (Check $(\sqrt{ })$ one box)
a) Full fat
b) Lower fat
c) Mixture of full fat and low fat
d) Neither, dairy foods should be cut out

Experts classify foods into groups. We are interested to see whether people are aware what foods are in these groups.

1. Do you think these foods are high or low in sugar? (Check $(\sqrt{ })$ one box for each food)

Bananas High

Plain Yogurt

Ice Cream

Squash

Ketchup

Canned fruit

$\begin{array}{lll}\text { High } & \text { Low } & \text { No } \\ \square & \square & \square \\ \square & \square & \square \\ \square & \square & \square \\ \square & \square & \square \\ \square & \square & \square \\ \square & \square & \square\end{array}$


2. Do you think these foods are high or low in fat? (Check $(\sqrt{ })$ one box for each food)

Pasta (without sauce)

Margarine

Baked beans

Lunchmeat

Honey

Eggs

Nuts

Bread

Cottage Cheese

Butter

High Low Not sure

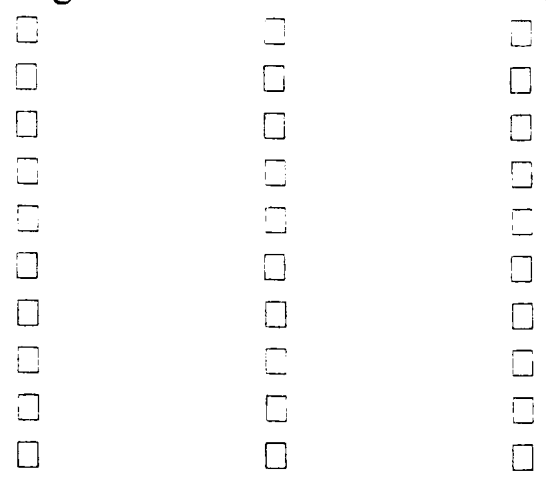

3. Do you think experts put these in the complex carbohydrates (starchy foods) group? (Check $(\sqrt{ })$ one box for each food)

$\begin{array}{llll}\text { Cheese } & \text { Yes } & \text { No } & \text { Not } \\ \text { Pasta } & \square & \square & \square \\ \text { Butter } & \square & \square & \square \\ \text { Rice } & \square & \square & \square \\ \text { Oatmeal } & \square & \square & \square \\ & \square & \square & \square\end{array}$

4. Do you think these foods are high or low in salt? (Check $(\sqrt{ })$ one box for each food) High Low

Not sure

Sausage

Pasta

Red meat

Frozen vegetables

Canned vegetables

Cheese

5. Do you think these foods are high or low in protein? (Check $(\sqrt{ })$ one box for each food)

Chicken

Cheese

Fruit

Baked beans

Butter

Milk

High Low Not sure


6. Do you think these foods are high or low in fiber (roughage)? (Check $(\sqrt{ })$ one box for each food)

\begin{tabular}{|c|c|c|c|}
\hline & High & Low & Not sure \\
\hline Cornflakes & {[} & z & $\Xi$ \\
\hline Bananas & $\Xi$ & $=$ & C \\
\hline Eggs & $\Xi$ & $\Xi$ & $=$ \\
\hline Red meat & $\bar{z}$ & $\Xi$ & $\Xi$ \\
\hline Broccoli & こ & $\Xi$ & 3 \\
\hline Nuts & כ & $\square$ & 0 \\
\hline Fish & Ј & E & $=$ \\
\hline Baked potato \& skin & E & $E$ & $\Xi$ \\
\hline Chicken & ב & {[} & $\square$ \\
\hline Baked beans & $\square$ & $\square$ & $\square$ \\
\hline
\end{tabular}

7. Do you think these fatty foods are high or low in saturated fat? (Check $(\sqrt{ })$ one box for each food)

Tuna

Whole milk

Olive oil

Red meat

Margarine

Chocolate

8. Some foods contain a lot of fat but not a lot of cholesterol. (Check $(\sqrt{ })$ one box)
a. Agree
b. Disagree
c. Not sure

$\begin{array}{lll}\text { High } & \text { Low } & \text { Not } \\ \square & \square & \square \\ \square & \square & \Xi \\ \square & \square & \square \\ \square & \square & \square \\ \square & \square & \square \\ \square & \square & \square\end{array}$

9. Do you think experts call these a healthy alternative to red meat? (Check $(\sqrt{ })$ one box for each food)

$\begin{array}{llll} & \text { Yes } & \text { No } & \text { Not } \\ \text { Soy Burger } & \square & \square & \square \\ \text { Lunchmeat } & \square & \square & \square \\ \text { Baked beans } & \square & \square & \square \\ \text { Nuts } & \square & \square & \square \\ \text { Low fat cheese } & \square & \square & \square \\ \text { Omelet } & \square & \square & \square\end{array}$

10. A glass of unsweetened fruit juice counts as a serving of fruit. (Check $(\sqrt{ })$ one box)
a. Agree
b. Disagree
c. Not sure

Not sure 
11. Saturated fats are mainly found in (Check $(\sqrt{ })$ one box)
a. Vegetable oils
b. Dairy products
c. Both $a$ and $b$
d. Not sure

12. Brown sugar is a healthy alternative to white sugar. (Check $(\sqrt{ })$ one box)
a. Agree
c. Disagree
c. Not sure

13. There is more protein in a glass of whole milk than in a glass of skim milk. (Check $(\sqrt{ })$ one box)
a. Agree
b. Disagree
c. Not sure

14. Margarine contains less fat than butter. (Check $(\checkmark)$ one box)
a. Agree
b. Disagree
c. Not sure

15. Which of these breads contains the most vitamins and minerals? (Check $(\sqrt{ })$ one box)
a. White
b. Brown
c. Whole grain
d. Not sure

16. Which do you think is higher in calories butter or margarine? (Check $(\sqrt{ })$ one box)
a. Butter
b. Margarine
c. Both the same
d. Not sure

17. A type of oil that contains mostly monounsaturated fat is (Check $(\sqrt{ })$ one box)
a. Coconut oil
b. Sunflower oil
c. Olive oil
d. Palm oil
e. Not sure

18. There is more calcium in a glass of whole milk than in a glass of skim milk. (Check $(\sqrt{ })$ one box)
a. Agree
b. Disagree
c. Not sure 
19. Which of the following has the most calories for the same weight? (Check $(\sqrt{ })$ one box)
a. Sugar
b. Starchy foods
c. Fiber/roughage
d. Fat
e. Not sure

20. Harder fats (fats that are more solid at room temperature) contain more fat? (Check $(\sqrt{ })$ one box)
a. Monounsaturated $\square$
b. Polyunsaturated
c. Saturated
d. Not sure

21. Polyunsaturated fats are mainly found in: (Check $(\sqrt{ })$ one box $)$
a. Vegetable oils
b. Dairy products
c. Both $\mathrm{a}$ and $\mathrm{b}$
d. Not sure

The next few items are about choosing foods. Please answer what is being asked and not whether you like or dislike the food. For example, suppose you were asked...

"If a person wanted to cut down of fat, which cheese would be best to eat

a) Cheddar cheese

b) Blue cheese

c) Cream cheese

d) Cottage cheese

If you didn't like cottage cheese, but knew it was the right answer, you would still check cottage cheese.

1. Which would be the best choice for a low-fat, high fiber snack? (Check $(\sqrt{ })$ one box)
a. Sugar-free strawberry yogurt
b. Raisins or other dried fruit
c. Cereal bar
d. Whole wheat crackers and cheese

2. Which would be the best choice for a low-fat, high fiber meal? (Check $(\sqrt{ })$ one box)
a. Grilled chicken
b. Cheese on whole wheat toast
c. Beans and brown rice
d. Tuna casserole 
3. Which kind of sandwich do you think is healthier? (Check $(\sqrt{ })$ one box)
a. Two thick slices of bread with a thin slice of cheese
b. Two thin slices of bread with a thick slice of cheese

4. Many people eat spaghetti and meat sauce. Which do you think is healthier? (Check ( $\sqrt{ })$ one box)
a. Large amount of pasta with a little meat sauce
b. Small amount of pasta with a lot of meat sauce

5. If a person wanted to reduce the amount of fat in their diet, which one would be the best choice? (Check $(\sqrt{ })$ one box $)$
a. Steak, grilled
b. Sausages, grilled
c. Turkey breast, grilled
d. Pork chop, grilled

6. If a person wanted to reduce the amount of fat in their diet, but didn't want to give up French fries, which one would be the best choice? (Check $(\sqrt{ })$ one box)
a. Thick cut
b. Thin cut, crispy
c. Crinkle cut

7. If a person wanted something sweet, but was trying to cut down on sugar, which one would be the best choice? (Check $(\sqrt{ })$ one box)
a. Honey on toast
b. A cereal snack bar
c. Plain donut (unglazed)
d. Banana with plain yogurt

8. Which one of these would be the healthiest dessert? (Check $(\sqrt{ })$ one box)
a. Baked cinnamon apple
b. Strawberry yogurt
c. Whole wheat crackers with cheddar cheese
d. Carrot cake with cream cheese topping

9. Which cheese would be the lowest fat option? (Check $(\sqrt{ })$ one box)
a. Plain cream cheese
b. Swiss
c. Cheddar
d. Blue Cheese 
10. If a person wanted to reduce the salt in their diet, which one would be the best choice? (Check $(\sqrt{ })$ one box)
a. Ready made frozen dinner
b. Ham with pineapple
c. Vegetable omelet
d. Stir fry vegetables with soy sauce

This section is about health problems or diseases.

1. Are you aware of any major health problems or diseases that are related to a low intake of fruits and vegetables? (Check $(\sqrt{ })$ one box)
a. Yes
b. No
c. Not sure

If yes, what health problems do you think are related to low intake of fruit and vegetables?

2. Are you aware of any major health problems or diseases that are related to a low intake of fiber? (Check $(\sqrt{ })$ one box)
a. Yes
b. No
c. Not sure

If yes, what health problems do you think are related to low intake of fiber?

3. Are you aware of any major health problems or diseases that are related to how much sugar people eat? (Check $(\sqrt{ })$ one box)
a. Yes
b. No
c. Not sure

If yes, what health problems do you think are related sugar?

4. Are you aware of any major health problems or diseases that are related to how much salt or sodium people eat? (Check $(\sqrt{ })$ one box)
a. Yes
b. No
c. Not sure

If yes, what health problems do you think are related salt? 
5. Are you aware of any major health problems or diseases that are related to how much fat people eat? (Check $(\sqrt{ })$ one box)
a. Yes
b. No
c. Not sure

If yes, what health problems do you think are related to too much fat?

6. Do you think these help reduce the chances of getting certain kinds of cancer? (Check $(\sqrt{ })$ one box for each suggestion)

$\begin{array}{llll}\text { Eating more fiber } & \text { Yes } & \text { No } & \text { Not sure } \\ \text { Eating less sugar } & \square & \square & \square \\ \text { Eating less fruit } & \square & \square & \square \\ \text { Eating less salt } & \square & \square & \square \\ \text { Eating more fruit and vegetables } & \square & \square & \square \\ \text { Eating less preservatives/additives } & \square & \square & \square\end{array}$

7. Do you think these help prevent heart disease? (Check $(\sqrt{ })$ one box for each suggestion).

Eating more fiber

Eating less saturated fat

Eating less salt

Eating more fruit and vegetables

Eating less preservatives/additives

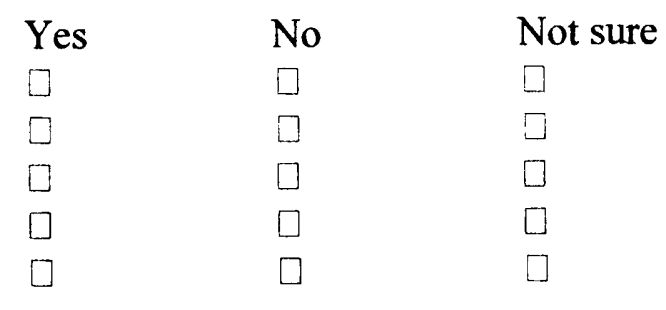

8. Which one of these is more likely to raise one's cholesterol level? ? (Check $(\sqrt{ })$ one box)
a. Antioxidants
b. Polyunsaturated fat
c. Saturated fat
d. Cholesterol in the diet
e. Not sure

9. Which of these are antioxidant vitamins? (Check $(\sqrt{ })$ yes for all that are and no for all that are not)
a. Vitamin A
b. B vitamins
c. Vitamin C
d. Vitamin D
e. Vitamin E
f. Vitamin $\mathrm{K}$

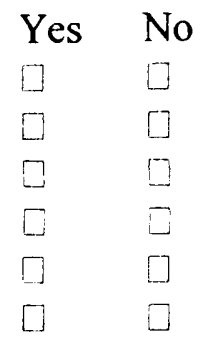


1. Are you male or female? ? (Check $(\sqrt{ })$ one box)
a. Male
b. Female

2. How old are you? ? (Check $(\sqrt{ })$ one box)
a. 13 or younger
b. 14
c. 15
d. 16
e. 17
f. 18 or older

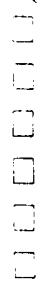

3. What is your ethnic origin? ? (Check $(\sqrt{ })$ one box)
a. White
b. Black American
c. Black Caribbean
d. Black other
e. Hispanic
Please specify
f. Indian/Middle Eastern
g. Asian
Please specify
h. Mixed ethnicity
Please specify
i. Any other not listed
Please specify

4. What grade are you in? ? (Check $(\sqrt{ })$ one box)
a. $9^{\text {th }}$
b. $10^{\text {th }}$
b. $11^{\text {th }}$
c. $12^{\text {th }}$

5. What is the highest level of education completed by your parents? ? (Check $(\sqrt{ })$ one box for each parent)
a. Didn't complete HS or equivalent
b. GED/ Technical school
c. Completed HS
d. Some college
e. Graduated college (Bachelor's)
f. Graduate degree (Master's, $\mathrm{PhD}$ )
g. Other
Please specify 
6. Do you currently have a job? ? (Check $(\sqrt{ })$ one box)
a. Yes
[]
b. No
$\square$

7. Are you dieting? (Check $(\sqrt{ })$ one box)
a. Yes
b. No

If yes, what type of diet are you on?

\section{THE END}

Thank you very much for your time. If there are any comments that you would like to make, please do so below. They would be very welcome. 
ID\#

\section{Kowalski’s Physical Activity Questionnaire (High School)}

Age:

Race:

Sex: $M$

$\mathrm{F}$

Grade:

We are trying to find out about your level of physical activity from the last $\overline{7 \text { days (in the }}$ last week). This includes sports or dance that make you sweat or make your legs feel tired, or games that make you breathe hard, like tag, skipping, running, climbing, and others.

Remember:

There are no right and wrong answers - this is not a test.

Please answer all the questions as honestly and accurately as you can - this is very important.

1. Physical activity in your spare time: Have you done any of the following activities in the past 7 days (last week)? If yes, how many times? (Mark only one circle per row.)

\section{None $\quad 1-2 \quad 3-4 \quad 5-6 \quad$ or more}

Weight Lifting

Rowing/canoeing

In-line skating

Basketball

Walking for exercise

Bicycling

Jogging or running

Aerobics

Swimming

Baseball, softball

Dance

Football

Racquetball/Handball

Skateboarding

Soccer

Street hockey

Volleyball

Floor hockey

Ice skating

Ice hockey

Other: 
2. In the last 7 days, during your physical education (PE) classes, how often were you very active (playing hard, running, jumping, throwing)? (Check one only.)

I don't do PE

Hardly ever

Sometimes

Quite often

Always

3. In the last 7 days, what did you normally do at lunch (besides eating lunch)? (Check one only.)

Sat down (talking, reading, doing schoolwork)

Stood around or walked around

Ran or played a little bit

Ran around and played quite a bit

Ran and played hard most of the time

4. In the last 7 days, on how many days right after school, did you do sports, dance, or play games in which you were very active? (Check one only.)

None

1 time last week

2 or 3 times last week

4 times last week

5 times last week

5. In the last 7 days, on how many evenings did you do sports, dance, or play games in which you were very active? (Check one only.)

None

1 time last week

2 or 3 times last week

4 or 5 last week

6 or 7 times last week

6. On the last weekend, how many times did you do sports, dance, or play games in which you were very active? (Check one only.)

None

1 time

$2-3$ times

$4-5$ times

6 or more times 
7. Which one of the following describes you best for the last 7 days? Read all five statements before deciding on the one answer that describes you.

All or most of my free time was spent doing things that involve little physical effort

I sometimes ( $1-2$ times last week) did physical things in my free time

(e.g. played sports, went running, swimming, bike riding, did aerobics)

I often ( $3-4$ times last week) did physical things in my free time

I quite often ( $5-6$ times last week) did physical things in my free time ......

I very often ( 7 or more times last week) did physical things in my free time ...

8. Mark how often you did physical activity (like playing sports, games, doing dance, or any other physical activity) for each day last week.
Little
None bit Medium Often often

Monday

Tuesday

Wednesday

Thursday

Friday

Saturday

Sunday

9. Were you sick last week, or did anything prevent you from doing your normal physical activities? (Check one.)

Yes

No

If Yes, what prevented you? 
Below is a list of things people might do while trying to change their eating habits. We are mainly interested in what you eat, rather than weight reduction.

Whether you are trying to change your eating habits or not, please rate how confident you are that you could really motivate yourself to do things like these consistently, for at least six months.

Please circle one number for each item:

How sure are you that you can do these things?

\begin{tabular}{ccccc}
$\begin{array}{c}\text { I know I } \\
\text { cannot } \\
1\end{array}$ & 2 & $\begin{array}{c}\text { Maybe I } \\
\text { can }\end{array}$ & 4 & $\begin{array}{c}\text { I know I } \\
\text { can }\end{array}$ \\
\hline
\end{tabular}

1. Stick to your low fat, low salt foods when you feel depressed, bored, or tense. $\quad \begin{array}{lllllll}1 & 2 & 3 & 4 & 5\end{array}$

2. Stick to your low fat, low salt foods when there is high fat, high salt food readily available at a party.

3. Stick to your low fat, low salt foods when dining with friends or co-workers.

23

4. Stick to your low fat, low salt foods when the only snack close by is available from a vending machine.

5. Stick to your low fat, low salt foods when you are alone, and there is no one to watch you.

6. Eat smaller portions so there are no leftovers.

2

2

7. Cook smaller portions so there are no leftovers.

23

8. Eat lunch as your main meal of the day, rather than dinner.

9. Eat smaller portions of food at a party. 
10. Eat salads for lunch.

$\begin{array}{lllll}1 & 2 & 3 & 4 & 5\end{array}$

11. Add less salt than the recipe calls for.

$1 \quad 2 \quad 3$

12. Eat unsalted, peanuts, chips, crackers, and pretzels.

$\begin{array}{lllll}1 & 2 & 3 & 4 & 5\end{array}$

13. Avoid adding salt at the table. 1

$\begin{array}{llll}2 & 3 & 4 & 5\end{array}$

14. Eat unsalted, unbuttered popcorn.

1

23

4

5

15. Keep the salt shaker off the kitchen table.

1

16. Eat meatless (vegetarian) entrees for dinner. 1

3

4

5

17. Substitute low or non-fat milk for whole milk at dinner.

$\begin{array}{lllll}1 & 2 & 3 & 4 & 5\end{array}$

18. Cut down on gravies and cream sauce.

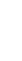

19. Eat poultry and fish instead of red meat at dinner.

$\begin{array}{llll}2 & 3 & 4 & 5\end{array}$

20. Avoid ordering red meat (beef, pork, ham, lamb) at restaurants. 


\section{Sallis' EXERCISE CONFIDENCE SURVEY}

Below is a list of things people might do while trying to increase or continue regular exercise. We are interested in exercises like swimming, brisk walking, bicycle riding, or aerobics classes.

Whether you exercise or not, please rate how confident you are that you could really motivate yourself to do things like these consistently, for at least six months.

Please circle one number for each question. How sure are you that you can do these things?

21. Get up early, even on weekends, to exercise.

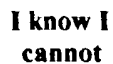

1

22. Stick to your exercise program after a long, tiring day at work.

23. Exercise even though you are feeling depressed.

24. Set aside time for a physical activity program; that is, walking, jogging, swimming, biking, or other continuous activities for at least 30 minutes, 3 times a week.

25. Continue to exercise with others even though they seem too fast or too slow for you.

26. Stick to your exercise program when undergoing a stressful life change (e.g., divorce, death in the family, moving). $\begin{array}{lllllll}1 & 2 & 3 & 4 & 5\end{array}$

27. Attend a party only after exercising.

28. Stick to your exercise program when your family is demanding more time from you.

29. Stick to your exercise program when you have household chores to attend to.

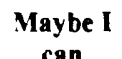

can

I know I

can

$\begin{array}{llll}2 & 3 & 4 & 5\end{array}$

$\begin{array}{lllll}1 & 2 & 3 & 4 & 5\end{array}$

$\begin{array}{lllll}1 & 2 & 3 & 4 & 5\end{array}$

$\begin{array}{lllll}1 & 2 & 3 & 4 & 5\end{array}$

$\begin{array}{lllll}1 & 2 & 3 & 4 & 5\end{array}$

$\begin{array}{lllll}1 & 2 & 3 & 4 & 5\end{array}$


30. Stick to your exercise program even when you have excessive demands at work.

3

45

31. Stick to your exercise program when social obligations are very time consuming.

2

2

$\begin{array}{lll}3 & 4 & 5\end{array}$

32. Read or study less in order to exercise more. 1

2

3

45




\section{Sallis' SOCIAL SUPPORT AND EATING HABITS SURVEY}

\section{Part I}

ID\#

Below is a list of things people might do or say to someone who is trying to improve their eating habits. We are interested in high fat and high salt (high sodium) foods. If you are not trying to make any of these dietary changes, then some of the questions may not apply to you, but please read and give an answer to every question.

Please rate each question twice. Under family, rate how often anyone living in your household has said or done what is described during the last three months. Under friends, rate how often your friends, acquaintances, or coworkers have said or done what is described during the last three months.

Please write one number from the following rating scale in each space.

\section{SAMPLE:}

A. If my family rarely makes fun of the foods I eat, and

Family

Friends my friends very often do, I would answer like this:

A. Made fun of foods I eat

A. 2 A. 5

\begin{tabular}{ccccc} 
none & rarely & $\begin{array}{l}\text { A } \\
\text { few } \\
\text { times }\end{array}$ & often & $\begin{array}{l}\text { very } \\
\text { often }\end{array}$ \\
\hline 1 & 2 & 3 & 4 & 5
\end{tabular}

During the past three months, my family (or members of my household) or friends:

1. Encouraged me not to eat "unhealthy foods"

Friends $\quad \underline{\text { Family }}$ (cake, salted chips) when I'm tempted to do so.

1.

1.

2. Discussed my eating habit, changes with me (asked me how I'm doing with my eating changes). 2.

2.

3. Remind me not to eat high fat, high salt foods.

3.

3.

4. Complimented me on changing my eating habits ("Keep it up", "We are proud of you").

4.

4. 
5. Commented if I went back to my old eating habits. 5 .

5.

6. Ate high fat or high salt foods in front of me.

6.

6.

7. Refused to eat the same foods I eat.

7.

-7 .

8. Brought home foods I'm trying not to eat.

8.

8.

9. Got angry when I encouraged them to eat low salt, low fat foods.

9.

9.

10. Offered me food I'm trying not to eat.

10.

10.

\section{Part II}

\section{Sallis' SOCIAL SUPPORT AND EXERCISE SURVEY}

Below is a list of things people might do or say to someone who is trying to exercise regularly. If you are not trying to exercise, then some of the questions may not apply to you, but please read and give an answer to every question.

Please rate each question twice. Under family, rate how often anyone living in your household has said or done what is described during the last three months. Under friends, rate how often your friends, acquaintances, or coworkers have said or done what is described during the last three months.

Please write one number from the following rating scale in each space:

\begin{tabular}{|c|c|c|c|c|c|}
\hline none & rarely & $\begin{array}{l}\text { A } \\
\text { few } \\
\text { times }\end{array}$ & \multicolumn{2}{|l|}{ often } & $\begin{array}{l}\text { very } \\
\text { often }\end{array}$ \\
\hline \multirow[t]{4}{*}{1} & 2 & 3 & 4 & & 5 \\
\hline & & & & Friends & Family \\
\hline & \multicolumn{2}{|c|}{ 11. Exercised with me. } & & 11. & 11. \\
\hline & \multicolumn{2}{|c|}{ 12. Offered to exercise with me. } & & & \\
\hline
\end{tabular}


13. Gave me helpful reminders to exercise

13.

13. ("Are you going to exercise tonight?")

$\underline{\text { Friends }}$

$\underline{\text { Family }}$

14. Gave me encouragement to stick with my exercise program.

14.

14.

15. Changed their schedule so we could exercise together.

15.

16.

6.

16. Discussed exercise with me.

17. Complained about the time I spend exercising.

17.

17.

18. Criticized me, or made fun of me for exercising.

18.

8.

18.

19. Gave me rewards for exercising (bought me something or gave me something I like.)

19.

19.

20. Planned for exercise on recreational outings.

20.

21.

.

22.

22. Asked me ideas on how they can get more exercise.

23. Talked about how much they like to exercise.
23.

23. 


\section{Appendix D}

Flyers

Awareness Flyer

Recruitment Flyer (TDI)

Recruitment Flyer (CBI)

Poster Contest

Follow-Up Flyer 

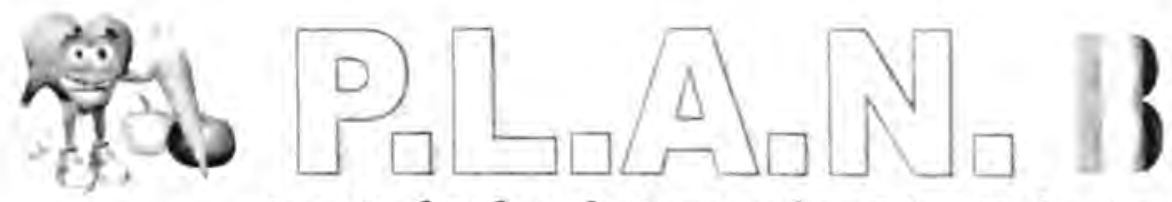

Practical Lifestyle Approach to $\mathcal{N}$ ew Behaviors

\section{Health Education Program Coming Soon}

\section{Begins August 2004.}

\section{All students are invited to participate}

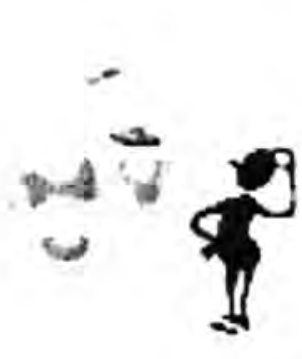

Sessions will include: $\checkmark$ Teen Nutrition

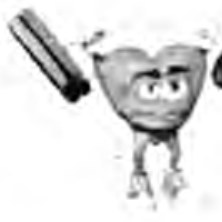
$\checkmark$ Physical Activity

$\checkmark$ Overview of the Nutrients

$\checkmark$ Nutrition and Chronic Disease

$\checkmark$ Taking Responsibility for Your

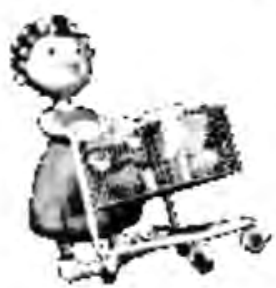

Health

Contact Krista at p.1.a.n.b@comcast.net for further information 

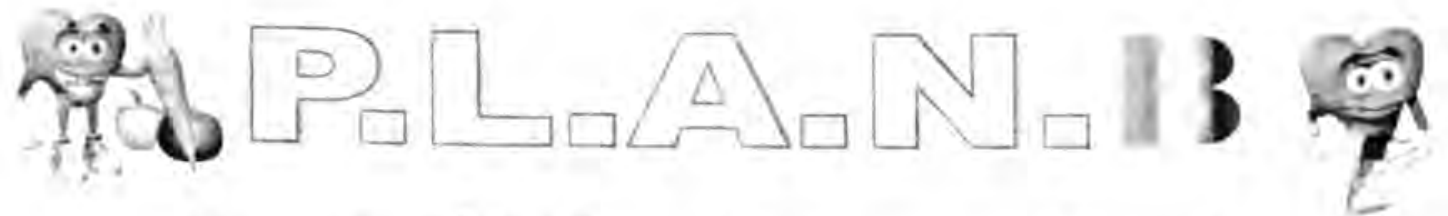

Practical Lifestyle Approach to New Behaviors

Do you want to be a part of a research study that aims to improve the dietary and physical activity habits of high school students?
- Requires meeting 11 times for 45 minutes during your elective courses over a six-month period.

If you are interested, please see Krista at recruitment desk outside the cafeteria pick up parental permission form and class schedule form in room 48 .

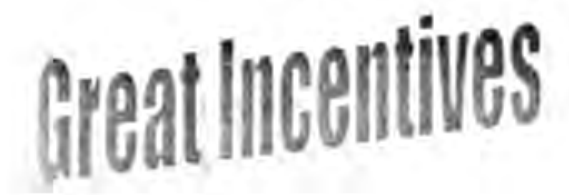

$\checkmark$ T-Shirt

$\checkmark$ Radio

$\checkmark$ Pedometer

$\checkmark$ Samples/Coupons

$\checkmark$ Poster Contest w/ cash prizes

$\checkmark$ Diet analysis

$\checkmark$ School supplies

$\checkmark$.... and more!
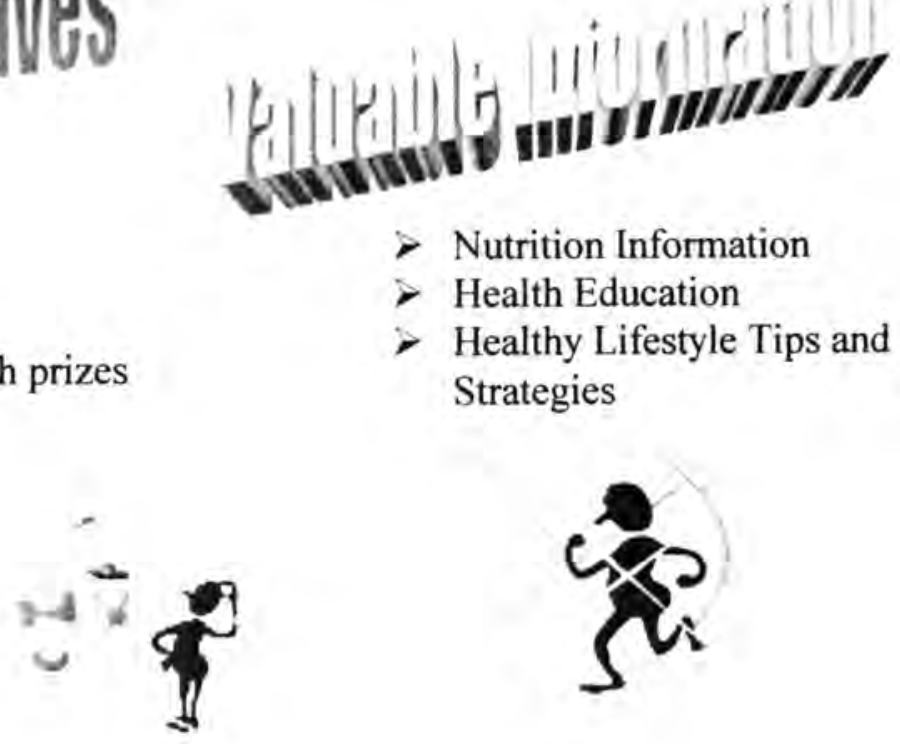

$>$ Nutrition Information

$>$ Health Education

> Healthy Lifestyle Tips and Strategies 

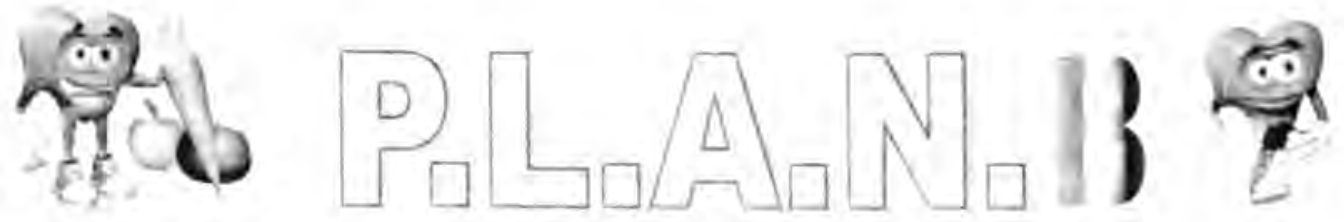

Practical Lifestyle Approach to $\mathcal{N}$ ew Befaviors

Do you want to be a part of a research study that aims to improve the dietary and physical activity habits of teens?

- Requires meeting 11 times for 45 minutes during your elective courses over a six-month period.

If you are interested, please see Krista at recruitment desk outside the cafeteria or email p.1.a.n.b@comcast.net

\section{Great Incentives}

$\checkmark$ T-Shirt

$\checkmark$ Radio

$\checkmark$ Pedometer

$\checkmark$ Samples/Coupons

$\checkmark$ Poster Contest w/ cash prizes

$\checkmark$ Diet analysis

$\checkmark$ School supplies

$\checkmark$....and more!
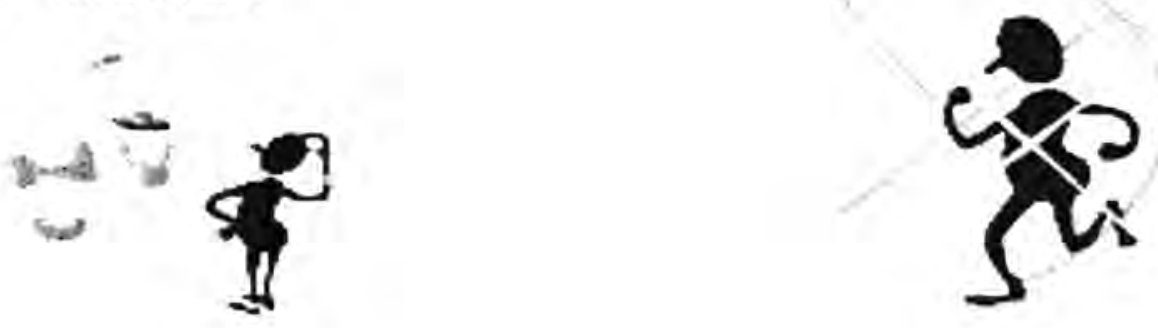


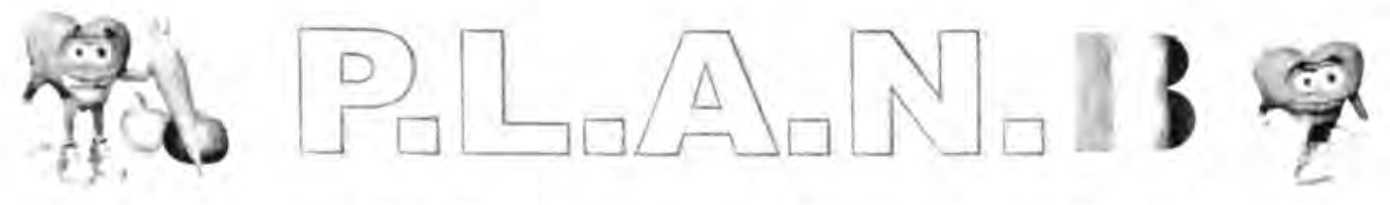

Practical Lifestyle Approach to $\mathcal{N e w}$ Behaviors

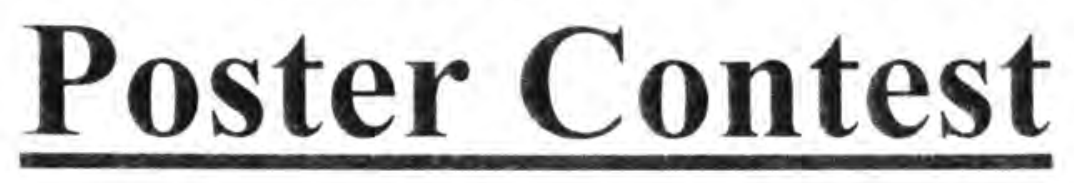

\section{All Posters Should Be Turned in By: \\ December $15^{\text {th }}, 2004$}

Posters will be judged on:

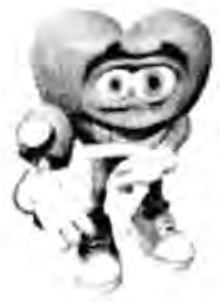

$\checkmark$ Creativity

$\checkmark$ Accuracy

$\checkmark$ Content

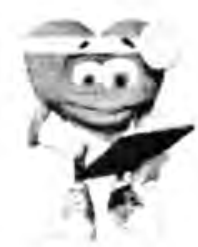

Overall Appearance

Posters can be no larger than 24" X 36 "

\section{CASH Prizes}

First Prize: $\$ 100.00$

Second Prize: $\$ 50.00$

Third Prize: $\$ 25.00$ 


\section{Ad Plan B Follow-Up}

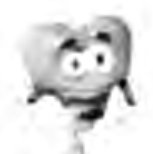

Follow Up Sessions for $\begin{gathered}\text { The ford dive } \\ \text { pryarid }\end{gathered}$

those who participated

in PLAN B will take

place on

April -, 2005 and April —, 2005 in the

Cafeteria. Please ask your teacher to check the list for participant

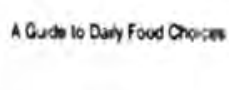

IIt Filsen sum ot ant D bes exd,

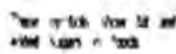

10 2is seres
use soumal

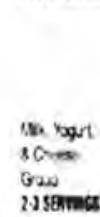

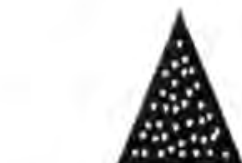

$\because$

- - - - -

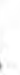

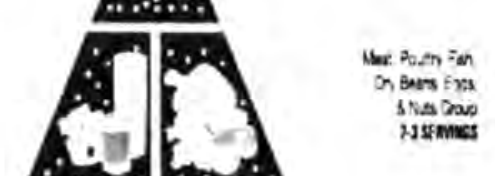

and

SNa Cow
2usfmess

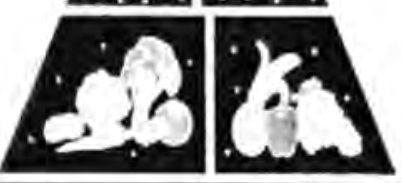

ins

24 tetwer

names and come at the INCENTIVES:

specified time. - On April , 2005

breakfast will be served to every participant!

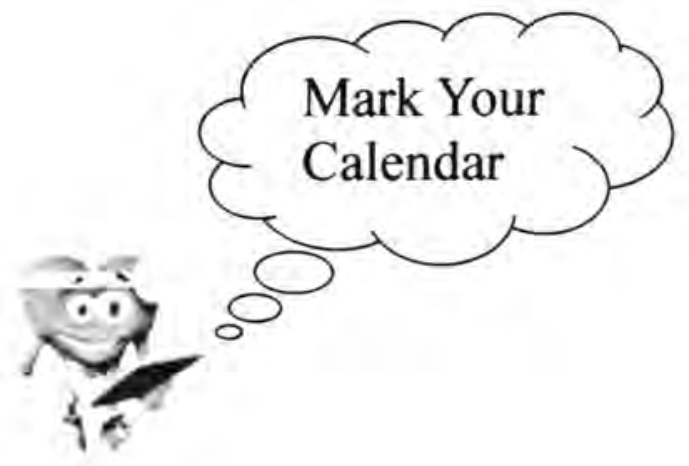

- On April - -

2005 FREE $t$-shirts will

be given to every

participant! 


\section{Appendix E}

Forms Granting Permission to Conduct Research

Florida International University Institutional Review Board

School Board of Broward County

Principals 


\section{MEMORANDUM}

To:

Krista Casazza

CC:

Michelle Ciccazzo

File

From:

Date:

Chris Grayson, Coordinator Institutional Review Board

Proposal Title: June 4, 2004

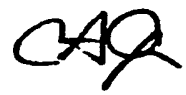

Improving the Dietary and Physical Activity Patterns of Adolescents in South Florida. New Title: Practical Lifestyle Approach to New Behaviors (PLAN B)

Approval \# 121503-01

The Institutional Review Board has approved the following modification(s):

$>$ Elimination of biochemical analysis of participants in order to obtain hemoglobin Alc level and lipid profile.

$>$ An additional component has been added to replace the biochemical analysis.

$>$ Addition of a $5^{\text {th }}$ session to both intervention groups

$>$ Two additional questionnaires added: social support and eating/exercise

. There are no additional requirements in regards to your study. However, if there are changes in the protocol after you commence your study that may increase the risks that the human subjects are exposed to, you are required to resubmit your proposal to a Representative of the IRB for review. Your annual review date is December 2004. If you have any questions please contact your Representative or the IRB Coordinator at 348-2494 or by email at irbiacuc@fiu.edu. 


\section{THE SCHOOL BOARD OF BROWARD COUNTY, FLORIDA RESEARCH SERVICES}

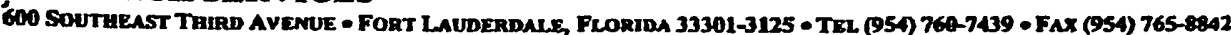

DR. Cary SuTton

Director

sumoncan@hotmail.com

\section{SCHOOL BOARD}

Choir CAROLEL ANDNEWS

Tice Chair STEPHANIE ARMA KRATT. ESO JUDIE S DUDHICK DARLA L CARTER

April 28, 2004
BEVERLY A GALLAGHER

DR ROBERT D. PARKS

MARTY RUBENSTEOY

LOIS WEXKER

remices Deaver

Vermices Inew

DR. FRANK TILL

Superinterdent of Sctiools

Dear Ms. Casazza:

Thank you for submitting your proposal Practical Lifestyle Approach to New Behavions, for consideration by Broward County Public Schools (BCPS). Staff has reviewed your research project and approval has been granted.

This approval means that we have found your proposed rescarch methods to be compatible with a public school setting, and your rescarch questions of interest to the school district. Based on the information you have supplied, your approval to conduct research will expire on August 30, 2005. If you are unable to complete your research by the date indicated, you must contact the Research Services Department in writing and request an extension.

Participation in research, however, is a decision to be reached by the affected principals on a strictly voluntary basis. To assist principals in their decision, please outline the operational steps to be performed by staff at their schools. You are asked to share this information at the same time you provide principals with the attached memorandum. The Approval Memorandum inchudes the Area Superintendent's signature and must be provided to cach principal of each selected research site. These principals will not cooperate with you uniess the Approval Memorandam is provided to them by you.

Based upon the research methods described in your proposal, campus visitations will be required. As a consequence, you will need to follow the fingerprint/background procedures that are required for all researchers and all members of the research team who will be visiting the school campuses. All members of the research tcam must report to our Security Clearance Department at 3530 S. University Drive, Davie Florida, for the fingerprintbackground procedure. A $\$ 60.00$ money order made payable to Fingerprinting Services LLC will be required for each researcher as well as a $\$ 10.00$ money order made payable to the School Board of Broward County for each identification badge. Please call 954-262-5515 to make an appointment. Each rescarcher must bring the completed Security Backgropnd Check Form and a copy of this approval letter to the Security Clearance. Department to initiate the procedure. Please contact Deidre Douglas at 954-760-7349 for the Security Background Check Form.

Per your proposal, the anticipated date for submitting an abstract of the research findings is December 15, 2005. Following the completion of your work, please contact me in writing regarding your research findings. If additional assistance is needed from our staff, please contact me at (954) 712-1595.

Thank you for your request.

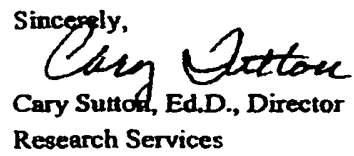

CS:dd

Enclosures 


\section{CARY SUTTON, Ed.D., DIRECTOR \\ DEPARTMENT OF RESEARCH SERVICES}

April 23, 2004

TO: $\quad$ Principals

FROM: $\quad$ Cary Sutton, Ed.D., Director 4

VIA: Verda Farrow, Edo. Superiphendent

SUBJECT: APPROVAL MEMORANDUM FOR RESEARCH PROPOSAL PRACTICAL LIFESTYLE APPROACH TO NEW BEHAVIORS

Staff has reviewed the research proposal Practical Lifestyle Approach to New Behaviors and approval has been granted for the researchers or research group to contact you requesting participation. The recently completed review of the proposed research involved school- and district-based staff, Institutional Review Board (IRB) approvals, and a review of the proposed research methods. These steps were taken to determine if the proposed methods demonstrated reasonable promise of generating data/analyses that will accurately answer the main research questions of interest.

Your participation in this research project is voluntary. To aid your decision, the researcher or research organization has been instructed to share with each selected principal a complete description of research activities as well as this memorandum. Based upon this information, each principal would then be asked to make a decision to participate or not and inform the requesting research parties of the decision.

VF/COS:dd 
MCARTHUR HIGH SCHOOL

Carol Roland, Principal

6501 Hollywood Blvd.

Hollywood, FL 33024

(954)-985-3!50

(954)-985-3154 FAX

May 03, 2004 Vice Chair

CAROLEL ANDREWS STEPIANIE ARMA KRAFT. ESO. JUDIE $S$. DUDNICK DARLA L CARTER BeVERLY A. Gallagher ROBERT D. PARKS. Ed.D. MARTY RUBINSTEIN LOIS WEXLER LOIS WEXLER

DR. FRANX TLL DR. FRANK TILL

Ms. Krista Casazza

11200 S.W. $8^{\text {th }}$ Street

HLS450

Miami, FL 33199

Dear Ms. Casazza:

This letter will serve as an official document indicating that your request to utilize McArthur High School as part of your research project for your dissertation is hereby approved. Please coordinate any and all activities with me in advance so I may facilitate each of your requests in a timely manner.

If I cah ge of any further assistance, please feel free to contact me at anytime.

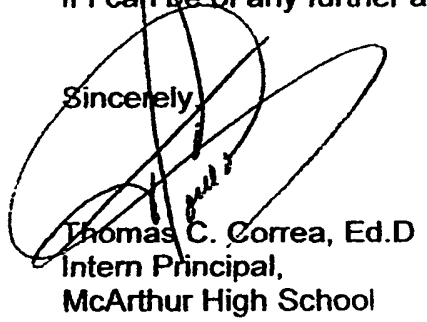




\section{THE SCHOOL BOARD OF BROWARD COUNTY, FLORIDA}

HALANDALE HIGH

Carthoo in Campben, Frincipal

T20 Northwest go Arenase

Itslladale Besch, Florida 33009

954-157-2600

954-457-2629 FAX

SCHOOL, BOARD

May 21, 2004

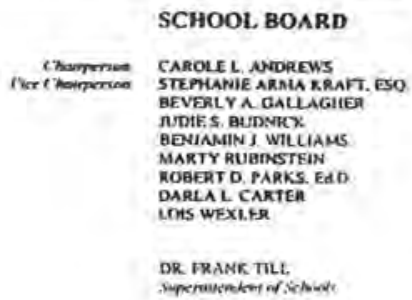

Ms. Krista Casazza

11200 S.W. $8^{\text {th }}$ Street

HLS450

Miami, FL 33199

Dear Ms. Casazza:

This letter will serve as an official document indicating that your request to utilize Hallandale High School as part of your research project for your dissertation is hereby approved.

Please coordinate any and all activities with me and Mrs. Spring in advance so I may facilitate each of your requests in a timely manner.

If I can be of any further assistance, please feel free to contact me at anytime.

Sincerely.

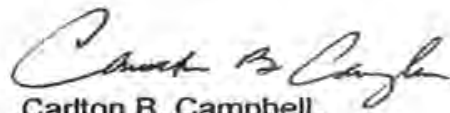

Carlton B. Campbell,

Principal

CBC:CW 
Deerffeld Beach Hilgh School Mis. Kathleen Marilnez, Prtucips! 910 Souihwest is Street Deerfield Beach, Fi 3344I (754) 322-0650 Telephone (75A) 322-07Bo Fectirail?

May 27, 2004
SCHOOL BOARD

Oumporion CAROLEL ANDREWS nee Chatopersein STEPHANIE ARMA KRATT, ESO TUDIE S. DUDNICK. DARIALL CARTER HeVERT MarT riments LOts weXuer

BENIAMRA L. WTLLLAMS

Veraica Prard Ery Blache Hift Schood Sondone Adwion

DR. FRANK TIL Samarintendent of Sracods

Ms. Krista Casazza

11200 S.W. $8^{\text {th }}$ Street HLS450

Miami, FI 33199

Dear Ms. Casazza:

This letter will serve as an official document indicating that your request to utilize Deerfield Beach High School as part of your research project for your dissertation is hereby approved.

Please coordinate any and all activities with me and Mr. Hofstetter in advance so I may facilitate each of your requests in a timely manner.

If I can be of any further assistance, please feel free to contact me at anytime.

Sincerely,

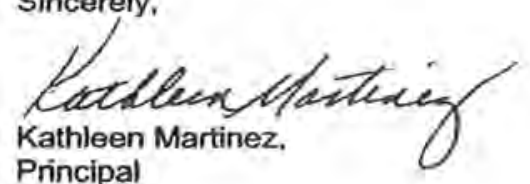

Principal

KM;sc 


\section{Appendix F}

Consent Forms

Parental Consent - English

Parental Consent - Spanish

Parental Consent - Creole

Participant Informed Consent 


\section{PARENTAL CONSENT FOR CHILD PARTICIPATE IN A RESEARCH STUDY}

Title: Practical Lifestyle Approach to New Behaviors (PLAN B): An Innovative Intervention Aimed at Improving the Dietary and Physical Activity Patterns of Adolescents in South Florida

NAME:

Child's Name

Your child has been asked to be in a research study. About 250 other high school students in South Florida will also be invited to take part. Your child's participation will require meeting 10-12 times over the next $\mathbf{8}$ months. We are looking at methods that may improve the eating habits and participation in physical activity by teenagers in South Florida. Before you agree to allow your child to take part, please read this carefully. Be sure that you understand everything and what it means.

Your child will be asked to complete the following at the beginning and end of the study:

1) Nutrition Knowledge questionnaire - to measure your child's knowledge of food and healthy eating

2) Physical activity questionnaire - to find out the average amount of activity your child participates in during a week

3) Measurement of weight and height

4) 24-hour Dietary Recall- your child will be interviewed and asked to remernber all foods and drinks consumed during the past 24 hours

5) Self-efficacy Questionnaire- to find out your child's confidence in their ability to make behavior changes in their eating habits and physical activity habits

6) Social support Questionnaire-to find out the type of support system your child feels he/she has that may impact their ability to make behavior changes in eating and physical activity habits

\section{Expected Benefits}

By being in the study, your child will help us find a useful method for teaching healthy eating and exercise habits. Classes may be designed to include this information and help others teens improve their eating and physical activity pattems, as well.

\section{Possible Risks}

The risks to your child for participating in this study are few. Your child may feel slighlly uncomfortable answering questions about eating and physical activity habits.

\section{Right to Refuse or Withdraw}

Participation is purely by you and your child's own choice. You and your child have the right to decide not to take part in this study. Your child also has the right to withdraw from the study at any time. Taking part is not required by the school and will not affect your child's grades.

\section{Confidentiality}

$\checkmark$ We have taken steps to protect you and your child's privacy.

$\checkmark$ Information completed by your child will not be identifiable as theirs.

$\checkmark$ No names or information that could identify your child individually will be released or made public in any presentation or reports coming from this project.

$$
\begin{aligned}
& \text { College of Health and Urban Affairs } \\
& \text { School of Health }
\end{aligned}
$$

t1200 S.W. 8th Street, HLS 450 * Miami, FL 33199 * Td: (305) 348-2878 * F2s: (305) 348-1996 * hrtp://dietctics-nutrition.fiu.edu

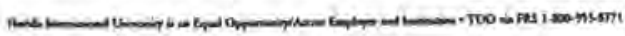




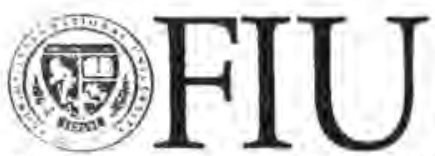

FLORIDA INTERNATIONAL UNIVERSITY

Miami's public research university

Due to privacy concems, information will be given only to you and/or your child.

If you would like to know more about this study, you can contact Krista Casazza or Dr. Michele Ciccazzo at (305) 348-2878. If you feet your child was mistreated or you have questions about your child being in this study you may contact Dr. Bemard Gerstman, the Chairperson of the FIU Institutional Review Board at (305) $348-3115$ or (305) $348-2494$.

\section{Granting Permission}

My signature below shows that I have read the consent form, understand the procedures required for the study, and volumtarily agree to allow my child to participate in the study. I have had a chance to ask questions to heip me understand what my child taking part in this study involves. I am aware of the risks which might be involved with my child's participation and understand that these measures are performed for informational, screening, and/or research purposes only and the principal investigator makes no claims regarding medical diagnoses and/or treatment as a result of information gathered. I agree to allow my child to take part in the study as described and understand that my child may voluntarily withdraw from this study at any time with no adverse impact on his or her grades or participation in academic and/or non-academic programs. Additionally, I have received a copy of this consent form.

Refusing Permission

I have read and understand the above information. However, I do not wish for my child to participate at this time.

\section{Researcher's Affidavit:}

I have carefully explained the nature of the above project to the parent of the participant. I hereby certify that. to the best of my knowledge, the person who is signing this consent form understands clearly the nature, demands, benefits, and risks involved in participation. The parent has consented to allow their child to participate under his/her own free will and I have witnessed the signing. A medical problem or language or educational barrier has not precluded this understanding. 


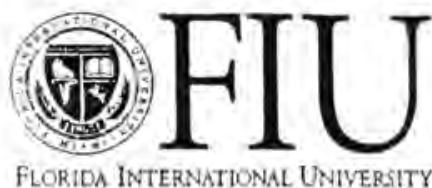

FLORIDA INTERNATIONAL UNIVEREITY

Miamis public research university

\section{CONSENTIMIENTO PATERNO PARA LA PARTICIPACION DE UN MENOR EN UN ESTUDIO INVESTIGATIVO}

Título: Estilo de Vida Práctico Enfocado a Nuevas Conductas (PLAN B): Una intervención innovativa enfocada a mejorar los hábitos dietéticos y la actividad fisica de adolescentes en el sur de la Florida.

NOMBRE:

Nombre del Menor

A su hijo(a) se le ha pedido participe en un estudio investigativo. Alrededor de otros 250 estudiantes de la enseñanza superior en el sur de la Florida serán también invitados a participar. La participación de su hijo(a) requerirá de 10-12 reuniones durante los proximos 6 meses. Nosotros estamos buscando métodos que puedan mejorar los hábitos de alimentación y la participación en actividades fisicas de los adolescentes en el sur de la Florida. Antes de que usted consienta permitir que su hijo(a) participe, por favor lea esto cuidadosamente Esté seguro de que usted comprende todo y lo que significa.

A su hijo(a) se le pedirá completar lo siguiente al comienzo y al final del estudio

1) Cuestionario sobre conocimientos en nutrición - para medir el conocimiento de su hijo(a) sobre alimentos y alimentación sana

2) Cuestionario sobre actividad fisica - para hallar la cantidad promedio de actividad física en la que su hijo(a) participa durante una semana

3) Medida de peso y talla

4) Recordatorio dietéfico de 24 horas - su hijo (a) será entrevistado y se le pedirá recordar todos los alimentos y bebidas consumidos durante las últimas 24 horas

5) Cuestionario sobre auto-eficacia - para hallar la capacidad de su hijo y su habilidad para hacer cambios de comportamiento en sus hábitos de comer y de actividad fisica

6) Cuestionario sobre apoyo social - para hallar que tipo de sistema de apoyo su hijo(a) siente tener que podría impactar su habilidad para realizar cambios de comportamiento en sus hábitos de alimentación y de actividad fisica

7) Cuestionario sobre frecuencia de alimentos - para saber qué tipo de alimentos su hijo(a) ha comido en la última semana.

8) Custionario sobre disponibilidad - para saber cuan listo está su hijo(a) para hacer cambios en sus hàbitos diététicos y de actividad fisica.

\section{Beneficios Esperados}

Por participar en el estudio, su hijo(a) nos ayudará a encontrar un método útil para enseñar hábitos de alimentación sana y de ejercicio. La información obtenida podría ser utilizada en el diseño de clases que ayuden a otros adolescentes a mejorar sus hábitos de alimentación y de actividad fisica.

\section{Posibles Riesgos}

Los riesgos que correrá su hijo(a) por la participación en este estudio son minimos. Su hijo(a) podria sentirse ligeramente incómodo respondiendo preguntas sobre hábitos de comer y de actividad física.

\section{Derecho a Rehusar o a Retirarse}

La participación es puramente una opción suya y de su hijo(a). Usted y su hijo(a) tienen el derecho a decidir el no tomar parte en este estudio. Su hijo(a) también tiene el derecho a retirarse del estudio en cualquier momento. La escuela no requiere la participación y no afectará las notas de su hijo(a).

College of Health and Urban Affairs School of Health

Department of Dieterics and Nutrition

University Park, HLS 450 - Miami, FL 33199. Tel: (305) 348-2878 - Fax: (305) 348.1996

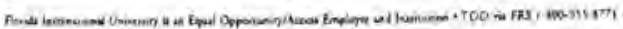




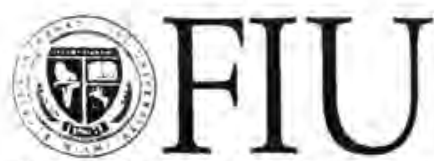

FLORIDA INTERNATIONAL UNIVERSITY

Miami's public research wniversity

\section{Confidencialidad}

$\checkmark$ Nosotros hemos tomado medidas para proteger su privacidad y la de su hijo(a).

$\checkmark$ La información completada por su hijo(a) no será identificable como suya.

$\checkmark$ Ningún nombre o información que pueda identificar a su hijo(a) individualmente será revelada o hecha pública en ninguna presentación o reporte procedente de este proyecto.

$\checkmark$ Por razones de privacidad, información será solamente dada a usted o a su hijo(a).

Si a usted le gustarla saber más sobre este estudio, puede contactar a Krista Casazza o a la Dra. Michele Ciccazzo al (305) 348-2878. Si usted siente que su hijo(a) no fue tratado apropiadamente o tiene preguntas sobre su participación en el estudio, usted puede contactar al Dr. Bernard Gerstman, presidente del Consejo Institucional de Revisión (Institutional Review Board) en la Universidad Internacional de la Florida, al (305) 348 - 3115 o al (305) $348-2494$.

\section{Permiso del Otorgante}

Mi firma debajo significa que yo he leido la planilla de consentimiento, he entendido los procedimientos requeridos por el estudio, y he consentido voluntariamente permitir a mi hijo(a) participar en el estudio. He tenido la oportunidad de hacer preguntas que me ayuden a entender lo que envuelve la participación de mi hijo(a) en este estudio. Soy conciente de los riesgos que podrian estar asociados con la participación de mi hijo(a) y comprendo que estas medidas están solamente desempeñadas con propósitos de información, estudio e investigación; y que el investigador principal no hace declaraciones concernientes a diagnósticos médicos o tratamientos como resultado de la información recogida. Yo doy mi consentimiento para que mi hijo(a) participe en el estudio como se ha descrito, y entiendo que mi hijo(a) puede voluntariamente retirarse de este estudio en cualquier momento sin ningún impacto adverso en sus notas o en su participación en programas académicos y no académicos. Adicionalmente, yo he recibido una copia de esta planilla de consentimiento.

Firma del Padre/Madre

Fecha

\section{Permiso de Negación}

Yo he leido y comprendido la información anterior. Sin embargo, yo no deseo que mi hijo(a) participe en este momento.

Firma del Padre/Madre

Fecha

\section{Affidávit del Investigador:}

Yo he explicado cuidadosamente la naturaleza del proyecto anterior al padre/madre del participante. Por la presente certifico que, según mi leal entender, la persona quien está firmando esta planilla de consentimiento entiende claramente la naturaleza, demandas, beneficios y riesgos envueltos en la participación. El padre/madre ha consentido el permitir a su hijo(a) participar bajo su propia y libre voluntad, y yo he presenciado su firma. Ningún problema médico, de lenguaje, o barrera educacional ha impedido este entendimiento. 


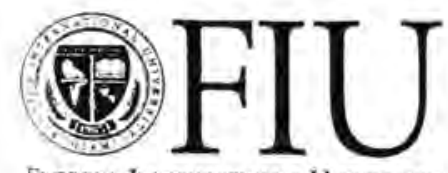

FLORIDA INTERNATIONAL UNIVERSITY

Miami's public research university

\title{
PAPYE KONSANTI POU PARAN TIMOUN KAP PATISIPE NAN YON ETID
}

\author{
Tit: Yon Inovasyon Entèvansyon ki Vle Amelyore Kijan Jen Timoun Nan \\ Florid la Manje ak fê Ekzèsis
}

Non:

Non Timoun

Yo mande timoun ou an pou li patisipe nan etid. Ap gen prèske 250 lot elèv ki nan high school nan Florid la kap patisipe. Paske pitit ou a patisipe nan etid sa nap mande pou nou wè'l 10-12 fwa nan 6 mwa. Nap gade ak kijan nou ka amelyore kijan jan yo manje ak patisipasyon nan fe ekzèsis jèn timoun $\mathrm{ki}$ an Floride di sid la. Avan ou dakò pou pitit ou a patisispe nan etid sa li lèt sa byen. Fòk ou konprann tout bagay trè byen e kisa li vle di. Yap mande piti ou a pou li ranpli bagay sa nan Kòmansman ak nan finisman etid la.

1) Yo kesyonè sou Konesans nitrisyon'w: Pou nou mesire sa ou konnen sou manje ak kijan pou'w manje

2) Kesyonè sou ekzèsis - Pou nou wè ki valè ekzèsis piti ou a fê pandan semenn

3) Pran pwa ak otè timoun nan

4) 24-èd tan manje li te manje- Nap mande pitit ou a pou li sonje tout manje li te manje ak tout sa yo te bwè nan jou ki te pase a (24 èd tan)

5) Kesyonè sou tèt ou- Pou nou ka wè si pitit ou a ka fè chanjman nan vi'l sou kijan'l manje ak fè ekzèsis.

6) Kesyonè sou zafè sosyal- Pou nou ka wè ki sa kijan pitit ou a santi'l sou sa ki ka afekte'l chanjman sou kijan ou yo manje ak fè ekzèsis.

7) Kesyonè sou manje (Lè)- Pou nou ka wè ki kalite manje pitit ou a manje nan semen ki sot pase a.

8) Kesyonè sou preparasyon: Pou nou ka wé si pitit ou a pare pou li fee chanjman nan vi'l sou kijan li manje ak fè ekzèsis.

\section{Benefis}

Patisipasyon Pitit ou a nan etid sa ap ede nou develop yon pi bon metòd sou kijan pou moun manje ki bon pou sante ak ekzèsis. Sa ka ede nou föme cou (klas) pou nou montre lòt moun kijan pou yo manje ak ekzèsis pi byen.

\section{Danje}

Pa gen oken danje ki ka rive pitit ou a paske li patisipe nan etid sa. Pitit ou ka santi'l yon $\mathrm{ti}$ jan malalès pou li repon kèk kesyon sou zafé manje ak ekzèsis.

College of Health and Urban Affairs

Robert R. Stempel School of Public Health

Program in Dieterics and Nutrition

11200 S.W 8 th Street, HLS.1 450 - Miami, FL 33199 - Tel: (305) 348-2878 - Fax: (305) 948-1996 


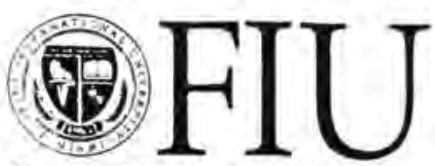

FLORIDA INTERNATIONAL UNIVERSITY

Miami's public research universiry

\section{Dwa pou Pa Partisipe}

Patisipasyon se volonte pitit ou a sa vle di se chaw'l. Li gen dwa pou li pa patisipe nan etid la. Li gen dwa tou pou li soti nan etid ninpót lé li vle. Patisipasyon pitit ou nan etid la pa mele nan zafè lekòl li. Lekòl pa mande'l pou li fê pati etid sa. Patisipasyon '1 pap gen oken efé sou nòt lekòl li

\section{Konfidans (Sekrè)}

$\checkmark$ Nou pran anpil prekosyon pou nou poteje vi prive pitit ou a

$\checkmark$ Infomasyon ke pitit ou a ranpli a ak rete sekrè pa gen moun ka idantifye'l

$\checkmark \mathrm{Pa}$ gen non oubyen infomasyon ki ka idantifye pitit ou a ke yap itilize nan pou nou fe oken aktivite piblik sou pwojè a tout bagay ap rete sekrè

$\checkmark$ Pou nou ka kenbe tout bagay sekrè, se sèl ou ak pitit ou a ke nap bay infomasyon yo.

Si ou bezwen konnen plis infomasyon sou etid sa ou ka kontakte Krista Casazza or Dr. Michele Ciccazzo nan nimewo sa (305) 348-2878. Si ou santi ke yap maltrete pitit ou a oubyen ou gen kesyon paske pitit ou a patisipe nan etid sa ou ka kontakte tout Dr.

Bernard Gerstman, moun ki an tèt FIU Institutional Review Board nan nimewo sa (305) $348-3115$ or (305) $348-2494$.

\section{Pèmisyon (ban pèmisyon)}

Kote mwen siyin anba vle di konsa ke mwen li papye sa e omwen konprann tout bagay trè byen. Mwen fe yon chwa volonté sa vle di ou dakó pou pitit mwen an patisipe nan etid sa. Mwen te gen chans pou mwen mande tout kesyon sou etid la ak patisipasyon pitit mwen an. Mwen konnen ke pa gen oken danje ki ka rive pitit mwen an paske li patisipe nan etid sa. Mwen konprann ke tout infornasyon ke yo pran se pou yo fê rechèch sèlman moun kap fê etid lapa di'm anyen sou afè medikal ak tretman. Mwen dakò pou mwen kite pitit mwen an patisipe nan etid sa, mwen konprann ke li ka soti nan etid la ninpòt lè li vle sa pap gen oken efè sou nòt lekòl li. Mwen resevwa yon kopi nan fom konsanti a.

Siyati Paran

Dat

\section{Pa bay Pèmisyon}

Mwen li e mwen konprann tout infòmasyon sa yo men mwen pa dakò pou pitit mwen an patisipe nan etid sa.

Siyati Paran

Dat

College of Health and Urban Affaits

Rebert R. Stempel School of Public Health

Program in Dieterics and Nutrition

11200 S.W. 8th Streer, HLS-1 450 - Miami, FL 33199 - Tel: (305) 348-2878 - Fax: (305) 348-1996

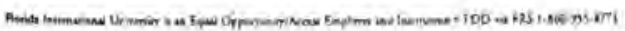




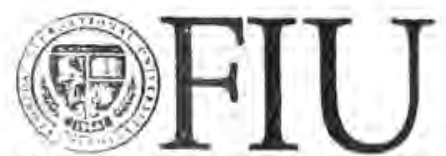

FLORIDA INTERNATIONAL UNIVERSTTY

Miamis public research university

\title{
CONSENT TO PARTICIPATE IN A RESEARCH STUDY
}

\author{
Title: Practical Lifestyle Approach to New Behaviors (PLAN B): An \\ Innovative Intervention Aimed at Improving the Dietary and Physical \\ Activity Patterns of Adolescents in South Florida
}

NAME:

Date of Birth:

Grade:

You are invited to be in a research study. About 250 other high school students in South Florida will also be invited to take part. Your participation will require meeting about 12 times over the next 6 months. We are looking at methods that may improve the eating habits and participation in physical activity by teenagers in South Flonida. Before you agree to take part, please read this carefully. Be sure that you understand everything and what it means.

You will be asked to complete the following at the beginning and end of the study:

1) Nutrition Knowledge questionnaire - to measure your knowledge of food and healthy eating

2) Physical activity questionnaire - 10 find out the average amount of activity you do in a week

3) Measurement of weight and height

4) 24-hour Dietary Recall- you will be interviewed and asked to remember all foods and drinks consumed during the past 24 hours

5) Self-efficacy Questionnaire- to find out your confidence in your ability to make behavior changes in your eating habits and physical activity habits

6) Social support Questionnaire- to find out the type of support systern you have that may impact your abifity to make behavior changes in your eating and physical activity habits

\section{Expected Benefits}

By being in the study, you will help us find a useful method for leaching healthy eating and exercise habits. Classes may be designed to include this information and help other teens improve their eating and physical activity pattems, as well.

\section{Possible Risks}

The risks to you for participating in this study are few. You may feel slightly uncomfortable answering questions about eating, physical activity habits, self-efficacy, and social support.

\section{Right to Refuse or Withdraw}

Participation is purely by your own choice. You have the right to decide not to take part in this study. You also have the right to withdraw from the study at any time. Taking part is not required by the school and will not affect your grades.

\section{Confidentiality}

$\checkmark$ We have taken steps to protect your privacy

$\checkmark$ All responses are kept under a code number, not your name, and will be kept in a locked cabinet in the office of the PI.

$\checkmark$ No names or information that could identify you will be released or made public in any presentation or reports coming from this project.

$\checkmark$ Due to privacy concerns, information will be given only to you and/or your parent.

$$
\begin{aligned}
& \text { College of Health and Urban Affairs } \\
& \text { School of Halth } \\
& \text { Department of Dietetics and Nutrition }
\end{aligned}
$$

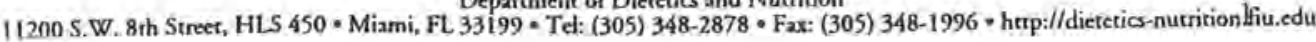

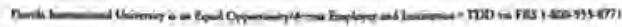




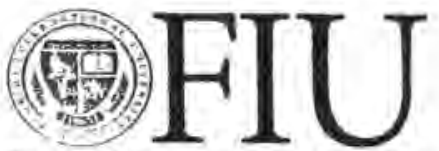

FLORIDA INTERNATIONAL UNIVERSTTY

Miami's public reseanch university

If you or your parent would like to know more about this study, you can contact Krista Casazza or Dr. Michele Ciccazzo at (305) 348-2878. If you feel you were mistreated or have questions about being in this study you may contact Dr. Bemard Gerstman, the Chairperson of the FIU Institutional Review Board at (305) $348-3115$ or (305) 348-2494.

My signature below shows that I have read the consent form, understand what $I$ am being asked to do for the study, and agree to take part in the study. I have had a chance to ask questions to help me understand what taking part in this study involves. I am aware of the risks which might be involved with my taking part and understand that these tests are being done for informational, screening, and/or research purposes only and understand that the principal investigalor makes no claims regarding medical diagnoses and/or treatment as a result of information gathered. I agree to take part in the study as described and understand that I may withdraw from this study at any time without affecting my grades or other school programs. Also. I have received a copy of this consent form.

\section{Researcher's Affidavit:}

I have carefully explained the nature of the above project to the participant. I hereby certify that, to the best of my knowledge, the person who is signing this consent form understands clearly the nature, demands, benefits, and risks involved in participation. The participant has consented under his/her own free will and I have witnessed the signing. A medical problem or language or educational barrier has not precluded this understanding. I have offered him/her a copy of the consent form. 


\section{EDUCATION}

\begin{tabular}{llll} 
Degree & Institution & Field & Dates \\
\cline { 2 - 3 } Ph.D. Doctoral Cand. & FIU & Nutrition & Aug 99-Present \\
Master of Science & University of Florida Animal Nut and Phys & Jan 96-May 97 \\
Bachelor of Science & University of Florida Zoology (minor in Ed.) & Aug 91 - Dec 95
\end{tabular}

FULL-TIME ACADEMIC EXPERIENCE

Institution

Broward County Schools
Rank

Faculty
Field

Science
Dates

Aug 97-02

PART-TIME ACADEMIC EXPERIENCE

Institution

Rank Field

Broward Community College

Palm Beach Community Coll

Adjunct Faculty

Adjunct Faculty
Biological Sciences

Biological Science
Dates

May 99-Pres

Jan 99-May 99

NON-ACADEMIC EXPERIENCE

Place of Employment

Health South Rehab. Hospital

Broward County Schools
Title

Diet Clerk

Substitute Teacher
Dates

Jan 2002 - Mar 2003

Jan 2003-Present

\section{EMPLOYMENT RECORD AT FIU}

Rank

Teaching Assistant/Graduate Assistant

FIU

AHEC Project Coordinator

Dates

FIU

Grant Assistant

Aug 2002 - 2005

Jan 2004-2005

Jan 2004-2005

\section{PUBLICATIONS IN DISCIPLINE}

Casazza $\mathrm{K}$ and Ciccazzo $\mathrm{M}$. Improving the dietary patterns of adolescents using a computer based approach. J Sch Health 2006,76,2, 43-46.

Casazza $\mathbf{K}$ and Ciccazzo M. Method of delivery may have an effect on improving the lifestyle habits of adolescents. Eating Behaviors. [In Press].

George VA, Erb AF, Harris CL, Casazza K. Psychosocial risk factors for eating disorders in Hispanic females of diverse ethnic background and non-Hispanic females. Eating Behaviors [In Press].

Casazza $\mathrm{K}$ and Ciccazzo M. An intervention to improve the dietary and physical activity habits of adolescents in South Florida. (Abstract). JNEB;2005: 37,4, Supplement. 
Casazza K and Ciccazzo M. Practical Lifestyle Approach to New Behaviors (PLANB): An intervention to improve the health of a diverse group of adolescents. (Abstract). $J A D A ; 2005$ : 105,8, Supplement-2, A-11.

\section{WORKS IN PROGRESS}

George VA, Schilit L, Johnson P, Harris CL, and Casazza K. A valid and reliable questionnaire to assess physical activity in university students. Am J Health Beh. (Out for Review).

George VA, Stevenson J, Harris CL, and Casazza K Promotion of Physical Activity by RDs and CDE-RDs to older adults with Type 2 Diabetes. J Nutr Ed Behav (Out for Review).

PRESENTED PAPERS, LECTURES, EXHIBITIONS, AND PERFORMANCES

July 2005 Society for Nutrition Educators Annual Symposium Hyatt Grand Cypress, Orlando, FL

October 2005 ADA Foods \& Nutrition Conference \& Expo America's Center, St. Louis, MO

April 2006 National Priester Health Conference

Hyatt Regency,

Louisville, KY

\section{ORGANIZATION MEMBERSHIPS}

American Dietetic Association (ADA)

Broward County Dietetic Association (BCDA)

Florida Dietetic Association (FDA)

North American Association for the Study of Obesity (NAASO)

American Society for Nutritional Sciences (ASNS)

Association of Graduate Students in Dietetics and Nutrition (AGSDN)

\section{PROFESSIONAL HONORS, PRIZES, FELLOWSHIPS}

Dissertation Year Fellowship, Florida International University, 2005-2006

Florida Dietetic Association Student Stipend, 2005

Florence Bayuk Graduate Fellowship in Health, Florence Bayuk Foundation, 2003-2004

Nominated Teacher of the Year 1999

Elected Science Department Head 1999

\section{NON-PROFIT ORGANIZATIONS}

Florida Introduces Physical Activity and Nutrition to Youth (FLIPANY), Co-Founder 